\title{
Unitary transformations of fibre functors
}

\author{
Dominic Verdon \\ School of Mathematics \\ University of Bristol \\ dominic.verdon@bristol.ac.uk
}

January 13, 2022

\begin{abstract}
We study unitary pseudonatural transformations (UPTs) between fibre functors $\operatorname{Rep}(G) \rightarrow$ Hilb, where $G$ is a compact quantum group. For fibre functors $F_{1}, F_{2}$ we show that the category of UPTs $F_{1} \rightarrow F_{2}$ and modifications is isomorphic to the category of finite-dimensional *-representations of the corresponding bi-Hopf-Galois object. We give a constructive classification of fibre functors accessible by a UPT from the canonical fibre functor, as well as UPTs themselves, in terms of Frobenius algebras in the category $\operatorname{Rep}\left(A_{G}\right)$, where $A_{G}$ is the Hopf *-algebra dual to the compact quantum group. As an example, we show that finite-dimensional quantum isomorphisms from a quantum graph $X$ are UPTs between fibre functors on $\operatorname{Rep}\left(G_{X}\right)$, where $G_{X}$ is the quantum automorphism group of $X$.
\end{abstract}

\section{Introduction}

\subsection{Overview}

Compact symmetry groups play a crucial role in quantum physics. By Tannaka duality, a compact group $G$ is interchangeable with its category of finite dimensional representations $\operatorname{Rep}(G)$, with canonical unitary monoidal fibre functor $F: \operatorname{Rep}(G) \rightarrow$ Hilb, where Hilb is the category of finite-dimensional Hilbert spaces and linear maps. One can therefore equivalently say that categories of representations of compact groups with a fibre functor play a crucial role in quantum physics. One way to interpret such categories is as encoding a consistent structure of system types, fusion rules, permissible transitions, etc. The fibre functor then assigns state spaces to all of the systems in the theory, and can be seen as a representation of this compositional structure as part of finite-dimensional quantum theory. Schematically:

$$
\begin{array}{ccc}
\text { Compositional category } & \rightarrow & \text { Representation } \\
\operatorname{Rep}(G) & \rightarrow & \text { Hilb }
\end{array}
$$

One can generalise this notion of representation to theories more general than those described by the representation category of a compact group. In particular, we can consider representations of $C^{*}$-tensor categories with conjugates. Such categories and their fibre functors are described by the representation theory of compact quantum groups, and their associated Hopf-Galois objects.

In [19] we introduced a notion of unitary pseudonatural transformation relating two monoidal functors, or more generally two pseudofunctors. This paper is a study of unitary pseudonatural transformations between fibre functors on $C^{*}$-tensor categories with conjugates, or equivalently (provided a fibre functor exists) representation categories of compact quantum groups. The physical significance of these transformations will be explained in forthcoming work.

${ }^{0} 2020$ Mathematics Subject Classification. Primary 20G42, 18M40, 81R50; Secondary 18M30. 
Unitary pseudonatural transformations. Unitary pseudonatural transformations are a generalisation of unitary monoidal natural isomorphisms, defined as follows. Let $\mathcal{C}$ be a $C^{*}$-tensor category with conjugates, and let $F, F^{\prime}: \mathcal{C} \rightarrow$ Hilb be fibre functors. Then a unitary pseudonatural transformation specifies:

- A Hilbert space $H$.

- For every object $X$ of $\mathcal{C}$, a unitary linear map $F(X) \otimes H \rightarrow H \otimes F^{\prime}(X)$.

These unitaries must obey equations generalising the monoidality and naturality conditions for unitary monoidal natural isomorphisms $F \rightarrow F^{\prime}$, which are recovered when $H=\mathbb{C}$.

Hopf-Galois theory. Let $\mathcal{C}$ be a $C^{*}$-tensor category with conjugates. Whenever a fibre functor $F: \mathcal{C} \rightarrow$ Hilb exists, we can construct a monoidal equivalence $\mathcal{C} \simeq \operatorname{Rep}(G)$ for a compact quantum group $G$. The category $\mathcal{C}$ can therefore be understood in terms of the compact quantum group $G$, or rather its dual Hopf $*$-algebra $A_{G}$.

Let $F_{1}, F_{2}: \mathcal{C} \rightarrow$ Hilb be fibre functors corresponding to compact quantum groups $G_{1}, G_{2}$. Then one can construct an $A_{G_{2}}-A_{G_{1}}$-bi-Hopf-Galois object $Z$ linking the two fibre functors. This is a $*$-algebra with a compatible left and right coactions of the algebras $A_{G_{2}}, A_{G_{1}}$.

Here we show (Theorem 3.14) that there is an isomorphism of categories between:

- The category $\operatorname{Rep}(Z)$ of finite-dimensional $*$-representations of $Z$ and intertwining linear maps.

- The category $\operatorname{Hom}\left(F_{1}, F_{2}\right)$ of unitary pseudonatural transformations $F_{1} \rightarrow F_{2}$ and modifications.

This generalises the known fact [3, Thm 4.4.1] that the 1-dimensional $*$-representations of an $A_{G_{2}}{ }^{-}$ $A_{G_{1}}$-bi-Hopf-Galois object correspond to unitary monoidal natural transformations $F_{1} \rightarrow F_{2}$.

Morita theory. In [19] we showed that the 2-category $\operatorname{Fun}(\mathcal{C}$, Hilb $)$ of unitary fibre functors, unitary pseudonatural transformations and modifications has certain nice properties; in particular, it is a pivotal dagger 2-category with split dagger idempotents. This makes it an appropriate setting for Morita theory [15, Appendix].

Let us fix some fibre functor $F: \mathcal{C} \rightarrow$ Hilb. By the results just discussed, the endomorphism category $\operatorname{End}(F)$ of UPTs $F \rightarrow F$ and modifications is isomorphic to the category $\operatorname{Rep}\left(A_{G}\right)$ of f.d. *-representations of the associated compact quantum group algebra.

We use Morita theory to classify fibre functors $F^{\prime}$ such that there exists a UPT $F \rightarrow F^{\prime}$, as well as UPTs $F \rightarrow F^{\prime}$, in terms of certain algebraic structures called simple Frobenius monoids in the category $\operatorname{Rep}\left(A_{G}\right)$. In particular, we give constructions setting up a correspondence between the following structures (Theorem 4.14):

- Unitary monoidal isomorphism classes of unitary fibre functors accessible from $F$ by a UPT; and Morita equivalence classes of simple Frobenius monoids in $\operatorname{Rep}\left(A_{G}\right)$.

- Equivalence classes of UPTs $\alpha: F \rightarrow F^{\prime}$ for some accessible fibre functor $F^{\prime}$; and $*$-isomorphism classes of simple Frobenius monoids in $\operatorname{Rep}\left(A_{G}\right)$.

As a consequence, we obtain a concrete construction of fibre functors accessible from $F$ by a UPT in terms of idempotent splitting (Theorem 4.12).

Quantum graph isomorphisms. As an example of UPTs between fibre functors, we show that, for finite quantum graphs $X, Y$, the finite-dimensional quantum graph isomorphisms $X \rightarrow Y$ (Definition 5.11) considered in quantum information theory $[1,5]$ are UPTs between accessible fibre functors on the category $\operatorname{Rep}\left(G_{X}\right)$ of representations of the quantum automorphism group of $X$. This sets up an equivalence between the following 2-categories (Theorem 5.20): 
- QGraph ${ }_{X}$ : Objects - quantum graphs f.d. quantum isomorphic to $X$. 1-morphisms - f.d. quantum isomorphisms. 2-morphisms - intertwiners.

- Fun $\left(\operatorname{Rep}\left(G_{X}\right), \text { Hilb }\right)_{X}$ : Objects - Fibre functors accessible by a UPT from the canonical fibre functor on $\operatorname{Rep}\left(G_{X}\right)$. 1-morphisms - UPTs. 2-morphisms - modifications.

\subsection{Acknowledgements}

The author thanks Julien Bichon, Amaury Freslon, Laura Mančinska, Ashley Montanaro, Benjamin Musto, David Reutter, David Roberson, Changpeng Shao, Jamie Vicary and Makoto Yamashita for useful discussions related to this work. The work was supported by EPSRC.

\section{$1.3 \quad$ Structure}

In Section 2 we introduce necessary mathematical background material for this paper. In Section 3 we discuss the relationship between UPTs and Hopf-Galois theory. In Section 4 we discuss the Morita classification/construction of accessible UPTs and fibre functors. In Section 5 we show that finitedimensional quantum graph isomorphisms are UPTs.

\section{Background}

\subsection{Pivotal dagger categories and their diagrammatic calculus}

\subsubsection{Monoidal categories}

We assume the reader is familiar with the definition of a monoidal category [12]. We use the standard coherence theorem [11] to assume that all our monoidal categories are strict, allowing the use of the following well-known diagrammatic calculus [17].

We read diagrams from bottom to top. Objects are drawn as wires, while morphisms are drawn as boxes whose type corresponds to their input and output wires. Composition of morphisms is represented by vertical juxtaposition, while monoidal product is represented by horizontal juxtaposition. For example, two morphisms $f: X \rightarrow Y$ and $g: Y \rightarrow Z$ can be composed as follows:

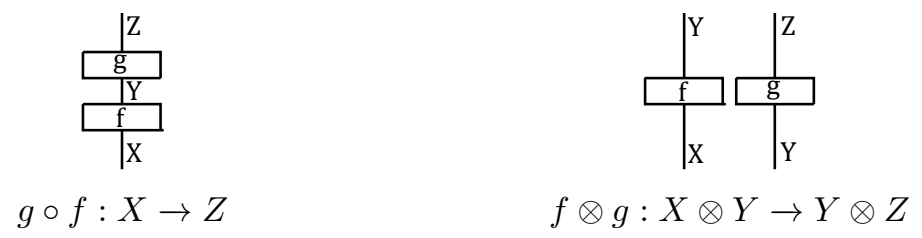

The wire for the monoidal unit $\mathbb{1}$, and the identity morphism $\operatorname{id}_{X}$ for any object $X$, are invisible in the diagram. Two diagrams which are planar isotopic represent the same morphism [17].

\subsubsection{Pivotal categories}

We recall the notion of duality in a monoidal category.

Definition 2.1. Let $X$ be an object in a monoidal category. A right dual $\left[X^{*}, \eta, \epsilon\right]$ for $X$ is:

- An object $X^{*}$.

- Morphisms $\eta: \mathbb{1} \rightarrow X^{*} \otimes X$ and $\epsilon: X \otimes X^{*} \rightarrow \mathbb{1}$ satisfying the following snake equations:
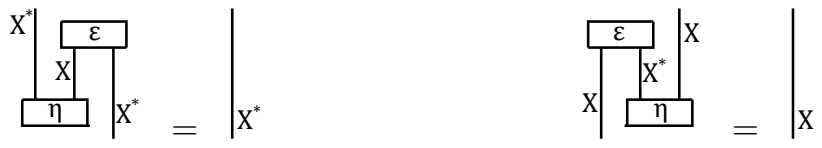
A left dual $\left[{ }^{*} X, \eta, \epsilon\right]$ is defined similarly, with morphisms $\eta: \mathbb{1} \rightarrow X \otimes{ }^{*} X$ and $\epsilon:{ }^{*} X \otimes X \rightarrow \mathbb{1}$ satisfying the mirror images of (5).

We say that a monoidal category $\mathcal{C}$ has right duals (resp. has left duals) if every object $X$ in $\mathcal{C}$ has a chosen right dual $\left[X^{*}, \eta, \epsilon\right]$ (resp. a chosen left dual).

To represent duals in the graphical calculus, we draw an upward-facing arrow on the $X$-wire and a downward-facing arrow on the $X^{*}$ - or ${ }^{*} X$-wire, and write $\eta$ and $\epsilon$ as a cup and a cap, respectively. Then the equations (5) become purely topological:

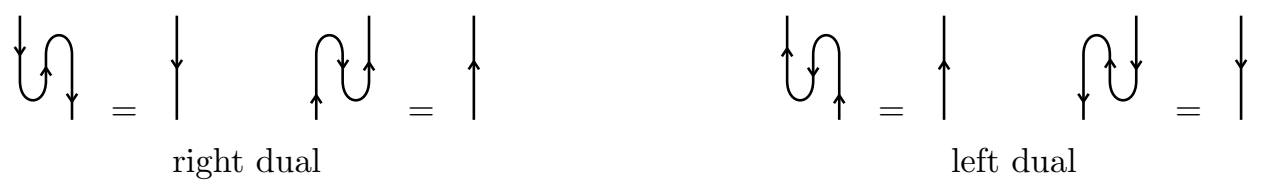

Proposition 2.2 ([8, Lemmas 3.6, 3.7]). If $\left[X^{*}, \eta_{X}, \epsilon_{X}\right]$ and $\left[Y^{*}, \eta_{Y}, \epsilon_{Y}\right]$ are right duals for $X$ and $Y$ respectively, then $\left[Y^{*} \otimes X^{*}, \eta_{X \otimes Y}, \epsilon_{X \otimes Y}\right]$ is right dual to $X \otimes Y$, where $\eta_{X \otimes Y}$ and $\epsilon_{X \otimes Y}$ are defined by:

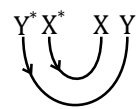

$\eta X \otimes Y$

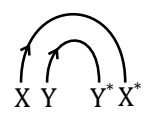

$\epsilon_{X \otimes Y}$

Moreover, $\left[\mathbb{1}, \mathrm{id}_{\mathbb{1}}, \mathrm{id}_{\mathbb{1}}\right]$ is right dual to $\mathbb{1}$. Analogous statements hold for left duals.

Duals are unique up to isomorphism.

Proposition 2.3 ([8, Lemma 3.4$])$. Let $X$ be an object of a monoidal category, and let $\left[X^{*}, \eta, \epsilon\right],\left[X^{* \prime}, \eta^{\prime}, \epsilon^{\prime}\right]$ be right duals. Then there is a unique isomorphism $\alpha: X^{*} \rightarrow X^{* \prime}$ such that
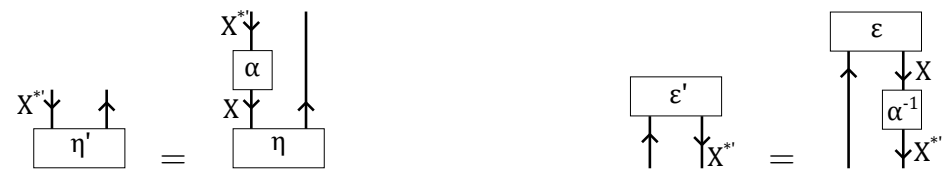

An analogous statement holds for left duals.

In a category with duals, we can define a notion of transposition for morphisms.

Definition 2.4. Let $X, Y$ be objects with chosen right duals $\left[X^{*}, \eta_{X}, \epsilon_{X}\right]$ and $\left[Y^{*}, \eta_{Y}, \epsilon_{Y}\right]$. For any morphism $f: X \rightarrow Y$, we define its right transpose $f^{T}: Y^{*} \rightarrow X^{*}$ as follows:

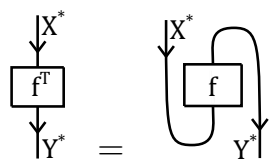

For left duals ${ }^{*} X,{ }^{*} Y$, a left transpose may be defined analogously.

In this work we are mostly interested in categories with compatible left and right duals. Such categories are called pivotal.

Let $\mathcal{C}$ be a monoidal category with right duals. It is straightforward to check that the following defines a monoidal functor $\mathcal{C} \rightarrow \mathcal{C}$, which we call the double duals functor:

- Objects $X$ are taken to the double dual $X^{* *}:=\left(X^{*}\right)^{*}$. 
- Morphisms $f: X \rightarrow Y$ are taken to the double transpose $f^{T T}:=\left(f^{T}\right)^{T}$.

- The monoidal structure is defined using the isomorphisms of Proposition 2.3.

Definition 2.5. We say that a monoidal category $\mathcal{C}$ with right duals is pivotal if the double duals functor is monoidally naturally isomorphic to the identity functor.

Roughly, the existence of a monoidal natural isomorphism in Definition 2.5 comes down to the following statement:

- For every object $X: r \rightarrow s$, there is an isomorphism $\iota_{X}: X^{* *} \rightarrow X$.

- These $\left\{\iota_{X}\right\}$ can be chosen compatibly with composition and monoidal product in $\mathcal{C}$.

In a pivotal category, for any object $X$ the right dual $X^{*}$ is also a left dual for $X$ by the following cup and cap (we have drawn a double upwards arrow on the double dual):
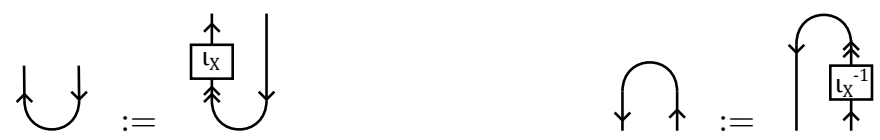

With these left duals, the left transpose of a morphism is equal to the right transpose. Whenever we refer to a pivotal category from now on, we suppose that the left duals are chosen in this way.

There is a very useful graphical calculus for these compatible left and right dualities in a pivotal category. To represent the transpose, we make our morphism boxes asymmetric by tilting the right vertical edge. We now write the transpose by rotating the boxes, as though we had 'yanked' both ends of the wire in the RHS of (8):

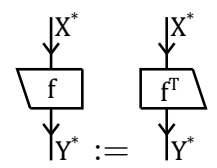

Using this notation, morphisms now freely slide around cups and caps.

Proposition 2.6 ([8, Lemma 3.12, Lemma 3.26]). Let $\mathcal{C}$ be a pivotal category and $f: X \rightarrow Y$ a morphism. Then:

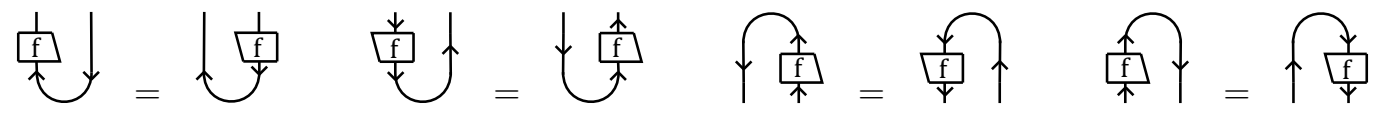

The diagrammatic calculus is summarised by the following theorem.

Theorem 2.7 ([17, Theorem 4.14]). Two diagrams for a morphism in a pivotal category represent the same morphism if there is a planar isotopy between them, which may include sliding of morphisms as in Proposition 2.6.

In a pivotal category we can define notions of dimension for objects and trace for morphisms.

Definition 2.8. Let $X$ be an object and let $f: X \rightarrow X$ be a morphism in a pivotal category $\mathcal{C}$. We define the right trace of $f$ to be the following morphism $\operatorname{Tr}_{R}(f): \mathbb{1} \rightarrow \mathbb{1}$ :

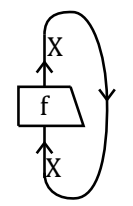

We define the right dimension $\operatorname{dim}_{R}(r)$ of an object $X$ of $\mathcal{C}$ to be $\operatorname{Tr}_{R}\left(\operatorname{id}_{X}\right): \mathbb{1} \rightarrow \mathbb{1}$. The left traces $\operatorname{Tr}_{L}$ and left dimensions $\operatorname{dim}_{L}$ are defined analogously using the right cup and left cap.

Definition 2.9. We call a pivotal category $\mathcal{C}$ spherical if, for object $X$, and any morphism $f: X \rightarrow X$, $\operatorname{Tr}_{L}(f)=\operatorname{Tr}_{R}(f)=: \operatorname{Tr}(f)$. In this case we call $\operatorname{Tr}(f)$ and $\operatorname{dim}(f)$ simply the trace and the dimension. 


\subsubsection{Pivotal dagger categories}

Definition 2.10. A dagger structure on a monoidal category $\mathcal{C}$ is specified by a contravariant identityon-objects $\dagger: \mathcal{C} \rightarrow \mathcal{C}$, written as a power on morphisms, i.e. $\dagger(f)=: f^{\dagger}$, which is:

- Involutive: for any morphism $f: X \rightarrow Y, \dagger(\dagger(f))=f$.

- Compatible with monoidal product: for any objects $X, X^{\prime}, Y, Y^{\prime}$ and morphisms $\alpha: X \rightarrow X^{\prime}$ and $\beta: Y \rightarrow Y^{\prime}$ we have $(\alpha \otimes \beta)^{\dagger}=\alpha^{\dagger} \otimes \beta^{\dagger}$.

We call $f^{\dagger}$ the dagger of $f$.

In the graphical calculus, we represent the dagger of a morphism by reflection in a horizontal axis, preserving the direction of any arrows:

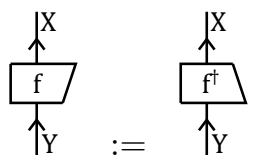

Definition 2.11. Let $\mathcal{C}$ be a dagger category. We say that a morphism $\alpha: X \rightarrow Y$ is an isometry if $\alpha^{\dagger} \circ \alpha=\mathrm{id}_{X}$. We say that it is unitary if it is an isometry and additionally $\alpha \circ \alpha^{\dagger}=\mathrm{id}_{Y}$.

We now give the condition for compatibility of dagger and pivotal structure.

Definition 2.12. Let $\mathcal{C}$ be a pivotal category which is also a monoidal dagger category. We say that $\mathcal{C}$ is pivotal dagger when, for all objects $X$ :

$$
\downarrow=(\uparrow \uparrow)^{\dagger} \quad \uparrow \uparrow=(\downarrow \downarrow)^{\dagger}
$$

Remark 2.13. For any object $X$ in a monoidal dagger category, a right dual $\left[X^{*}, \eta_{X}, \epsilon_{X}\right]$ induces a left dual $\left[X^{*}, \epsilon_{X}^{\dagger}, \eta_{X}^{\dagger}\right]$. This means that a monoidal dagger category with right duals also has left duals. The pivotal structure gives another way to obtain left duals from right duals (9). The equation (12) implies that the left duals obtained from the dagger structure are the same as those obtained from the pivotal structure.

Practically, when taking the dagger of a cup or a cap in a pivotal dagger category, the equation (12) implies we should reflect the cup or cap in a horizontal axis, preserving the direction of the arrows.

For any morphism $f: X \rightarrow Y$, a pivotal dagger structure implies the following conjugate morphism $f^{*}$ is graphically well-defined:

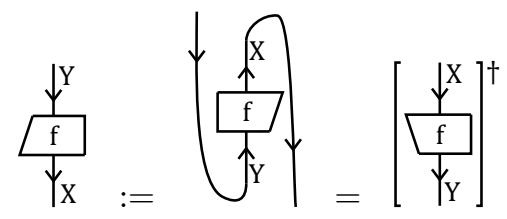

Remark 2.14. Following the bra-ket convention, we draw morphisms $f: \mathbb{1} \rightarrow X$ and $f^{\dagger}: X \rightarrow \mathbb{1}-$ called states and effects of $X$ respectively - as triangles rather than as boxes. The morphisms $f$ and 
$f^{\dagger}$ can be distinguished from $f^{T}$ and $f^{*}$ by the direction of the arrows:

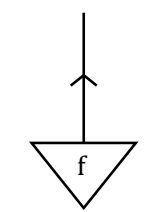

$f: \mathbb{1} \rightarrow X$

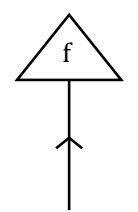

$f^{\dagger}: X \rightarrow \mathbb{1}$

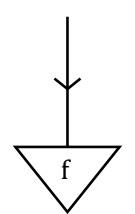

$f^{*}: \mathbb{1} \rightarrow X^{*}$

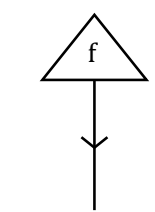

$f^{T}: X^{*} \rightarrow \mathbb{1}$

\subsubsection{Example: the category Hilb}

A basic example of a pivotal dagger category is the category Hilb. The objects of the monoidal category Hilb are finite-dimensional Hilbert spaces, and the morphisms are linear maps between them; composition of morphisms is composition of linear maps. The monoidal product is given on objects by the tensor product of Hilbert spaces, and on morphisms by the tensor product of linear maps; the unit object is the 1-dimensional Hilbert space $\mathbb{C}$.

For any object $H$, its right dual is defined to be the dual Hilbert space $H^{*}$. Any basis $\left\{\left|v_{i}\right\rangle\right\}$ for $H$ defines a cup and cap:
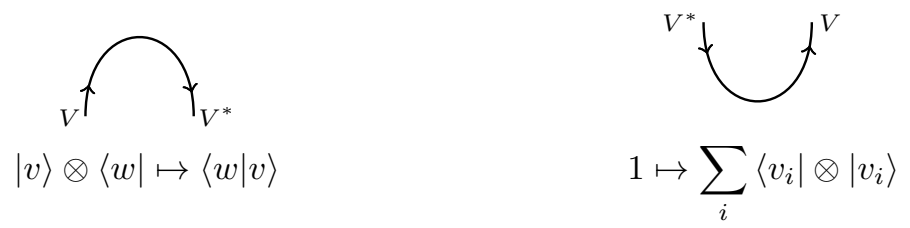

It may easily be checked that this cup and cap fulfil the snake equations (5). This duality is pivotal; the monoidal natural isomorphism from the identity functor to the double duals functor is given by the standard isomorphism from a Hilbert space to its double dual.

The dagger structure is given by the Hermitian adjoint of a linear map. As long as the basis $\left\{\left|v_{i}\right\rangle\right\}$ is orthonormal Hilb is pivotal dagger. The transpose (8) and conjugate (13) are simply the usual transpose and complex conjugate of a linear map with respect to the orthonormal basis defining the duality.

In fact, Hilb is a compact closed category - it is symmetric monoidal in a way which is compatible with its pivotal dagger structure. Because it is symmetric monoidal, diagrams in Hilb should be considered as embedded in four-dimensional space. In particular, for any two Hilbert spaces $V, W$ there is a swap map $\sigma_{V, W}: V \otimes W \rightarrow W \otimes V$. In four dimensions there is no difference between overcrossings and undercrossings, so we simply draw this as an intersection:

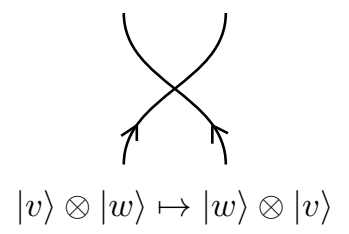

The four-dimensional calculus allows us to untangle arbitrary diagrams and remove any twists, as exemplified by the following equations, which hold regardless of the direction of the arrows on the wires:
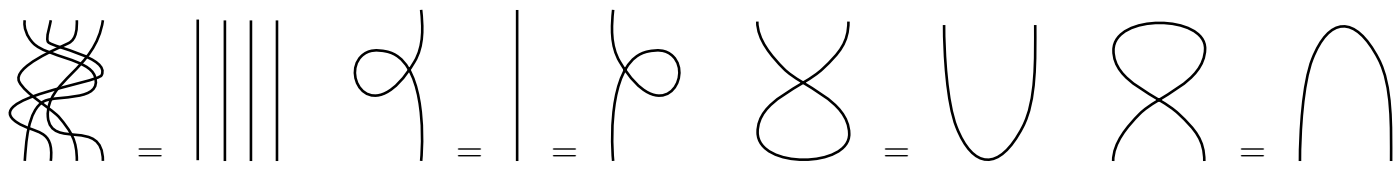

It immediately follows that Hilb is spherical. The trace and dimension of Definition 2.8 reduce to the usual notion of trace and dimension of linear maps and Hilbert spaces. 
The endomorphism algebra. The diagrammatic calculus in Hilb also allows us to conveniently express the endomorphism algebra $B(H)$ of a Hilbert space $H$ using the pivotal dagger structure.

Definition 2.15. Let $H$ be a Hilbert space. We define the following endomorphism *-algebra on $H \otimes H^{*}:$

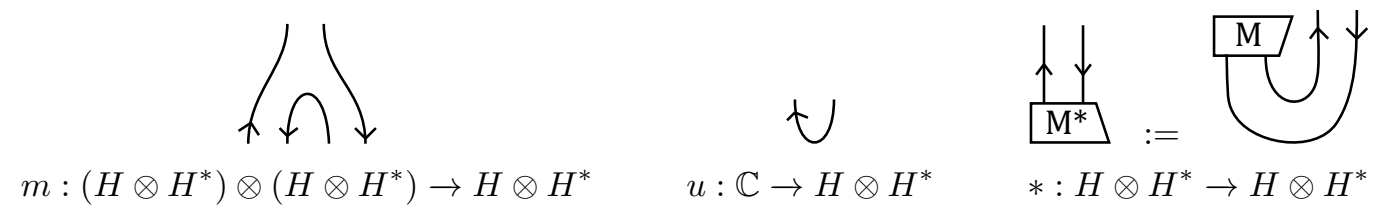

It is straightforward to check that the endomorphism algebra is indeed a $*$-algebra using the diagrammatic calculus of the pivotal dagger category Hilb. In fact, it is a Frobenius monoid (Definition 4.1).

Proposition 2.16. There is a *-isomorphism between the endomorphism algebra $H \otimes H^{*}$ and the *-algebra $B(H)$.

Proof. Consider the linear bijection $H \otimes H^{*} \rightarrow B(H)$ defined on orthonormal basis elements by $|i\rangle \otimes\langle j|\mapsto| i\rangle\langle j|$. It is multiplicative:

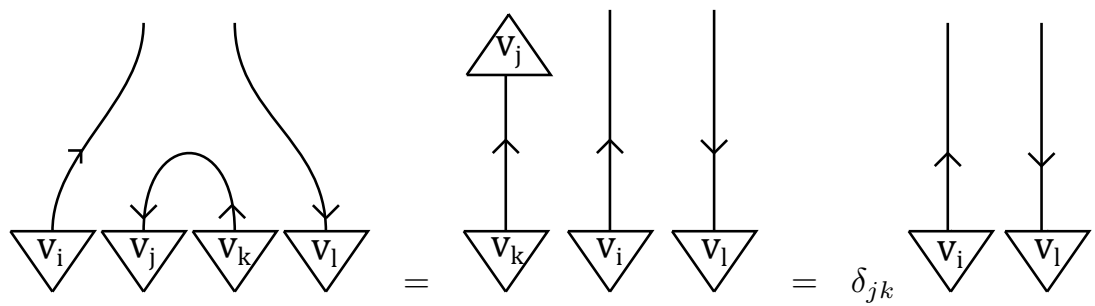

It is also unital, since $\sum_{i}\left|v_{i}\right\rangle\left\langle v_{i}\right|=\mathbb{1}$. Finally, the involution is preserved:

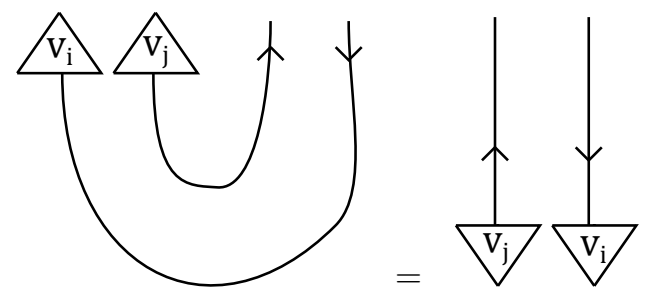

\subsection{Monoidal functors}

\subsubsection{Diagrammatic calculus for monoidal functors}

While our monoidal categories are strict, allowing us to use the diagrammatic calculus, we will consider functors between them which are not strict. For this, we use a graphical calculus of functorial boxes [13].

Definition 2.17. Let $\mathcal{C}, \mathcal{D}$, be monoidal categories. A monoidal functor $F: \mathcal{C} \rightarrow \mathcal{D}$ consists of the following data.

- A functor $F: \mathcal{C} \rightarrow \mathcal{D}$. 
In the graphical calculus, we represent the effect of the functor $F$ by drawing a shaded box around objects and morphisms in $\mathcal{C}$. For example, let $X, Y$ be objects and $f: X \rightarrow Y$ a morphism in $\mathcal{C}$. Then the morphism $F(f): F(X) \rightarrow F(Y)$ in $\mathcal{D}$ is represented as:

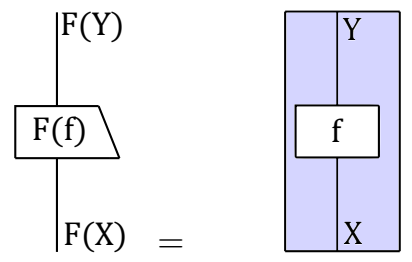

- For every pair of objects $X, Y$ of $\mathcal{C}$, an invertible multiplicator morphism $m_{X, Y}: F(X) \otimes_{D}$ $F(Y) \rightarrow F\left(X \otimes_{C} Y\right)$. In the graphical calculus, these morphisms and their inverses are represented as follows:
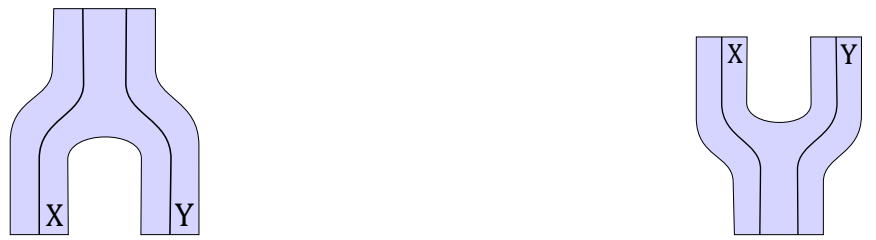

$$
m_{X, Y}: F(X) \otimes_{\mathcal{D}} F(Y) \rightarrow F\left(X \otimes_{\mathcal{C}} Y\right) \quad m_{X, Y}^{-1}: F\left(X \otimes_{\mathcal{C}} Y\right) \rightarrow F(X) \otimes_{\mathcal{D}} F(Y)
$$

- An invertible 'unitor' morphism $u: \mathbb{1}_{D} \rightarrow F\left(\mathbb{1}_{C}\right)$. In the diagrammatic calculus, this morphism and its inverse are represented as follows (recall that the monoidal unit is invisible in the diagrammatic calculus):

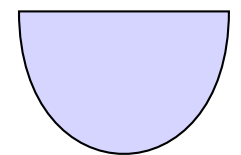

$u: \mathbb{1}_{D} \rightarrow F\left(\mathbb{1}_{C}\right)$

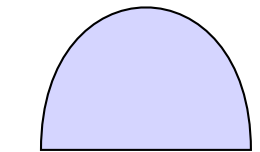

$$
u^{-1}: F\left(\mathbb{1}_{C}\right) \rightarrow \mathbb{1}_{D}
$$

The multiplicators and unitor obey the following coherence equations:

- Naturality. For any objects $X, X^{\prime}, Y, Y^{\prime}$ and morphisms $f: X \rightarrow X^{\prime}, g: Y \rightarrow Y^{\prime}$ in $\mathcal{C}$ :

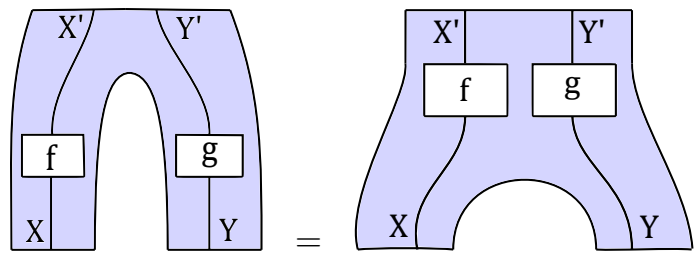

- Associativity. For any objects $X, Y, Z$ of $\mathcal{C}$ :

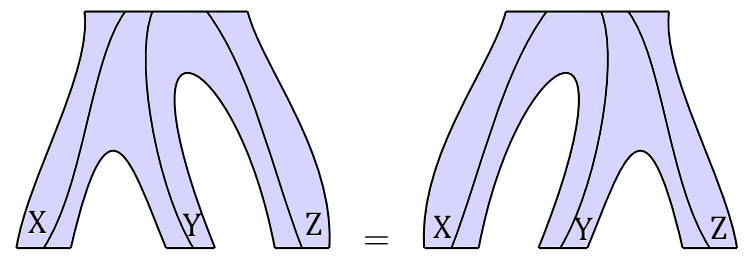


- Unitality. For any object $X$ of $\mathcal{C}$ :

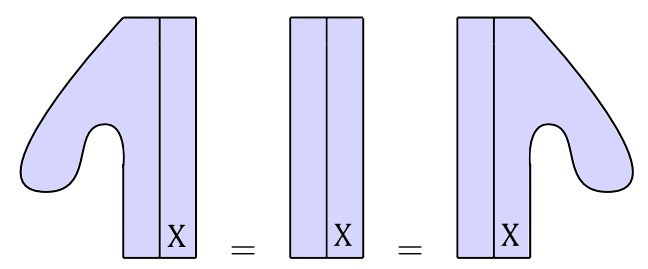

For monoidal dagger categories $\mathcal{C}, \mathcal{D}$, we say that a monoidal functor $F: \mathcal{C} \rightarrow \mathcal{D}$ is unitary if $F\left(f^{\dagger}\right)=$ $F(f)^{\dagger}$ and the multiplicators and unitor are unitary morphisms.

We observe that the analogous conaturality, coassociativity and counitality equations for the inverses $\left\{m_{X, Y}^{-1}\right\}, u^{-1}$, obtained by reflecting (26-28) in a horizontal axis, are already implied by (26-28). To give some idea of the calculus of functorial boxes, we explicitly prove the following lemma and proposition. From now on we will unclutter the diagrams by omitting object labels, unless adding the labels seems to significantly aid comprehension.

Lemma 2.18. For any objects $X, Y, Z$ of $\mathcal{C}$, the following equations are satisfied:
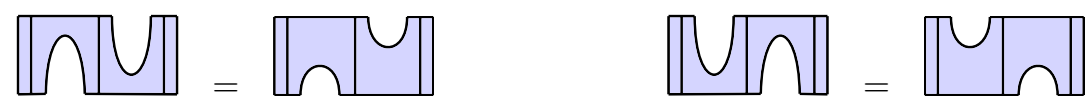

Proof. We prove the left equation; the right equation is proved similarly.

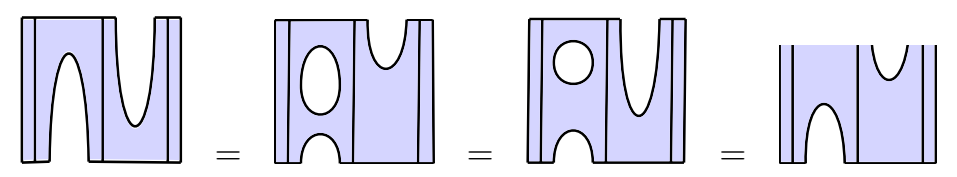

Here the first and third equalities are by invertibility of $m_{X, Y}$, and the second is by coassociativity.

With Lemma 2.18, the equations (26-28) are sufficient to deform functorial boxes topologically as required. From now on we will do this mostly without comment.

\subsubsection{Induced duals}

We first observe that the duals in $\mathcal{C}$ induce duals in $\mathcal{D}$ under a monoidal functor $F: \mathcal{C} \rightarrow \mathcal{D}$.

Proposition 2.19 (Induced duals). Let $X$ be an object in $\mathcal{C}$ and $\left[X^{*}, \eta, \epsilon\right]$ a right dual. Then $F\left(X^{*}\right)$ is a right dual of $F(X)$ in $\mathcal{D}$ with the following cup and cap:

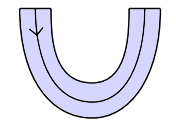

$F(\eta)$

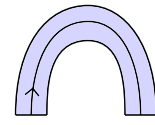

$F(\epsilon)$

The analogous statement holds for left duals.

Proof. We show one of the snake equations (5) in the case of right duals; the others are all proved similarly.

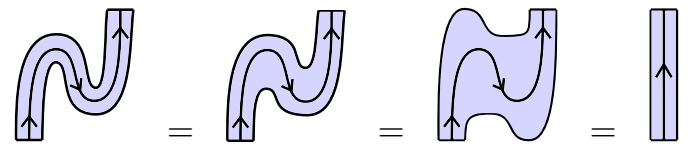

Here the first equality is by Lemma 2.18 , the second by (26) and the third by (28) and the right snake equation in $\mathcal{C}$. 
For any 1-morphism $X$ of $\mathcal{C}$, then, we have two sets of left and right duals on $F(X)$; the first from the pivotal structure in $\mathcal{C}$ by Proposition 2.19, and the second from the pivotal structure in $\mathcal{D}$.

In the diagrammatic calculus we distinguish between these two duals by drawing a large downwards arrowhead on the dual in $\mathcal{D}$, like so:

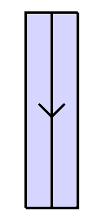

Induced duals $F\left(X^{*}\right)$ from $\mathcal{C}$

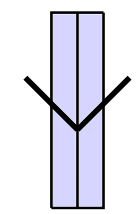

Duals $F(X)^{*}$ in $\mathcal{D}$

\subsection{Compact quantum groups}

In this work we will restrict our attention to the specific case of UPTs between unitary $\mathbb{C}$-linear monoidal functors from $C^{*}$-tensor categories with conjugates $\mathcal{C}$ into the category Hilb of finite-dimensional Hilbert spaces and linear maps. Provided that such a functor exists, there is a duality theory which identifies $\mathcal{C}$ as the category of corepresentations of a certain algebraic object.

\subsection{1 $C^{*}$-tensor categories}

We first recall the definition of a $C^{*}$-tensor category with conjugates.

Definition 2.20. A dagger category is $\mathbb{C}$-linear if:

- For every pair of objects $X, Y, \operatorname{Hom}(X, Y)$ is a complex vector space.

- For every triple of objects $X, Y, Z$, composition $\circ: \operatorname{Hom}(X, Y) \times \operatorname{Hom}(Y, Z) \rightarrow \operatorname{Hom}(X, Z)$ is a bilinear map.

- For every pair of objects $X, Y$, the dagger $\dagger: \operatorname{Hom}(X, Y) \rightarrow \operatorname{Hom}(Y, X)$ is an antilinear and positive map, i.e. $\alpha \circ \alpha^{\dagger}=0$ iff $\alpha=0$.

A monoidal dagger category is $\mathbb{C}$-linear if, additionally:

- For every quadruple of objects $X, X^{\prime}, Y, Y^{\prime}$, the tensor product $\otimes: \operatorname{Hom}\left(X, X^{\prime}\right) \times \operatorname{Hom}\left(Y, Y^{\prime}\right) \rightarrow$ $\operatorname{Hom}\left(X \otimes X^{\prime}, Y \otimes Y^{\prime}\right)$ is a bilinear map.

A functor $F: \mathcal{C} \rightarrow \mathcal{D}$ between $\mathbb{C}$-linear categories is called $\mathbb{C}$-linear if the induced maps on Hom-spaces $F_{X, Y}: \operatorname{Hom}_{\mathcal{C}}(X, Y) \rightarrow \operatorname{Hom}_{\mathcal{D}}(F(X), F(Y))$ are $\mathbb{C}$-linear.

Definition 2.21. A $\mathbb{C}$-linear monoidal dagger category is called a $C^{*}$-tensor category if

- $\operatorname{Hom}(X, Y)$ is a Banach space for all objects $X, Y$, and $\|f g\| \leq\|f\|\|g\|$.

- The dagger satisfies the following properties for any morphism $f: X \rightarrow Y$ :

- $\left\|f^{\dagger} \circ f\right\|=\|f\|^{2}$; in particular, $\operatorname{End}(X)$ is a $C^{*}$-algebra for any object $X$.

$-f^{\dagger} \circ f$ is positive as an element of the $C^{*}$-algebra $\operatorname{End}(X)$.

Following [16] we also assume that our $C^{*}$-tensor categories have the following completeness properties:

- There is an object $\mathbf{0}$ such that $\operatorname{dim}(\operatorname{Hom}(\mathbf{0}, X))=0$ for every object $X$.

- There are binary direct sums: for every pair of objects $X_{1}, X_{2}$, there is an object $X_{1} \oplus X_{2}$ and morphisms $s_{i}: X_{i} \rightarrow X_{1} \oplus X_{2}$ (for $i \in\{1,2\}$ ) such that

$$
s_{i}^{\dagger} s_{i}=\operatorname{id}_{X_{i}} s_{1}^{\dagger}+s_{2} s_{2}^{\dagger}=\operatorname{id}_{X_{1} \oplus X_{2}}
$$


- Dagger idempotents split: for any morphism $f: X \rightarrow X$ such that $f^{\dagger}=f \circ f=f$, there exists an object $Y$ and an isometry $\iota: Y \rightarrow X$ such that $\iota \circ \iota^{\dagger}=f$.

- The unit object $\mathbb{1}$ is irreducible, i.e. $\operatorname{End}(\mathbb{1})=\mathbb{C i d}_{\mathbb{1}}$.

In the setting of $C^{*}$-tensor categories, one normally speaks of conjugates ${ }^{1}$ rather than duals.

Definition 2.22. Let $X$ be an object of a $C^{*}$-tensor category $\mathcal{C}$. We say that morphisms $R: \mathbb{1} \rightarrow$ $X^{*} \otimes X$ and $\bar{R}: \mathbb{1} \rightarrow X \otimes X^{*}$ are solutions to the conjugate equations if $\left[X^{*}, R, \bar{R}^{\dagger}\right]$ is right dual to $X$.

We observe that since $\left[X^{*}, R, \bar{R}^{\dagger}\right]$ is right dual to $X$, then $\left[X^{*}, \bar{R}, R^{\dagger}\right]$ is left dual to $X$. We therefore have left and right duals for $X$ satisfying the equations (12). Suppose that solutions to the conjugate equations are chosen for every object in $\mathcal{C}$. For $\mathcal{C}$ to be a pivotal dagger category with right and left duals $\left[X^{*}, R_{X}, \bar{R}_{X}^{\dagger}\right]$ and $\left[X^{*}, \bar{R}_{X}, R_{X}^{\dagger}\right]$, by (9) we require a monoidal natural isomorphism $\iota$ from the double duals functor to the identity functor relating the left and right duals, i.e.:

$$
\bar{R}_{X}=\left(\iota_{X} \otimes \mathrm{id}_{X}^{*}\right) R_{X^{*}} \quad R_{X}^{\dagger}=\bar{R}_{X^{*}}^{\dagger}\left(\mathrm{id}_{X^{*}} \otimes \iota_{X}^{-1}\right)
$$

In any $C^{*}$-tensor category with conjugates each object possesses a distinguished conjugate $\left[X^{*}, R, \bar{R}\right]$, unique up to unitary isomorphism, called a standard solution [16, Def 2.2.14].

Theorem 2.23. Let $\mathcal{C}$ be a $C^{*}$-tensor category with conjugates, and let $\left\{\left[X^{*}, R_{X}, \bar{R}_{X}\right]\right\}$ be a choice of standard solutions for all objects $X$ in $\mathcal{C}$. Then there exists a unitary monoidal natural isomorphism $\iota$ from the double duals functor to the identity functor, such that the equations (31) are obeyed.

Proof. It is shown in [16, Thm. 2.2.21] that such a unitary natural isomorphism $\iota$ exists. What remains is to demonstrate that $\iota$ is monoidal. We provide a proof of this fact in the Appendix (Section 6).

A $C^{*}$-tensor category equipped with standard solutions to the conjugate equations is therefore a pivotal dagger category (in fact, a spherical dagger category [16, Thm 2.2.16]) and can be treated using the graphical calculus just discussed.

Definition 2.24. Let $\mathcal{C}$ be a $C^{*}$-tensor category with conjugates. We call a unitary $\mathbb{C}$-linear monoidal functor $F: \mathcal{C} \rightarrow$ Hilb a fibre functor.

\subsubsection{Compact quantum groups}

We now introduce the algebraic objects dual to $C^{*}$-tensor categories with conjugates and a chosen fibre functor. All algebras are taken over $\mathbb{C}$.

Definition 2.25 ([16, Definition 1.6.1]). A unital $*$-algebra $A$ equipped with a unital $*$-homomorphism $\Delta: A \rightarrow A \otimes A$ (the comultiplication) is called a Hopf-*-algebra if $\left(\Delta \otimes \operatorname{id}_{A}\right) \circ \Delta=\left(\operatorname{id}_{A} \otimes \Delta\right) \circ \Delta$ and there exist linear maps $\epsilon: A \rightarrow \mathbb{C}$ (the counit) and $S: A \rightarrow A$ (the antipode) such that
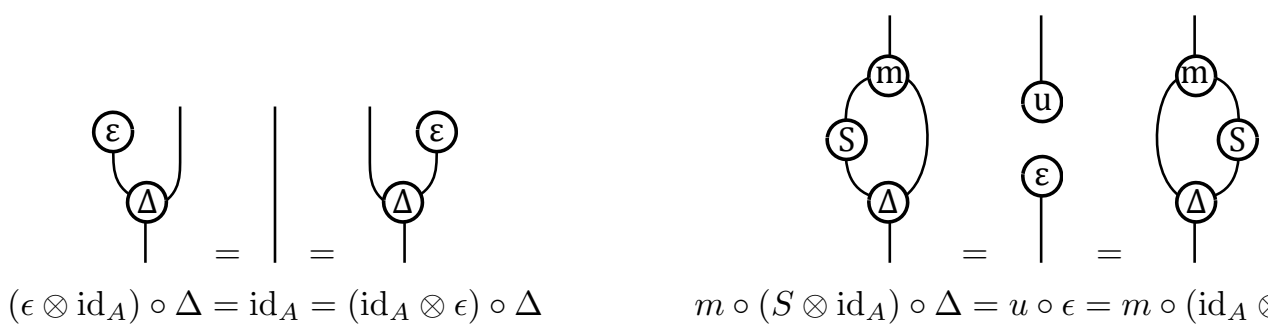

$$
\left(\epsilon \otimes \operatorname{id}_{A}\right) \circ \Delta=\operatorname{id}_{A}=\left(\operatorname{id}_{A} \otimes \epsilon\right) \circ \Delta
$$

$$
m \circ\left(S \otimes \operatorname{id}_{A}\right) \circ \Delta=u \circ \epsilon=m \circ\left(\operatorname{id}_{A} \otimes S\right) \circ \Delta(32)
$$

where $m: A \otimes A \rightarrow A$ is the multiplication and $u: \mathbb{C} \rightarrow A$ the unit of the algebra $A$.

\footnotetext{
${ }^{1}$ Note that these are not the conjugates of (13); they are rather dual objects.
} 
Definition 2.26 ([16, Definition 1.6.5]). A corepresentation of a Hopf $*$-algebra $A$ on a vector space $H$ is a linear map $\delta: H \rightarrow H \otimes A$ such that

$$
\left(\delta \otimes \operatorname{id}_{A}\right) \circ \delta=\left(\operatorname{id}_{H} \otimes \Delta\right) \circ \delta \quad\left(\operatorname{id}_{H} \otimes \epsilon\right) \circ \delta=\operatorname{id}_{H}
$$

The corepresentation is called unitary if $H$ is a Hilbert space and

$$
\langle\delta(\xi), \delta(\zeta)\rangle=(\xi, \zeta) 1_{A} \quad \text { for all } \xi, \zeta \in H
$$

where the $A$-valued inner product $\langle\cdot, \cdot\rangle$ on $H \otimes A$ is defined by linear extension of $\langle\xi \otimes a, \zeta \otimes b\rangle=$ $(\xi, \zeta) a^{*} b$.

For $\left(H_{1}, \delta_{1}\right),\left(H_{2}, \delta_{2}\right)$ corepresentations of a Hopf-*-algebra $A$, we say that a linear map $f: H_{1} \rightarrow H_{2}$ is an intertwiner $f:\left(H_{1}, \delta_{1}\right) \rightarrow\left(H_{2}, \delta_{2}\right)$ if $\delta_{2} \circ f=\left(f \otimes \mathrm{id}_{A}\right) \circ \delta_{1}$.

Definition 2.27. Let $(H, \delta)$ be a finite-dimensional unitary corepresentation of $A$, and let $\left\{\left|v_{i}\right\rangle\right\}$ be an orthonormal basis of $H$. Then $\left(\left\langle v_{j}\right| \otimes \operatorname{id}_{A}\right) \circ \delta\left(\left|v_{i}\right\rangle\right)$ defines an $A$-valued matrix $U_{i j}$; we say that the entries of this matrix are the matrix coefficients of the representation in the basis $\left\{\left|v_{i}\right\rangle\right\}$.

Definition 2.28 (c.f. [16, Theorem 1.6.7]). We say that a Hopf-*-algebra is a compact quantum group algebra (CQG algebra) if it is generated as an algebra by matrix coefficients of its finite-dimensional unitary corepresentations.

For a compact quantum group algebra, a monoidal product of corepresentations can be defined [16, Definition 1.3.2], as can a notion of conjugate corepresentation [16, Def. 1.4.5]. This yields a $C^{*}$-tensor category with conjugates $\operatorname{Corep}(A)$ whose objects are finite-dimensional unitary corepresentations of the algebra $(A, \Delta)$ and whose morphisms are intertwiners, with an obvious canonical fibre functor $F: \operatorname{Corep}(A) \rightarrow$ Hilb which forgets the representation. Taking standard solutions to the conjugate equations, $\operatorname{Corep}(A)$ has the structure of a pivotal dagger category.

Such an algebra is considered as the algebra of matrix coefficents of representations of some 'compact quantum group' $G$, such that $\operatorname{Rep}(G)=\operatorname{Corep}(A)$. We will refer to compact quantum groups $G$, and write $\operatorname{Rep}(G)$, in order to emphasise the similarity with representation theory of compact groups. However, the algebra $A_{G}$ is the concrete object in general.

We now recall the theorem relating $C^{*}$-tensor categories with conjugates to compact quantum groups.

Theorem 2.29 ([16, Theorem 2.3.2]). Let $\mathcal{C}$ be a $C^{*}$-tensor category with conjugates, and let $U: \mathcal{C} \rightarrow$ Hilb be a fibre functor. Then there exists a compact quantum group algebra $A$ (uniquely determined up to isomorphism) and a unitary monoidal equivalence $E_{U}: \mathcal{C} \rightarrow \operatorname{Rep}\left(G_{A}\right)$, such that $U$ is unitarily monoidally naturally isomorphic to $F \circ E_{U}$.

\subsection{Unitary pseudonatural transformations}

A pseudonatural transformation between pseudofunctors is a 2-categorical generalisation of a natural isomorphism between functors [10]. Monoidal functors are pseudofunctors, where we consider a monoidal category as a 2-category with a single object. Pseudonatural transformations between monoidal functors can be seen as a generalisation of monoidal natural isomorphisms.

\subsubsection{Definition}

If the categories $\mathcal{C}$ and $\mathcal{D}$ are pivotal dagger categories, there is a notion of unitarity for pseudonatural transformations between monoidal functors $\mathcal{C} \rightarrow \mathcal{D}$ which generalises unitary monoidal natural isomorphism [19]. We here deal only with unitary pseudonatural transformations. 
Definition 2.30. Let $\mathcal{C}, \mathcal{D}$ be pivotal dagger categories, and let $F_{1}, F_{2}: \mathcal{C} \rightarrow \mathcal{D}$ be unitary monoidal functors. (We colour the functorial boxes blue and red respectively.) A unitary pseudonatural transformation $(\alpha, H): F_{1} \rightarrow F_{2}$ is defined by the following data:

- An object $H$ of $\mathcal{D}$ (drawn as a green wire).

- For every object $X$ of $\mathcal{C}$, a unitary morphism $\alpha_{X}: F_{1}(X) \otimes H \rightarrow H \otimes F_{2}(X)$ (drawn as a white vertex):

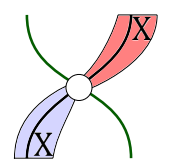

The unitary morphisms $\alpha_{X}$ must satisfy the following conditions:

- Naturality. For every morphism $f: X \rightarrow Y$ in $\mathcal{C}$ :

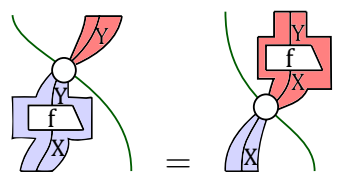

- Monoidality.

- For every pair of objects $X, Y$ of $\mathcal{C}$ :

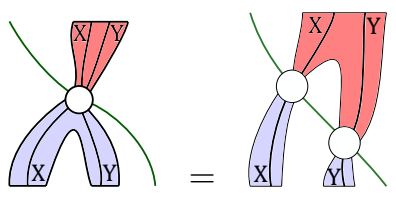

$-\alpha_{\mathbb{1}}$ is defined as follows:

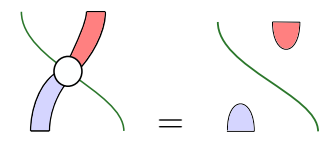

Remark 2.31. Unitary pseudonatural transformations generalise the notion of unitary monoidal natural isomorphism, which we recover when $H \cong \mathbb{1}$.

Remark 2.32. The diagrammatic calculus shows that pseudonatural transformation is a planar notion. The $H$-wire forms a boundary between two regions of the $\mathcal{D}$-plane, one in the image of $F$ and the other in the image of $G$. By pulling through the $H$-wire, morphisms from $\mathcal{C}$ can move between the two regions (35).

UPTs $(\alpha, H): F_{1} \rightarrow F_{2}$ and $\left(\beta, H^{\prime}\right): F_{2} \rightarrow F_{3}$ can be composed associatively to obtain a UPT $\left(\alpha \otimes \beta, H \otimes H^{\prime}\right): F_{1} \rightarrow F_{3}$ whose components $(\alpha \otimes \beta)_{X}$ are as follows (we colour the $H^{\prime}$-wire orange, and the $F_{3}$-box brown):

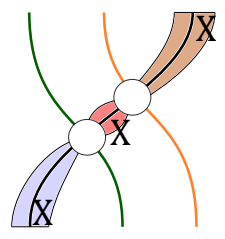

There are also morphisms between pseudonatural transformations, known as modifications [10]. 
Definition 2.33. Let $(\alpha, H),\left(\beta, H^{\prime}\right): F_{1} \Rightarrow F_{2}$ be UPTs. (We colour the $H$-wire green and the $H^{\prime}$ wire orange.) A modification $f: \alpha \rightarrow \beta$ is a morphism $f: H \rightarrow H^{\prime}$ satisfying the following equation for all components $\left\{\alpha_{X}, \beta_{X}\right\}$ :

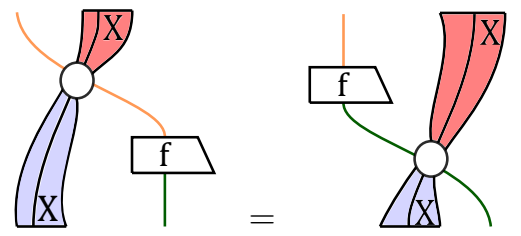

Modifications can themselves be composed horizontally and vertically — vertical composition is composition of morphisms in $\mathcal{D}$, while horizontal composition is monoidal product of morphisms in $\mathcal{D}$. The dagger of a modification is also a modification. Altogether, the compositional structure is that of a dagger 2-category, i.e. a 2-category with a dagger on 2-cells which is compatible with horizontal composition. (For an explicit definition, see [7, Def. 2.5] or [19, Def. 2.13].)

Definition 2.34. Let $\mathcal{C}, \mathcal{D}$ be pivotal dagger categories. The dagger 2-category $\operatorname{Fun}(\mathcal{C}, \mathcal{D})$ is defined as follows:

- Objects: unitary monoidal functors $F_{1}, F_{2}, \ldots, \cdot: \mathcal{C} \rightarrow \mathcal{D}$.

- 1-morphisms: unitary pseudonatural transformations $\alpha, \beta, \cdots: F_{1} \rightarrow F_{2}$.

- 2-morphisms: modifications $f, g, \cdots: \alpha \rightarrow \beta$.

Because we are able to assume that $\mathcal{C}$ and $\mathcal{D}$ are strict, $\operatorname{Fun}(\mathcal{C}, \mathcal{D})$ is a strict 2-category.

Remark 2.35. Because $\operatorname{Fun}(\mathcal{C}, \mathcal{D})$ is a dagger 2-category, the endomorphism categories $\operatorname{End}(F)$ of UPTs $F \rightarrow F$ and modifications for a given functor $F$ are monoidal dagger categories.

\subsubsection{Duals}

Definition 2.36. Let $(\alpha, H): F_{1} \rightarrow F_{2}$ be a UPT. Then the $d u a l$ of $\alpha$ is a UPT $\left(\alpha^{*}, H^{*}\right): F_{2} \rightarrow F_{1}$ whose components $\alpha_{X}^{*}$ are defined as follows:

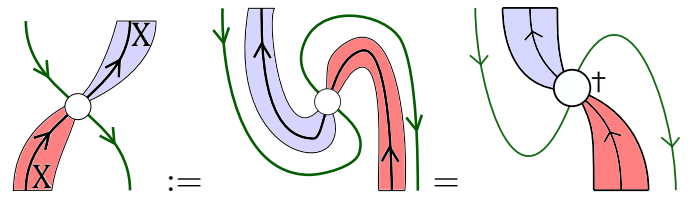

Here the second equality is by unitarity of $\alpha$ [19, Prop. 5.2]. We sometimes put a $*$ next to the vertex for the dual UPT $\alpha^{*}$ to distinguish it from $\alpha$, although the orientation of the $H$-wire is sufficient for this.

For the composition of a UPT with its dual, the cups and caps of the dagger duality in $\mathcal{D}$ are modifications [19, Thm. 4.4, Cor. 5.6] :
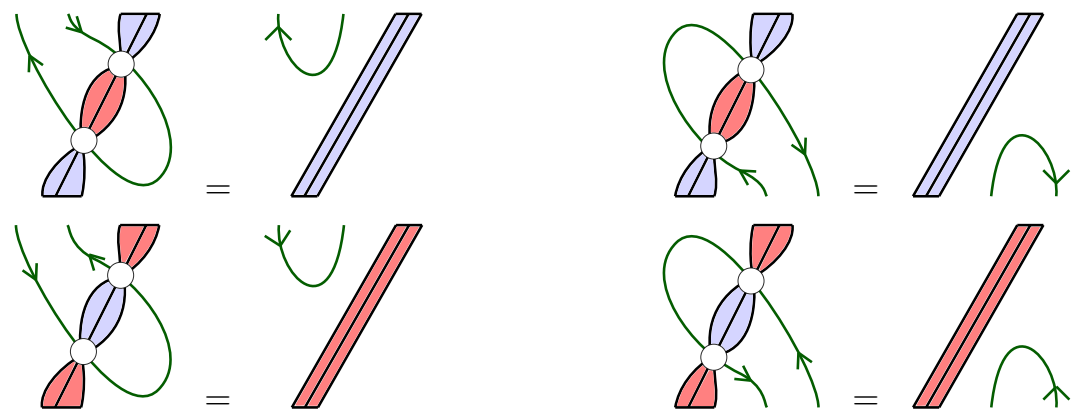


\section{UPTs and Hopf-Galois theory}

The Tannaka duality relating $C^{*}$-tensor categories to compact quantum group algebras extends to the characterisation of their fibre functors. For a compact quantum group $G$, the algebraic objects corresponding to fibre functors on $\operatorname{Rep}(G)$ are Hopf-Galois objects for the CQG algebra $A_{G}$, also known as noncommutative torsors. In this section we will show that UPTs between fibre functors can be classified in terms of the finite-dimensional representation theory of these Hopf-Galois objects.

\subsection{Background on Hopf-Galois theory}

We now review the basics of Hopf-Galois theory for compact quantum groups, based on [3].

Definition 3.1. Let $A$ be a CQG algebra, and $Z$ a $*$-algebra. We say that a left corepresentation $\delta: Z \rightarrow A \otimes Z$ is a left coaction if $\delta$ is a $*$-homomorphism.

Definition 3.2. [3, Def. 4.1.1] Let $A$ be a CQG algebra, and let $Z$ be an $*$-algebra with a left $A$-coaction. We say that $Z$ is a left $A$-Hopf-Galois object if the following linear map is a bijection:

$$
\left(\operatorname{id}_{A} \otimes m_{Z}\right) \circ\left(\delta \otimes \operatorname{id}_{Z}\right): Z \otimes Z \rightarrow A \otimes Z
$$

Right $A$-Hopf-Galois objects may be defined similarly. For two CQG algebras $A_{1}, A_{2}$, if $Z$ is a left $A_{1^{-}}$ and a right $A_{2}$-Hopf Galois object, we say that it is an $A_{1}-A_{2}$-bi-Hopf-Galois object.

From any left Hopf-Galois object for $A_{G}$ it is possible to construct a unitary fibre functor on $\operatorname{Rep}(G)$.

Construction 3.3 ([3, Prop. 4.3.1]). Let $G$ be a compact quantum group, and $\left(Z, \delta_{Z}\right)$ a left HopfGalois object for $A_{G}$. Then a unitary fibre functor $F_{Z}: \operatorname{Rep}(G) \rightarrow$ Hilb may be constructed, part of whose definition is as follows:

- On objects. For any corepresentation $\left(V, \delta_{V}\right)$ of $A_{G}$, as a vector space $F(V)=V \wedge Z$, where $V \wedge Z$ is the equaliser of the double arrow:

$$
\delta_{V} \otimes \operatorname{id}_{Z}, \operatorname{id}_{V} \otimes \delta_{Z}: V \otimes Z \rightrightarrows V \otimes A_{G} \otimes Z
$$

- On morphisms. For any intertwiner $f: V \rightarrow W, F(f)=f \wedge \mathrm{id}_{Z}: V \wedge Z \rightarrow W \wedge Z$, where $f \wedge \operatorname{id}_{Z}$ is the map on equalisers induced by $f \otimes \mathrm{id}_{Z}$.

Remark 3.4. The Hopf-Galois object corresponding to the canonical fibre functor is $A_{G}$ itself.

Likewise, from any unitary fibre functor on $\operatorname{Rep}(G)$ one can construct an left $A_{G}$-Hopf-Galois object.

Construction 3.5 (See [3, Prop. 4.3.3] for part of the proof). Let $\mathcal{C}$ be a rigid $C^{*}$-tensor category with conjugates, let $F_{1}, F_{2}: \mathcal{C} \rightarrow$ Hilb be two unitary fibre functors, and let $G_{1}, G_{2}$ be the two compact quantum groups obtained by Tannaka reconstruction (Theorem 2.29). The predual $\operatorname{Hom}^{\vee}\left(F_{1}, F_{2}\right)$ of the vector space $\operatorname{Hom}\left(F_{1}, F_{2}\right)$ of natural transformations $F_{1} \rightarrow F_{2}$ has the structure of an $A_{G_{2}}-A_{G_{1}}{ }^{-}$ bi-Hopf-Galois object.

These constructions lead to a classification of unitary fibre functors on $\operatorname{Rep}(G)$. Let $\operatorname{Gal}(A)$ be the category whose objects are left $A$-Hopf-Galois objects and whose morphisms are $*$-homomorphisms intertwining the $A$-coactions. Let $\operatorname{Fib}(G)$ be the category whose objects are unitary fibre functors on $\operatorname{Rep}(G)$ and whose morphisms are unitary monoidal natural isomorphisms.

Theorem 3.6 ([3, Thm 4.3.4]). Constructions 3.3 and 3.5 yield an equivalence of categories $\operatorname{Gal}(A) \simeq$ $\operatorname{Fib}(G)$.

We also note the following fact characterising the spectrum of a bi-Hopf-Galois object, which Theorem 3.14 will generalise to all finite-dimensional $*$-representations.

Proposition 3.7 (For part of the proof see [3, Thm 4.4.1]). Let $\mathcal{C}$ be a $C^{*}$-tensor category with conjugates, let $F_{1}, F_{2}: \mathcal{C} \rightarrow$ Hilb be fibre functors, and let $Z$ be the corresponding $A_{G_{2}}-A_{G_{1}}$-bi-HopfGalois object. Then there is a bijection between the set of unitary monoidal natural isomorphisms $F_{1} \rightarrow F_{2}$ and the set of 1-dimensional $*$-representations of $Z$. 
A generators-and-relations description of $Z$. We will need the following generators-and-relations description of the bi-Hopf-Galois object $Z=\operatorname{Hom}^{\vee}\left(F_{1}, F_{2}\right)$ linking two fibre functors $F_{1}, F_{2}: \mathcal{C} \rightarrow$ Hilb, taken from $[3,9]$. We assume that $\mathcal{C}$ is a small category.

Consider the vector space $\bigoplus_{V \in \operatorname{Obj}(\mathcal{C})} \operatorname{Hom}\left(F_{2}(V), F_{1}(V)\right)$, where the sum is taken over all objects of $\mathcal{C}$. Let $\mathcal{N}$ be the following subspace:

$$
\left\langle F_{1}(f) \circ v-v \circ F_{2}(f) \quad \mid \quad \forall W \in \operatorname{Obj}(\mathcal{C}), \forall f \in \operatorname{Hom}_{\mathcal{C}}(V, W), \forall v \in \operatorname{Hom}\left(F_{2}(W), F_{1}(V)\right)\right\rangle
$$

Then, as a vector space:

$$
\operatorname{Hom}^{\vee}\left(F_{1}, F_{2}\right):=\bigoplus_{V \in \operatorname{Obj}(\mathcal{C})} \operatorname{Hom}\left(F_{2}(V), F_{1}(V)\right) / \mathcal{N}
$$

We denote $v \in \operatorname{Hom}\left(F_{2}(V), F_{1}(V)\right)$ by $[V, v]$ as an element of $\operatorname{Hom}^{\vee}\left(F_{1}, F_{2}\right)$, which is clearly generated as a vector space by all the $[V, v]$ up to the relations (43). The algebra structure is defined as follows on the generators, where we draw $F_{1}$ with a blue box and $F_{2}$ with a red box:

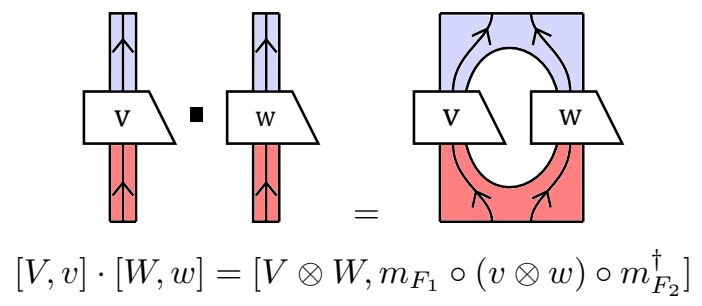

The unit of the algebra is $\left[\mathbb{1}, u_{1} \circ u_{2}^{\dagger}\right]$, where $u_{i}$ is the unitor for $F_{i}$.

The involution is defined as follows on the generators:

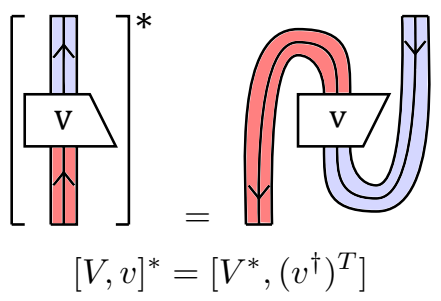

\subsection{UPTs as $*$-representations of Hopf-Galois objects}

We have just recalled that, for a compact quantum group $G$, the category $\operatorname{Fib}(G)$ of fibre functors on the pivotal dagger category $\operatorname{Rep}(G)$ and unitary monoidal natural isomorphisms is characterised by Hopf-Galois theory. In [19] we showed that $\operatorname{Fib}(G)$ can be generalised to a dagger 2-category $\operatorname{Fun}(\operatorname{Rep}(G)$, Hilb) of fibre functors, unitary pseudonatural transformations and modifications. We will now see that this dagger 2-category is also characterised by Hopf-Galois theory: UPTs correspond to finite-dimensional $*$-representations of bi-Hopf-Galois objects, just as unitary monoidal natural transformations correspond to their one-dimensional $*$-representations.

Let $\mathcal{C}$ be a $C^{*}$-tensor category with conjugates, let $F_{1}, F_{2}: \mathcal{C} \rightarrow$ Hilb be fibre functors, and let $Z$ be the corresponding $A_{G_{2}}-A_{G_{1}}$-bi-Hopf-Galois object. We will first show that for every unitary pseudonatural transformation $(\alpha, H): F_{1} \rightarrow F_{2}$, one can construct a $*$-representation $\pi_{\alpha}: Z \rightarrow B(H)$.

Construction 3.8. Recall the generators-and-relations description of $Z$ from Section 3.1. We define the following map $\pi_{\alpha}: \oplus_{V \in \operatorname{Obj}(\mathcal{C})} \operatorname{Hom}\left(F_{2}(V), F_{1}(V)\right) \rightarrow H \otimes H^{*} \cong B(H)$ by its action on generators 
$[V, v]:$

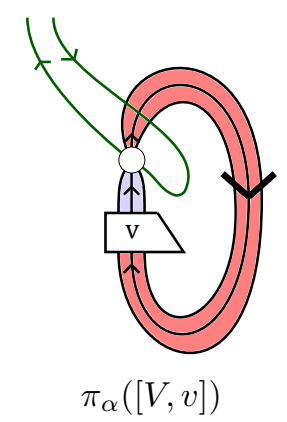

Note that here we are taking the trace with respect to the dual $F_{2}(V)^{*}$ in Hilb (see (29)).

Proposition 3.9. Construction 3.8 defines a $*$-representation $\pi_{\alpha}: Z \rightarrow B(H)$. Moreover, any modification $f:(\alpha, H) \rightarrow\left(\beta, H^{\prime}\right)$ induces an intertwiner $H \rightarrow H^{\prime}$.

Proof. We first show that $\pi_{\alpha}$ induces a well-defined map on $Z=\oplus_{V \in \operatorname{Obj}(\mathcal{C})} \operatorname{Hom}\left(F_{2}(V), F_{1}(V)\right) / \mathcal{N}$, where $\mathcal{N}$ is the subspace defined in (43). For this it is sufficient to show that, for any $f: V \rightarrow W$ in $\mathcal{C}$ and $x: F_{2}(W) \rightarrow F_{1}(V)$ in $\mathcal{D}$, we have $\pi_{\alpha}\left(\left[V, x \circ F_{2}(f)\right]\right)=\pi_{\alpha}\left(\left[W, F_{1}(f) \circ x\right]\right)$ :
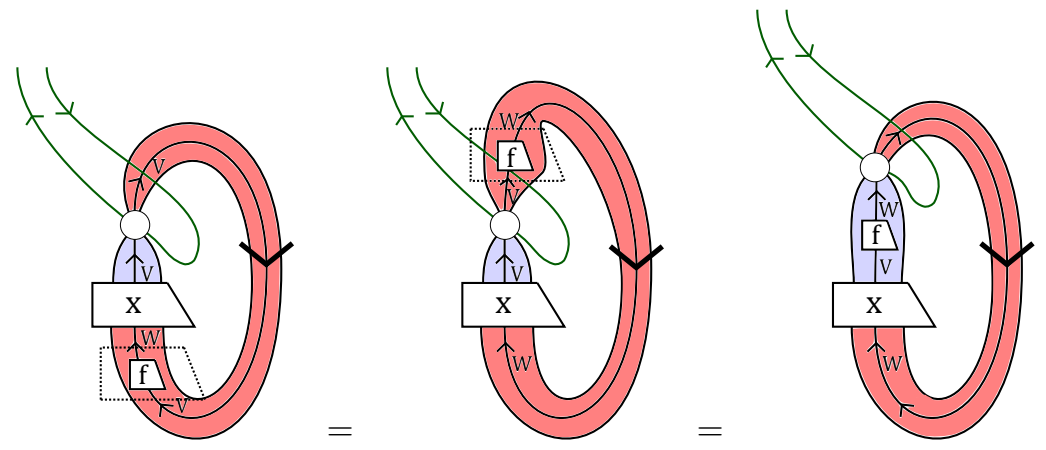

Here for the first equality we slide the morphism $F_{2}(f): F_{2}(V) \rightarrow F_{2}(W)$ in the dotted box around the loop using the graphical calculus of Hilb (Theorem 2.7); the second equality is by naturality of $\alpha$. We therefore indeed have a map $Z \rightarrow B(H)$, which we now show is a $*$-homomorphism.

- Multiplicative. Recalling the definition of the multiplication (45) of $Z$, we show $\pi_{\alpha}([V, v] \cdot[W, w])=$ $\pi_{\alpha}(v) \pi_{\alpha}(w)$ :
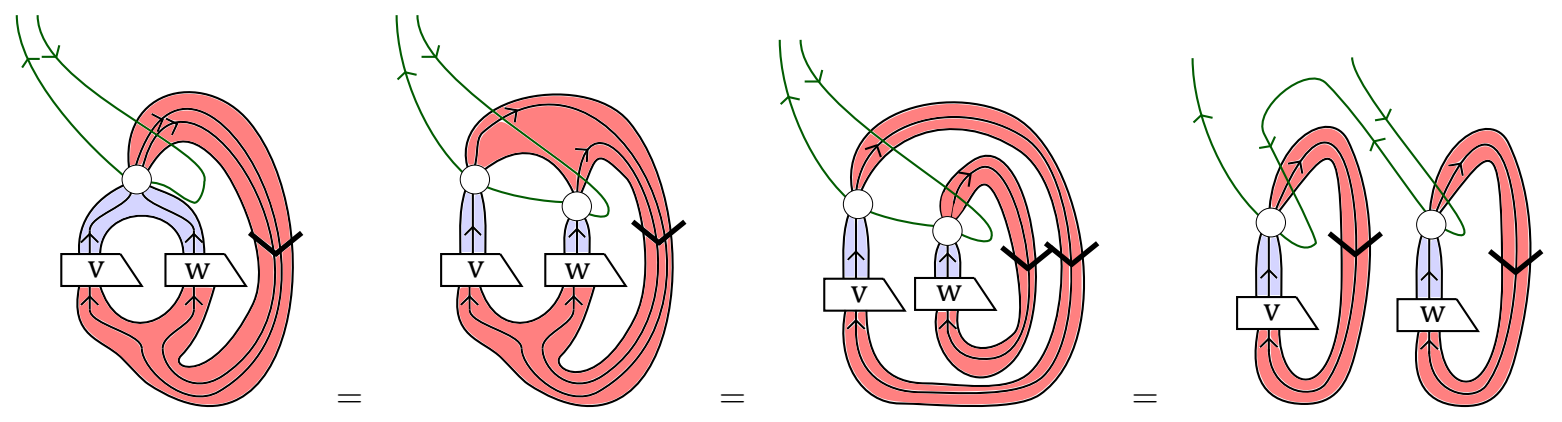

(51)

Here the first equality is by monoidality of $\alpha$, and for the second we slide the multiplicator $F_{2}(V \otimes W) \rightarrow F_{2}(V) \otimes F_{2}(W)$ around the loop and use unitarity of $F_{2}$ to cancel it and its inverse. The third equality is by the graphical calculus of the symmetric monoidal category Hilb. In the last diagram we recognise the multiplication of $B(H) \cong H \otimes H^{*}(20)$. 
- Unital. Recalling that the unit of $Z$ is $\left[\mathbb{1}, u_{1} \circ u_{2}^{\dagger}\right]$, we observe:

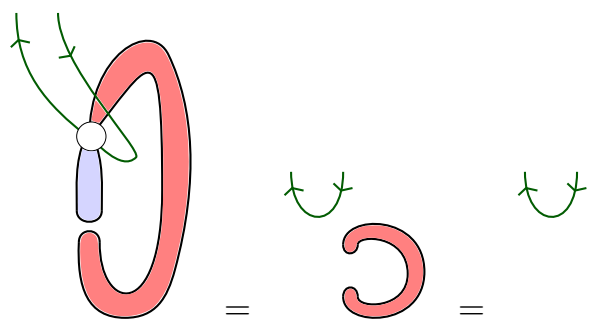

Here the first equality is by monoidality of $\alpha$; for the second we slide the unitor around the loop and use unitarity of $F_{2}$ to cancel it with its inverse. In the final diagram we recognise the unit of $B(H) \cong H \otimes H^{*}(20)$.

- Involution-preserving. Recalling the definition of the involution (47) on generators $[V, v]$, we have the following equations. We use a large double upwards arrow for the dual $F_{2}\left(V^{*}\right)^{*}$ in Hilb, and a large downwards arrow for the dual $F_{2}(V)^{*}$ in Hilb:
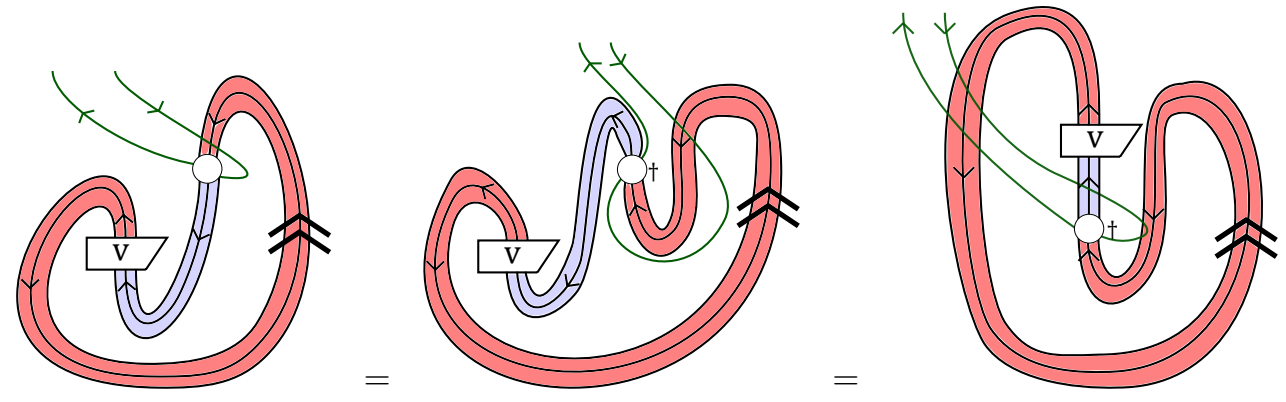

Here the first equality is by unitarity of $\alpha(40)$, and the second equality is by a snake equation for the induced duality on $F_{1}(V)$. We now observe that, since $F_{2}\left(V^{*}\right)$ is dual to $F_{2}(V)$ in Hilb by the induced cup and cap, by Proposition 2.3 there is an invertible morphism $f: F_{2}\left(V^{*}\right)^{*} \rightarrow F_{2}(V)$ relating the two caps:

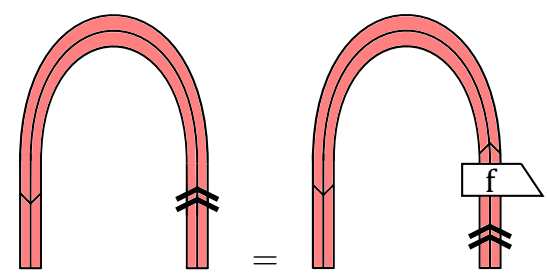

We now continue (53):
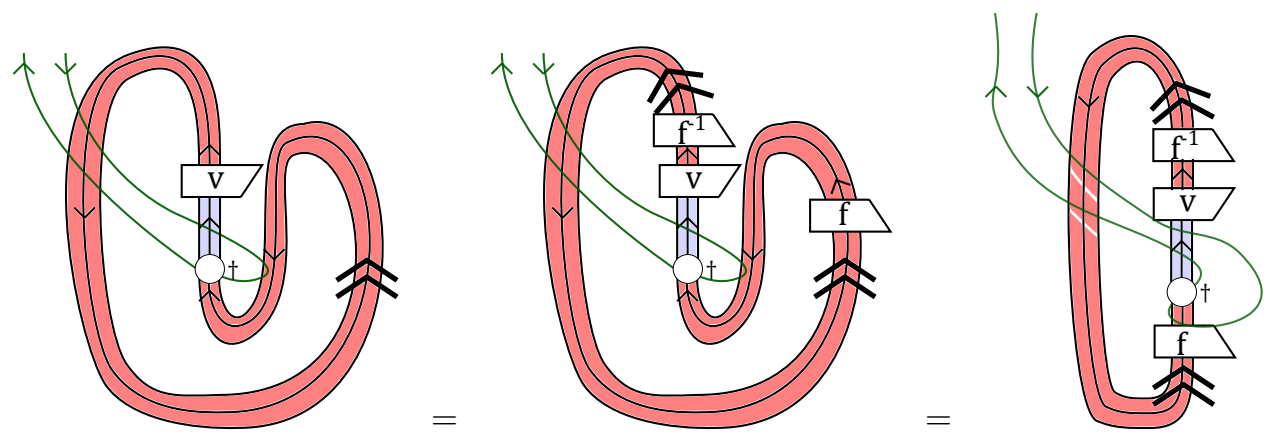


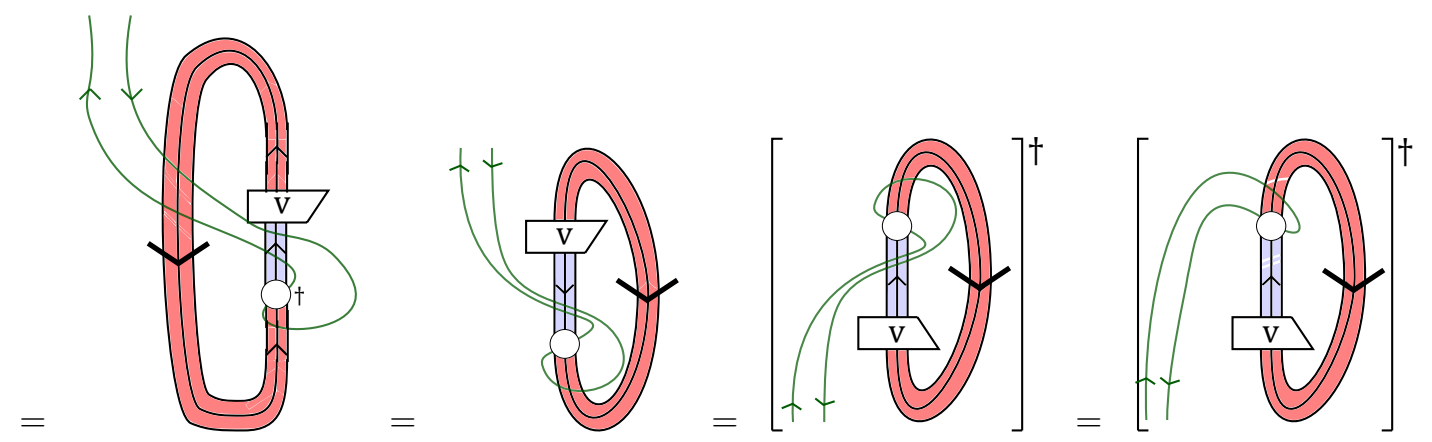

$(56)$

For the first equality we used (54); for the second we used a snake equation for the induced dual on $F_{2}(V)$; for the third we slid $f^{-1}$ around the loop and cancelled it with $f$; for the fourth we used the graphical calculus of Hilb to pull the loop around; the fifth equality is by the 'horizontal reflection' calculus of the dagger in Hilb; and for the sixth equality we used the graphical calculus of Hilb to untangle the $H$-wires. In the final diagram we observe the involution of $B(H)(20)$.

Finally, every modification clearly induces an intertwiner:

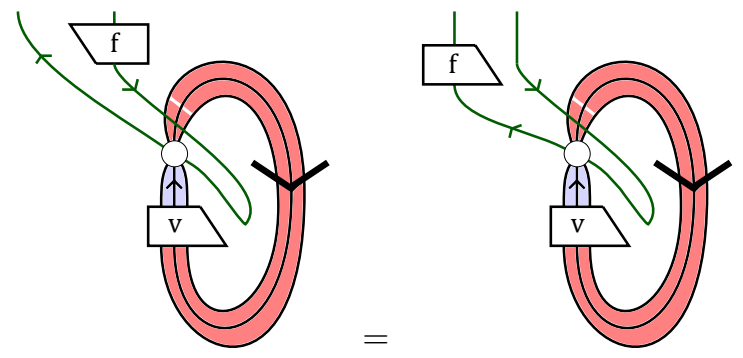

We will now produce a construction in the other direction, which gives a UPT $F_{1} \rightarrow F_{2}$ for any *-representation of $Z$.

Construction 3.10. Let $\pi: Z \rightarrow B(H)$ be a $*$-representation. Recall that $Z \cong \oplus_{V \in \operatorname{Obj}(\mathcal{C})} F_{1}(V) \otimes$ $F_{2}(V)^{*} / \mathcal{N}$. For every object $V$ of $\mathcal{C}$ we define a map $U_{V}: F_{1}(V) \rightarrow F_{2}(V) \otimes Z$ as follows (c.f. [16, Thm 2.3.11]):
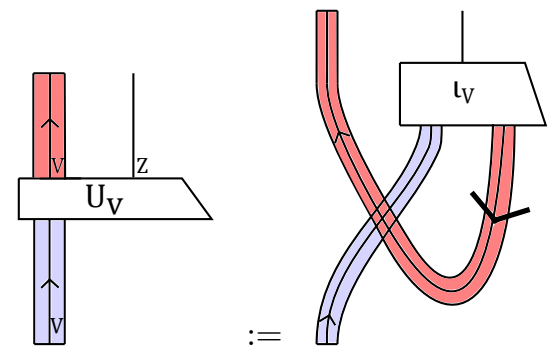

Here $\iota_{V}: F_{1}(V) \otimes F_{2}(V)^{*} \rightarrow Z$ is the canonical map induced by the direct sum.

We then define a map $\left(\alpha_{\pi}\right)_{V}: F_{1}(V) \otimes H \rightarrow H \otimes F_{2}(V)$ (here $\pi: Z \rightarrow B(H) \cong H \otimes H^{*}$ is 
represented by a white vertex):

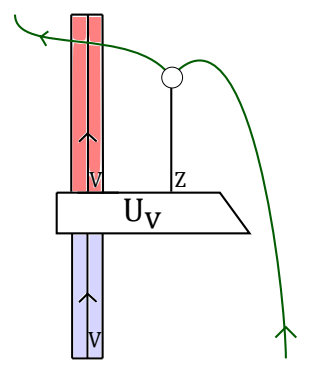

Remark 3.11. The diagrams with a $Z$-wire are to be interpreted in the symmetric monoidal category Vec of possibly infinite-dimensional complex vector spaces, since $Z$ is not generally a finite-dimensional Hilbert space. We use more structure (wire bending, dagger, etc.) only on the parts of the diagram which come from Hilb.

Proposition 3.12. Construction 3.10 defines a UPT $\left(\alpha_{\pi}, H\right): F_{1} \rightarrow F_{2}$. Moreover, any intertwiner $f:(\pi, H) \rightarrow\left(\pi^{\prime}, H^{\prime}\right)$ induces a modification $f:\left(\alpha_{\pi}, H\right) \rightarrow\left(\alpha_{\pi^{\prime}}, H^{\prime}\right)$.

Proof. We show that the map $U_{V}$ satisfies certain properties which will imply that $\alpha_{\pi}$ is a UPT.

- Naturality. For any $f: V \rightarrow W$ in $\mathcal{C}$ :
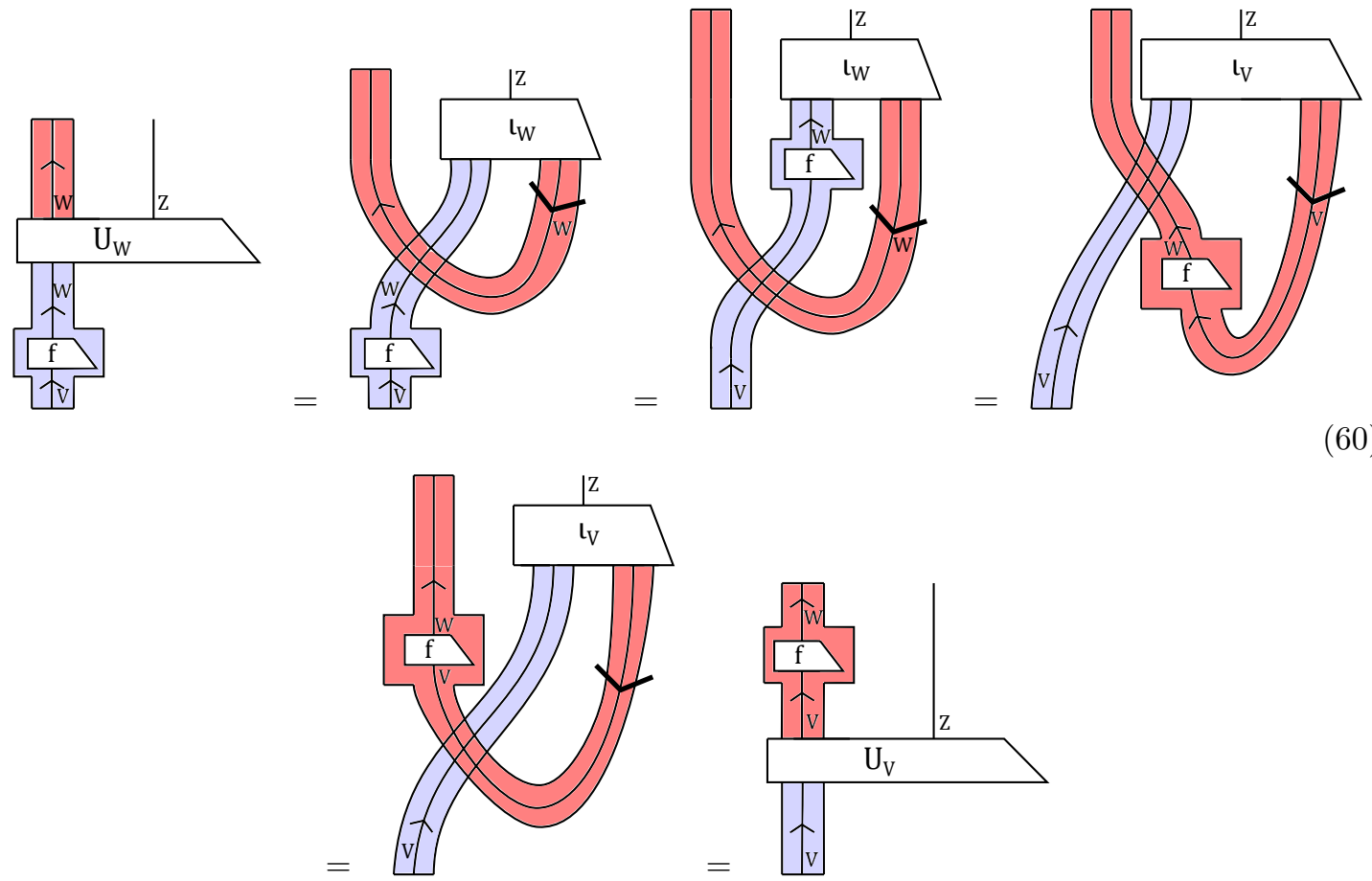

Here the first and fifth equalities are by definition, and the second and fourth equalities are by naturality of the symmetry in Hilb. The third equality follows from the definition of $Z$ as the quotient space $\oplus_{V \in \operatorname{Obj}(\mathcal{C})} F_{1}(V) \otimes F_{2}(V)^{*} / \mathcal{N}$.

- Monoidality. 
- For any object $V$ of $\mathcal{C}$ let $\{|i\rangle\}$ and $\{|j\rangle\}$ be orthonormal bases of $F_{1}(V)$ and $F_{2}(V)$ respectively. Then inserting resolutions of the identity in (58) we obtain the following expression for the map $U_{V}$ :

$$
U_{V}=\sum_{i, j}|j\rangle\langle i| \otimes[V,|i\rangle\langle j|]
$$

We observe that the map $U_{V}$ does not depend on the choice of orthonormal basis, since it was defined in a basis-independent way to begin with. We want to show the following equation:

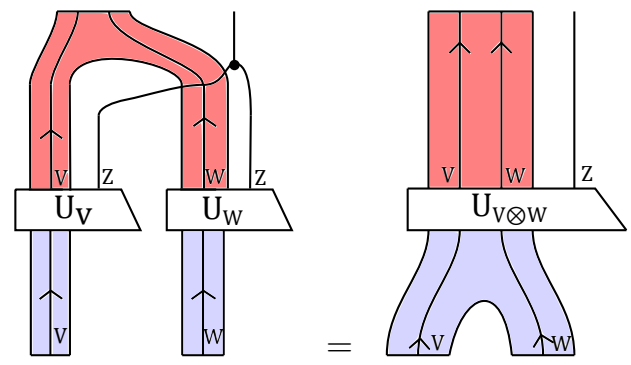

For this we have the following equation, where $\{|i\rangle\},\{|j\rangle\},\{|k\rangle\},\{|l\rangle\}$ are orthonormal bases of $F_{1}(V), F_{2}(V), F_{1}(W)$ and $F_{2}(W)$ respectively:

$$
\begin{array}{r}
\sum_{i, j, k, l}\left(m_{V, W} \circ(|j\rangle\langle i|\otimes| l\rangle\langle k|)\right) \otimes\left[V \otimes W, m_{V, W} \circ(|i\rangle\langle j|\otimes| k\rangle\langle l|) \circ m_{V, W}^{\dagger}\right] \\
=\sum_{i, j, k, l}\left(m_{V, W} \circ(|j\rangle\langle i|\otimes| l\rangle\langle k|) \circ m_{V, W}^{\dagger} \circ m_{V, W}\right) \otimes\left[V \otimes W, m_{V, W} \circ(|i\rangle\langle j|\otimes| k\rangle\langle l|) \circ m_{V, W}^{\dagger}\right]
\end{array}
$$

Looking at the last expression, we observe that it has the form (62) for the orthonormal bases $m_{V, W} \circ(|i\rangle \otimes|k\rangle)$ and $m_{V, W} \circ(|j\rangle \otimes|l\rangle)$ of $F_{1}(V \otimes W)$ and $F_{2}(V \otimes W)$. The equation (63) follows.
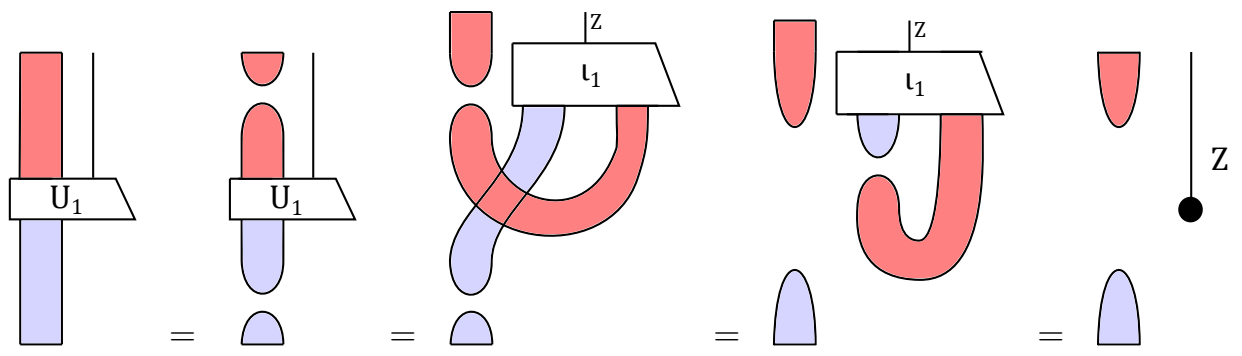

Here the first equality is by unitarity of the unitors of $F, F^{\prime}$; the second equality is by definition of $U_{\mathbb{1}}$; the third equality is by the graphical calculus of Hilb; and the fourth equality is by definition of the unit of $Z$ (which we have drawn as a black vertex).

- Unitarity. Choose orthonormal bases of $F(V), F^{\prime}(V)$. We show that with respect to such a basis $U_{V}$ is a unitary matrix. We prove the first unitarity equation $\sum_{k}\left[U_{V}\right]_{k i}^{*}\left[U_{V}\right]_{k j}=\delta_{i j} 1_{Z}$. Let $\{|i\rangle\},\{|j\rangle\}$ be elements of the orthonormal basis of $F(V)$ and let $\{|k\rangle\}$ be the orthonormal basis 
of $F^{\prime}(V)$. Then:

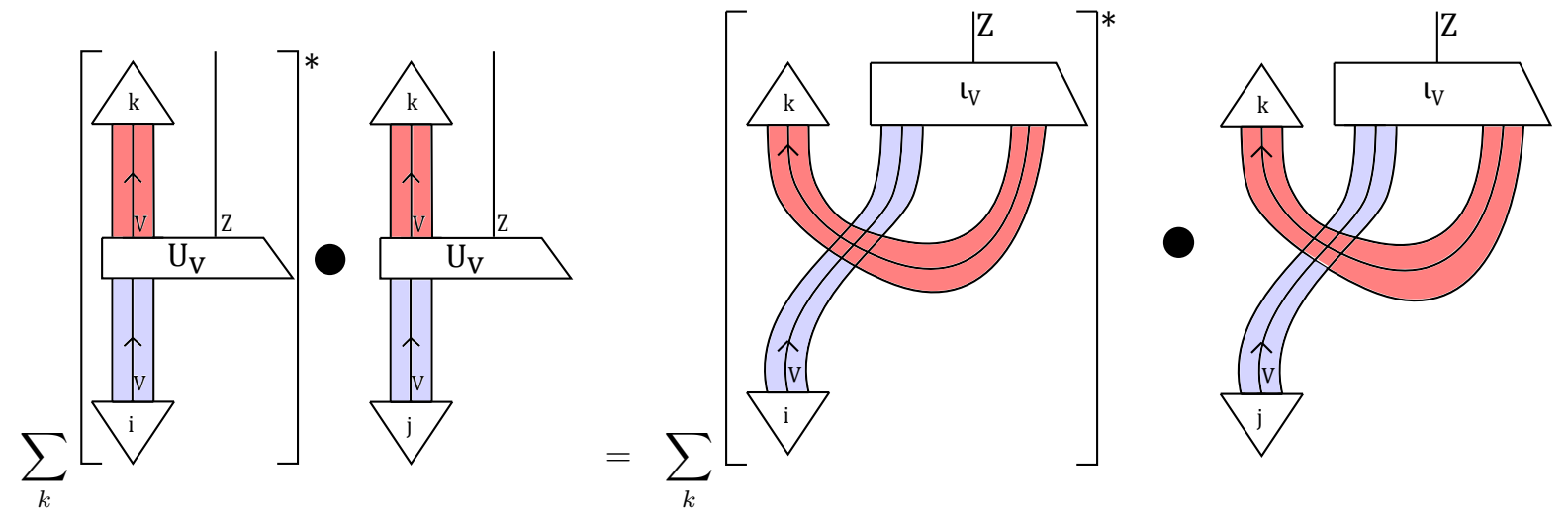

(65)
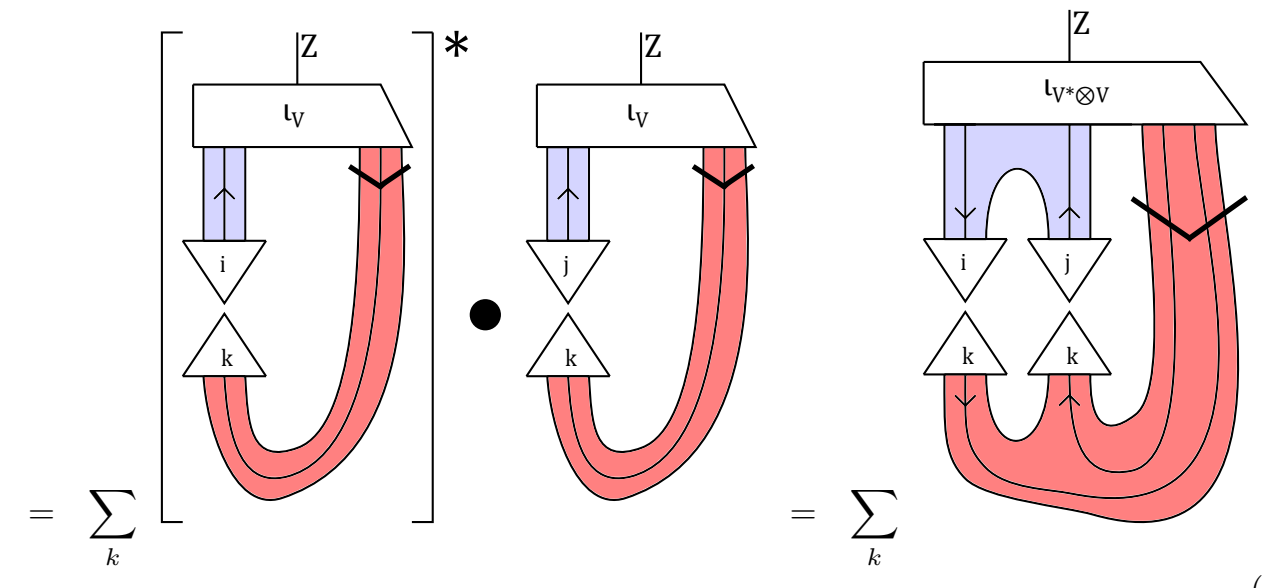

(66)
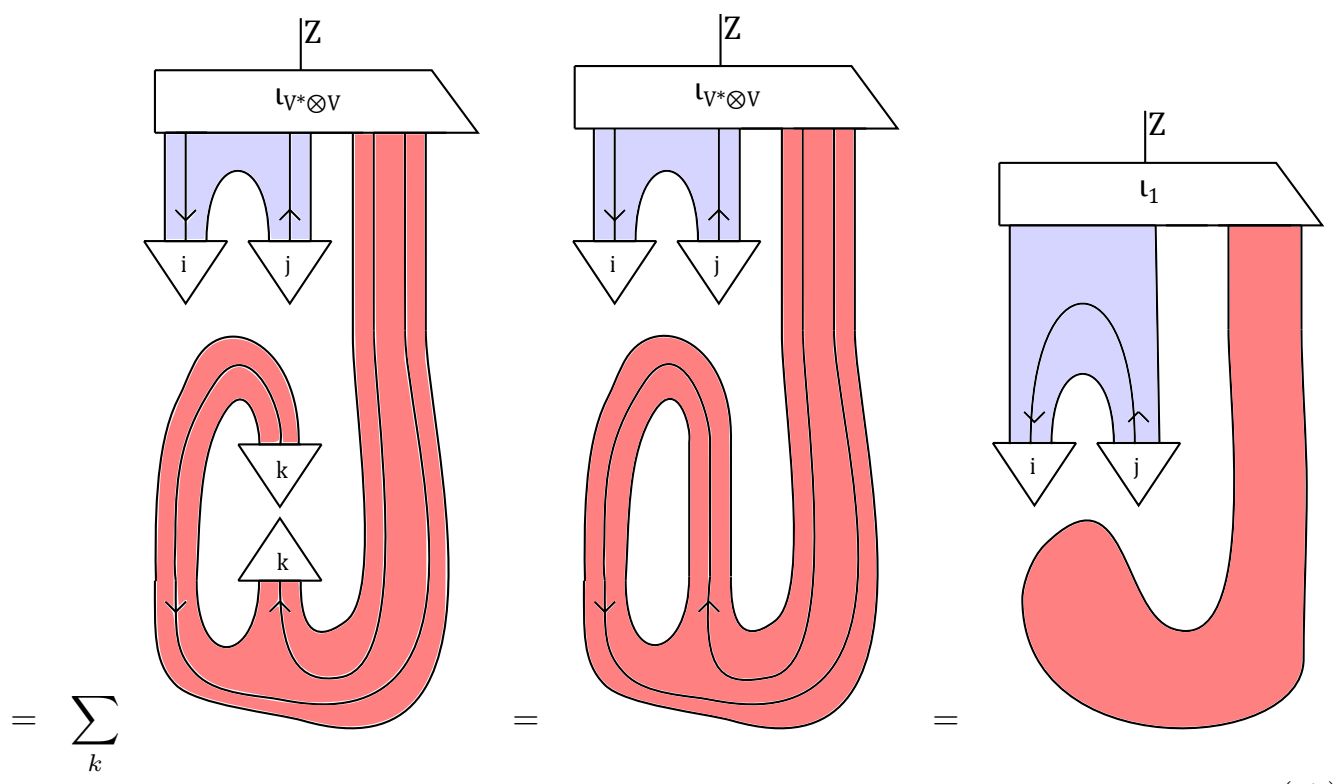

(67) 


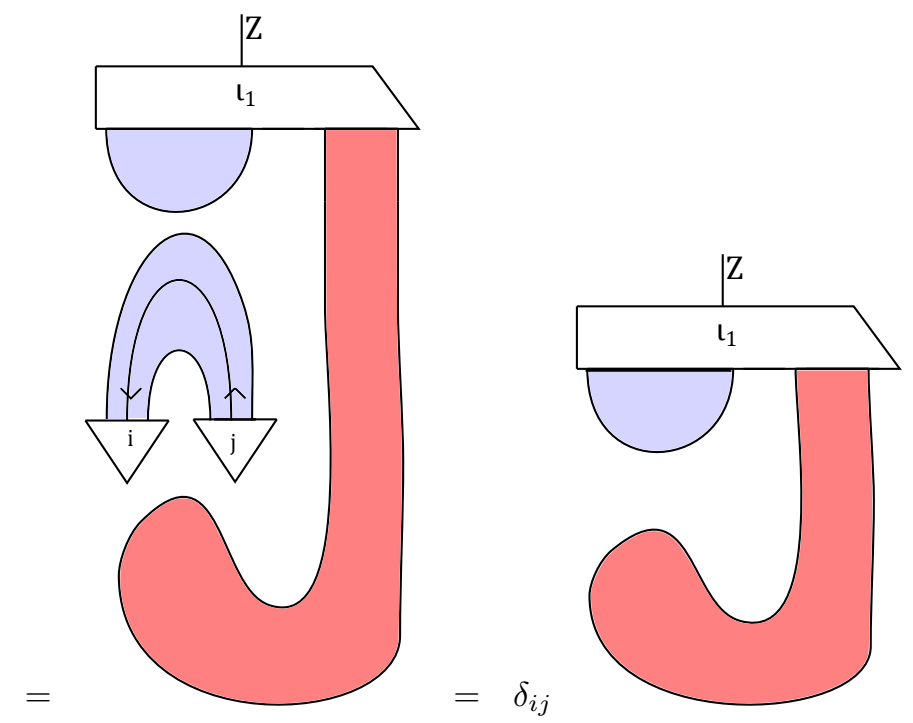

Here for the first equality we used the definition of $U_{V}$; for the second equality we used the graphical calculus of Hilb; for the third equality we used the definition of multiplication and involution in $Z$; for the fourth equality we used the definition of the involution in $Z$; for the fifth equality we removed the resolution of the identity and used unitarity of the functor $F^{\prime}$; for the sixth equality we used the definition of the quotient space defining $Z$ (43); for the seventh equality we used unitarity of the functor $F$; and for the eighth equality we used the induced duality from $\mathcal{C}$ and orthogonality of the basis. In the final diagram we recognise the unit of $Z$.

The second unitarity equation, $\sum_{k}\left[U_{V}\right]_{i k}\left[U_{V}\right]_{j k}^{*}=\delta_{i j} 1_{Z}$ is shown similarly.

We now show that $\alpha_{\pi}$ is a UPT.

- Naturality. Follows immediately from naturality of $\left\{U_{V}\right\}$ and of the swap.

- Monoidality.
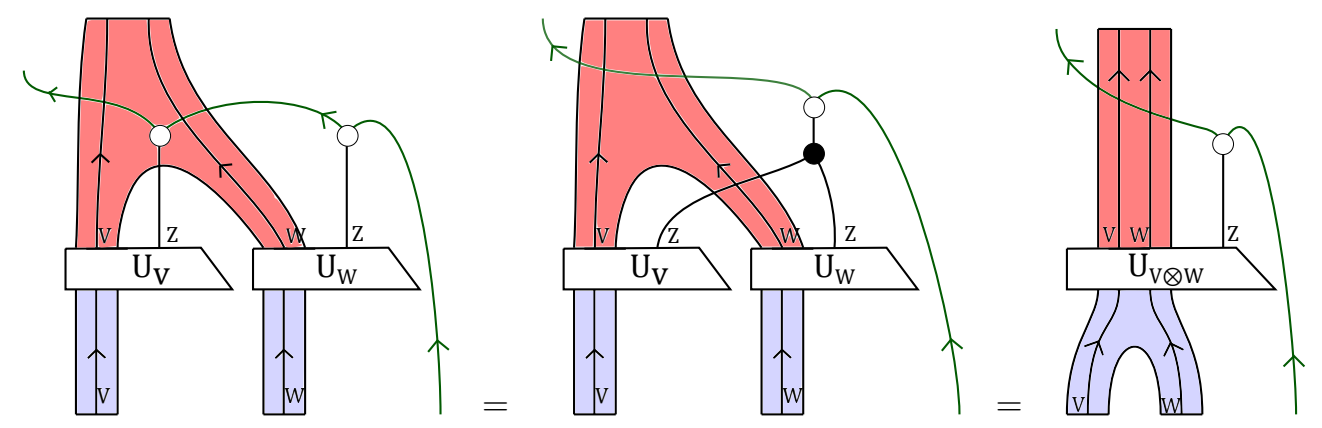

In the first equation we recognised the multiplication of $B(H) \cong H \otimes H^{*}(20)$ and used the fact that $\pi$ is a homomorphism. In the second equation we used monoidality of $U$.

- Unitarity. We must show that $\alpha_{V}^{\dagger}$ is a 2-sided inverse for $\alpha_{V}$. Consider the first of the 2 
unitarity equations:

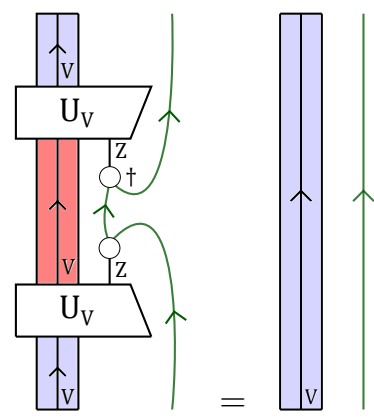

Remark 3.13. As was already stated in Remark 3.11, there is not necessarily a Hilbert space structure on $Z$; however, in (70) the dagger of $\pi: Z \rightarrow B(H)$ appears. This is just a convenient way of representing the dagger of the morphism $\left(\operatorname{id}_{F_{2}(V)} \otimes \pi\right) \circ U_{V}$ and should only be interpreted in that context.

To prove (70), we choose orthonormal bases for $F_{1}(V)$ and $F_{2}(V)$. Let $|i\rangle,|j\rangle$ be any two elements of the orthonormal basis for $F_{1}(V)$ and let $\{|k\rangle\}$ be the orthonormal basis elements of $F_{2}(V)$. Then we have the following equation:

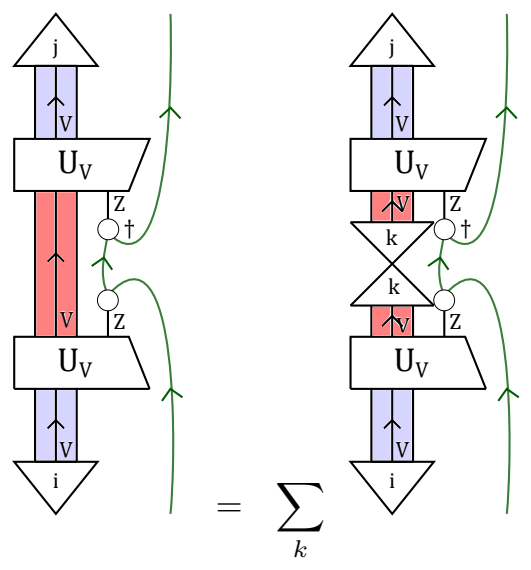

Here we simply inserted a resolution of the identity in $F_{2}(V)$. Now we define the following elements of $B(H)$ :

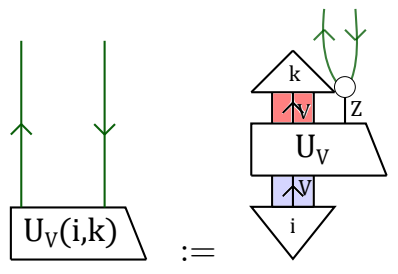


With these elements (71) becomes:

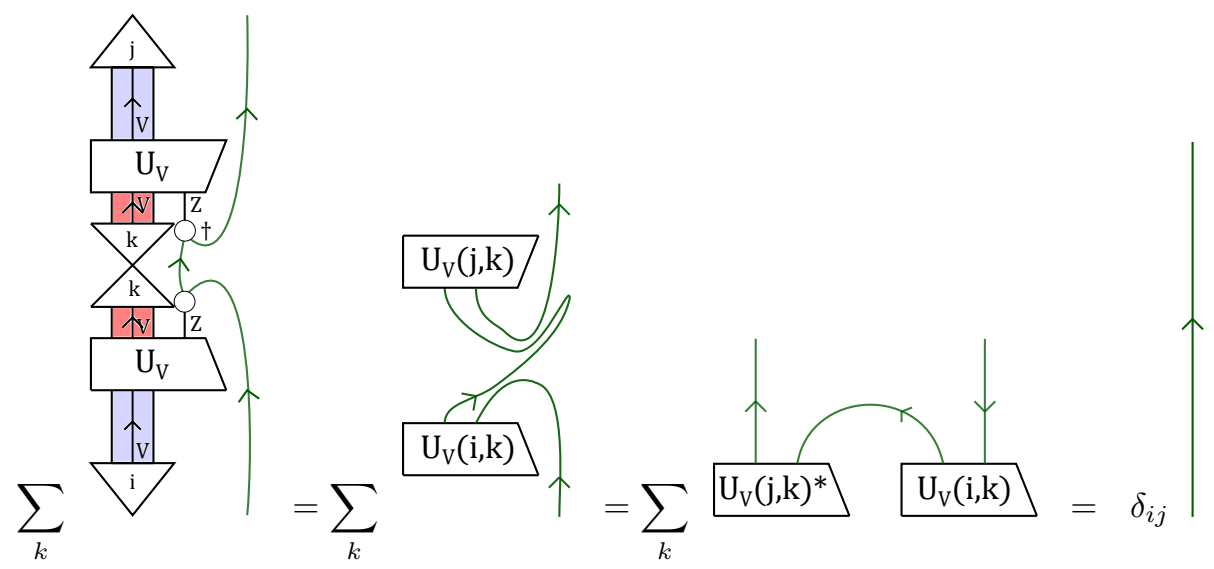

Here in the second equality we noticed the involution and multiplication of $B(H) \cong H \otimes H^{*}(20)$.

For the third equality we used involutivity and multiplicativity of $\pi$, and then unitarity of $U_{V}$. Therefore (70) is proved. The proof of the other unitarity equation is similar.

Finally, an intertwiner clearly induces a modification:
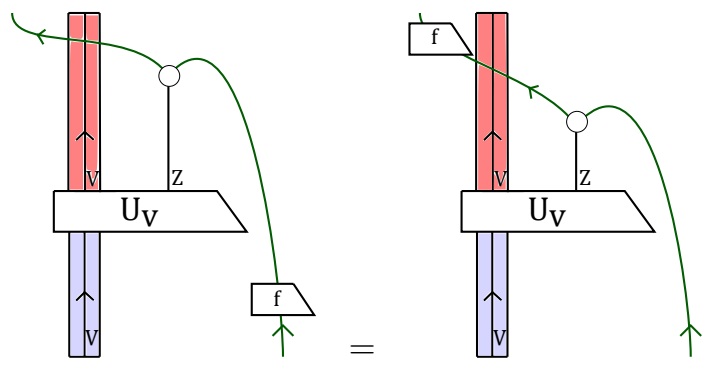

Theorem 3.14. Let $\mathcal{C}$ be a $C^{*}$-tensor category with conjugates, and let $F_{1}, F_{2}: \mathcal{C} \rightarrow$ Hilb be fibre functors. Let $Z=H_{o m} \vee\left(F_{1}, F_{2}\right)$ be the corresponding $A_{G_{2}}-A_{G_{1}}$-bi-Hopf-Galois-object. There is an isomorphism of categories between:

- The category $\operatorname{Rep}(Z)$ of f.d. *-representations of $Z$ and intertwiners.

- The category $\operatorname{Fun}\left(F_{1}, F_{2}\right)$ of UPTs $F_{1} \rightarrow F_{2}$ and modifications.

Proof. We show that Constructions 3.8 and 3.10 are strictly inverse. First we observe that, for a UPT $\alpha: F_{1} \rightarrow F_{2}$, the $*$-homomorphism $\pi_{\alpha}: Z \rightarrow B(H)$ is defined on the subspace in the image of $\iota_{V}: F_{1}(V) \otimes F_{2}(V)^{*} \rightarrow Z$ as follows:

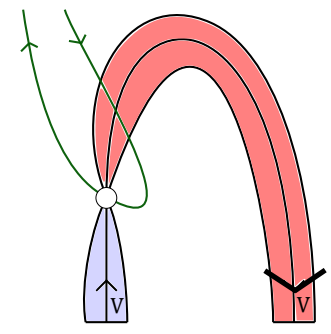


We also observe that, for a $*$-homomorphism $\pi: Z \rightarrow B(H)$, the UPT $\alpha_{\pi}: F_{1} \rightarrow F_{2}$ is defined on $V$ as follows:

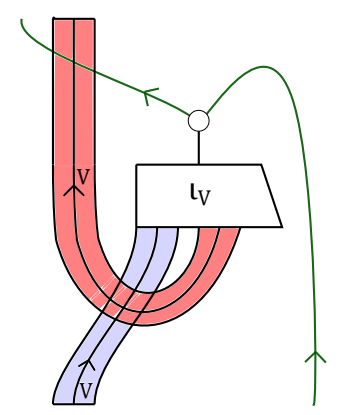

For one direction, let $\alpha: F_{1} \rightarrow F_{2}$ be a UPT and use Construction (3.8) to obtain a $*$-representation $\pi_{\alpha}: Z \rightarrow B(H)$. Now use Construction 3.10 to obtain a UPT $F_{1} \rightarrow F_{2}$. That the resulting UPT is $\alpha$ follows immediately from the graphical calculus of Hilb:

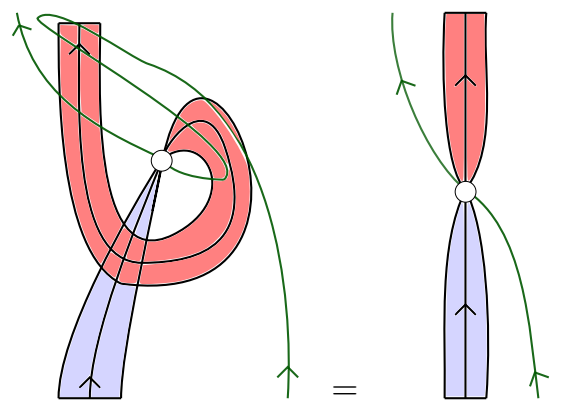

In the other direction, let $\pi$ be a $*$-representation $\pi_{\alpha}: Z \rightarrow B(H)$ and use Construction (3.10) to obtain a UPT $\alpha_{\pi}: F_{1} \rightarrow F_{2}$. Now use Construction 3.8 to obtain a $*$-representation $Z \rightarrow B(H)$. That the resulting UPT is $\alpha$ again follows immediately from the graphical calculus of Hilb:

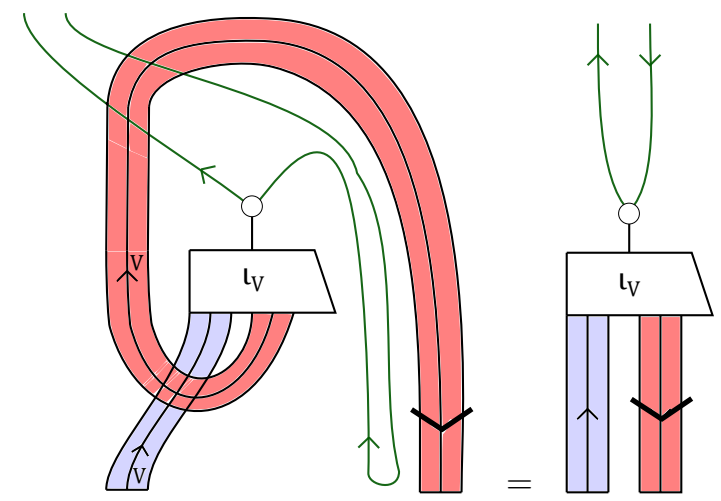

That the maps on modifications and intertwiners are inverse is clear.

Corollary 3.15. Two fibre functors $F_{1}, F_{2}$ on $\operatorname{Rep}(G)$ are related by a UPT $F_{1} \rightarrow F_{2}$ precisely when the corresponding $A_{G_{2}}-A_{G_{1}}$-bi-Hopf-Galois object $\operatorname{Hom}^{\vee}\left(F_{1}, F_{2}\right)$ has a finite-dimensional $*$-representation.

Definition 3.16. Let $\mathcal{C}$ be a semisimple pivotal dagger category and let $F: \mathcal{C} \rightarrow$ Hilb be a fibre functor. We say that a fibre functor $F^{\prime}: \mathcal{C} \rightarrow$ Hilb is accessible from $F$ if there exists a UPT $\alpha: F \rightarrow F^{\prime}$.

Let $G$ be a compact quantum group and $F: \operatorname{Rep}(G) \rightarrow$ Hilb the canonical fibre functor. We observe that a UPT - since its components are unitary - must preserve dimensions of Hilbert spaces in the 
sense that $\operatorname{dim}(F(V))=\operatorname{dim}\left(F^{\prime}(V)\right)$, so an $A_{G}$-Hopf-Galois object corresponding to a fibre functor accessible from $F$ must be cleft [4, Thm 1.17]. It is unknown to the author whether all cleft Hopf-Galois objects admit a finite-dimensional $*$-representation.

At least in one case the situation is clear, since cleft Hopf-Galois objects for a compact quantum group algebra $A$ are all obtained as cocycle twists of $A[4$, Thm 1.8], so for finite-dimensional $A$ they are also finite-dimensional with a faithful state [4, Prop. 4.2.5, Cor. 4.3.5].

Corollary 3.17. When $G$ is a finite $C Q G$ (i.e the algebra $A_{G}$ is finite-dimensional) all fibre functors on $\operatorname{Rep}(G)$ are accessible from the canonical fibre functor by a UPT.

\section{Morita theory of accessible fibre functors and UPTs}

In Section 3.2 we showed that a fibre functor on the category $\operatorname{Rep}(G)$ is accessible from the canonical fibre functor $F$ by a UPT precisely when the corresponding $A_{G}$-Hopf-Galois object admits a finitedimensional $*$-representation. In this Section we will use Morita theory to show that accesible fibre functors $F^{\prime}$ and UPTs $F \rightarrow F^{\prime}$ can be classified in terms of the finite-dimensional representation theory of the compact quantum group algebra $A_{G}$.

\subsection{Background on Frobenius monoids and Morita equivalence}

Morita theory relates 1-morphisms $X: r \rightarrow s$ out of an object $r$ of a 2-category $\mathcal{C}$ to Frobenius monoids in its category of endomorphisms $\mathcal{C}(r, r)$. In our case, the 2 -category in question is $\operatorname{Fun}(\operatorname{Rep}(G), \operatorname{Hilb})$, and we consider the category $\operatorname{End}(F)$ of endomorphisms of the canonical fibre functor $F: \operatorname{Rep}(G) \rightarrow$ Hilb, which we have just shown (Theorem 3.14) is isomorphic to $\operatorname{Rep}\left(A_{G}\right)$. We now recall the definition of a Frobenius monoid, and two notions of equivalence which will be important in our classification.

\section{Frobenius monoids.}

Definition 4.1. Let $\mathcal{C}$ be a monoidal dagger category. A monoid in $\mathcal{C}$ is an object $A$ with multiplication and a unit morphisms, depicted as follows:

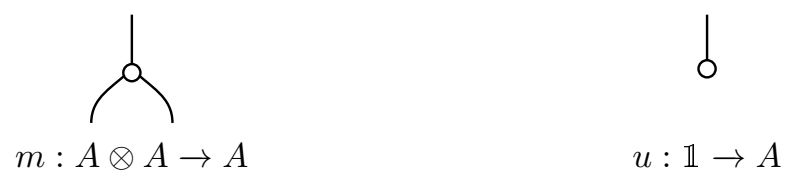

These morphisms satisfy the following associativity and unitality equations:

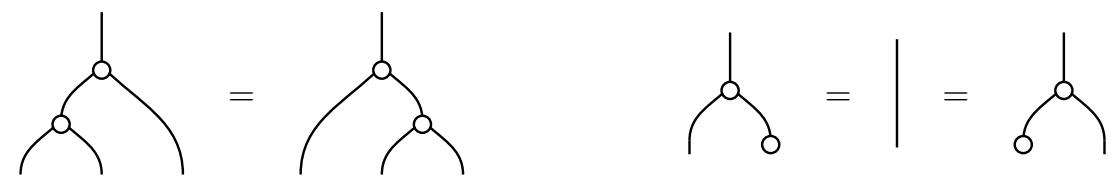

Analogously, a comonoid is an object $A$ with a coassociative comultiplication $\delta: A \rightarrow A \otimes A$ and a counit $\epsilon: A \rightarrow \mathbb{C}$. The dagger of an monoid $(A, m, u)$ is a comonoid $\left(A, m^{\dagger}, u^{\dagger}\right)$. A monoid $(A, m, u)$ in $\mathcal{C}$ is called Frobenius if the monoid and adjoint comonoid structures are related by the following Frobenius equation:<smiles>CCOC(C)C(C)OC(C)OC(C)OC</smiles> 
Frobenius monoids are canonically self-dual. Indeed, it is easy to see that for any Frobenius monoid the following cup and cap fulfil the snake equations (5):

$$
r:=\gamma_{0}^{0}:=\underbrace{}_{0}
$$

A Frobenius monoid is special if the following additional equation is satisfied:

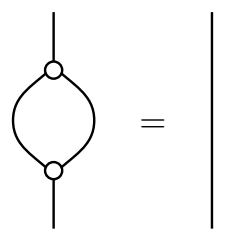

Example 4.2. The following normalisation of the endomorphism algebra of Definition 2.15 is a special Frobenius monoid on $H \otimes H^{*}$, where $d$ is the dimension of $H$ :
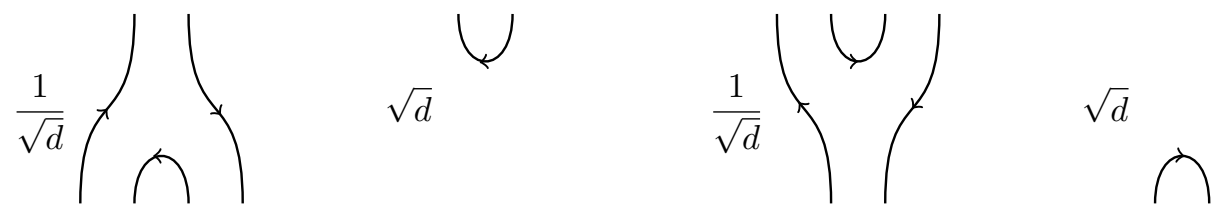

We now consider two equivalence relations on special Frobenius monoids in a dagger category with split idempotents. The stricter of the relations is unitary $*$-isomorphism.

\section{Unitary $*$-isomorphism.}

Definition 4.3. Let $A, B$ be Frobenius monoids in a monoidal dagger category. We say that a morphism $f: A \rightarrow B$ is a $*$-homomorphism precisely when it satisfies the following equations (we use black vertices for the Frobenius monoid $B$ ):
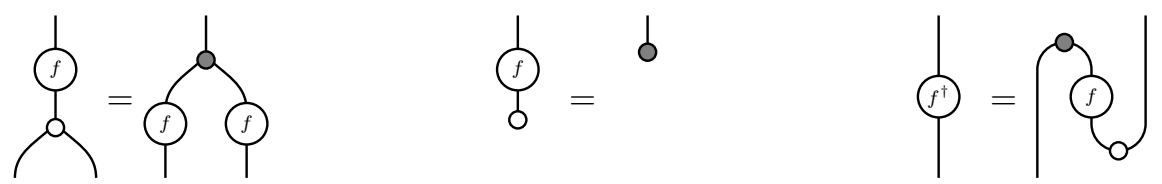

We call a unitary $*$-homomorphism a unitary $*$-isomorphism. It is easy to see that a unitary $*$ isomorphism satisfies the following additional equations:
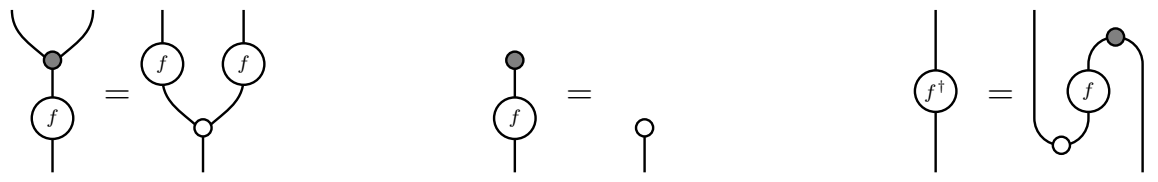

Morita equivalence. To define the weaker equivalence relation, we introduce the notion of a dagger bimodule.

Definition 4.4. Let $A$ and $B$ be special Frobenius monoids in a monoidal dagger category. An $A-B$ dagger bimodule is an object $M$ together with an morphism $\rho: A \otimes M \otimes B \rightarrow M$ fulfilling the following equations:
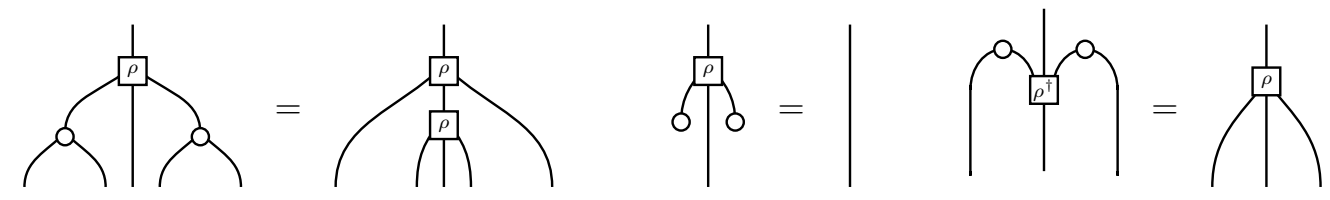
We usually denote an $A$-B-dagger bimodule $M$ by ${ }_{A} M_{B}$.

Definition 4.5. A morphism of dagger bimodules ${ }_{A} M_{B} \rightarrow{ }_{A} N_{B}$ is a morphism $f: M \rightarrow N$ that commutes with the action of the Frobenius monoids:

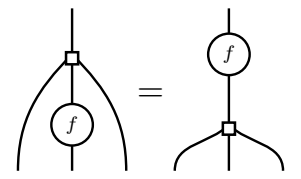

Two dagger bimodules are isomorphic, here written ${ }_{A} M_{B} \cong{ }_{A} N_{B}$, if there is a unitary morphism of dagger bimodules ${ }_{A} M_{B} \rightarrow{ }_{A} N_{B}$.

In a monoidal dagger category in which dagger idempotents split (Definition 2.21), we can compose dagger bimodules ${ }_{A} M_{B}$ and ${ }_{B} N_{C}$ to obtain an $A-C$-dagger bimodule ${ }_{A} M \otimes_{B} N_{C}$ as follows. First we observe that the following endomorphism is a dagger idempotent:

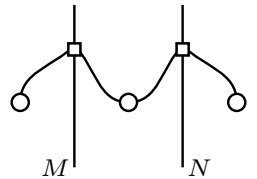

This may be seen as follows. For idempotency:
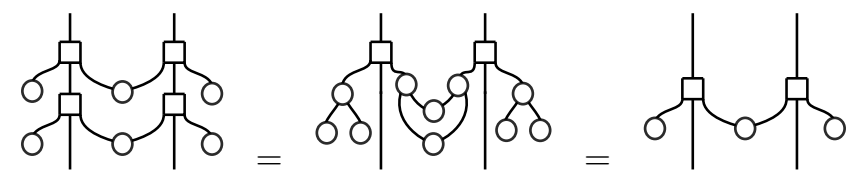

Here for the first equality we used (87) and for the second we used speciality of $B$. For self-adjointness:
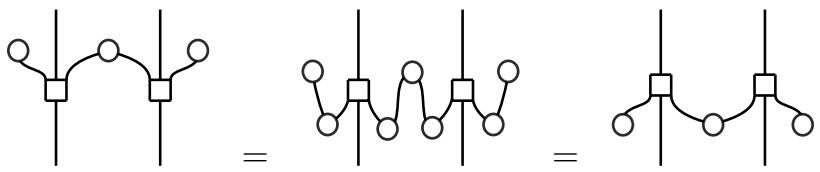

Here for the first equality we used the third equation of (87), and for the second equality we used the axioms of a Frobenius algebra.

Now the relative tensor product ${ }_{A} M \otimes_{B} N_{C}$ is defined as the splitting of the dagger idempotent (89). We depict the isometry $i: M \otimes_{B} N \rightarrow M \otimes N$ as a downwards pointing triangle:
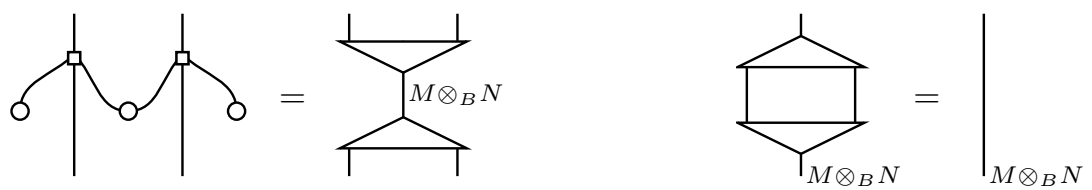

For dagger bimodules ${ }_{A} M_{B}$ and ${ }_{B} N_{C}$, the relative tensor product $M \otimes_{B} N$ is itself an $A-C$-dagger bimodule with the following action $A \otimes\left(M \otimes_{B} N\right) \otimes C \rightarrow M \otimes_{B} N$ :

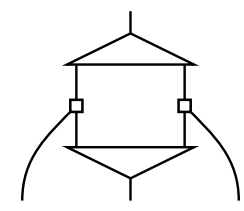

Definition 4.6. Two Frobenius monoids $A$ and $B$ are Morita equivalent if there are dagger bimodules ${ }_{A} M_{B}$ and ${ }_{B} N_{A}$ such that ${ }_{A} M \otimes_{B} N_{A} \cong{ }_{A} A_{A}$ and ${ }_{B} N \otimes_{A} M_{B} \cong{ }_{B} B_{B}$.

It may straightforwardly be verified that unitarily $*$-isomorphic Frobenius monoids are Morita equivalent. 


\subsection{Morita classification of accessible fibre functors and UPTs}

We first observe that every UPT out of the canonical fibre functor $F$ gives rise to a special Frobenius monoid in $\operatorname{End}(F)$.

Proposition 4.7. Let $G$ be a compact quantum group, let $F: \operatorname{Rep}(G) \rightarrow$ Hilb be the canonical fibre functor, and let $F^{\prime}$ be another fibre functor and $(\alpha, H): F \rightarrow F^{\prime}$ a UPT. Then the object $\alpha^{*} \circ \alpha$ of $\operatorname{End}(F)$ has the structure of a special Frobenius monoid in $\operatorname{End}(F)$ with the following multiplication and unit modifications, where $d=\operatorname{dim}(H)$ :

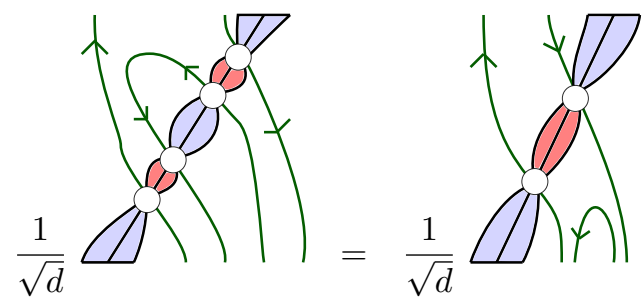

$m:\left(\alpha \otimes \alpha^{*}\right) \otimes\left(\alpha \otimes \alpha^{*}\right) \rightarrow\left(\alpha \otimes \alpha^{*}\right)$

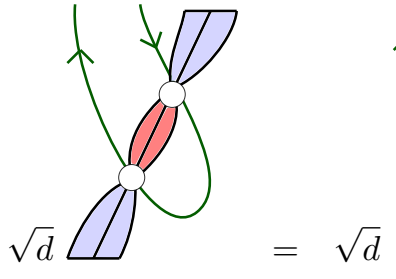

$u: \operatorname{id}_{F} \rightarrow\left(\alpha \otimes \alpha^{*}\right)$

Proof. That these are modifications as claimed follows from the pull-through equations for the cup and cap for the dual UPT (41). It is a special Frobenius monoid because the underlying algebra of the modifications is the normalised endomorphism algebra (Example 4.2).

Definition 4.8. The monoidal functor For : $\operatorname{End}(F) \rightarrow$ Hilb is defined as follows:

- On objects: Every UPT $(\alpha, H): F \rightarrow F$ is taken to its underlying Hilbert space $H$.

- On morphisms: Every modification $f:\left(\alpha_{1}, H_{1}\right) \rightarrow\left(\alpha_{2}, H_{2}\right)$ is taken to its underlying linear map $f: H_{1} \rightarrow H_{2}$.

Remark 4.9. By the isomorphism $\operatorname{End}(F) \cong \operatorname{Rep}\left(A_{G}\right)$ of Theorem 3.14, For is precisely the canonical fibre functor on the category $\operatorname{Rep}\left(A_{G}\right)$.

Definition 4.10. A Frobenius monoid $A=((\alpha, H), m, u)$ in $\operatorname{End}(F)$ is simple if $\operatorname{For}(A)$ is unitarily *-isomorphic to a normalised endomorphism algebra in Hilb.

Every simple Frobenius monoid is in particular special, since it is $*$-isomorphic to a special Frobenius monoid.

Every fibre functor $F^{\prime}$ and UPT $F \rightarrow F^{\prime}$ gives rise to a simple Frobenius monoid in $\operatorname{End}(F)$ by the construction of Proposition 4.7. We now give a construction in the other direction - any simple Frobenius monoid in $\operatorname{End}(F) \cong \operatorname{Rep}\left(A_{G}\right)$ can be 'split' to obtain a fibre functor $F^{\prime}$ and a UPT $F \rightarrow F$ '. First observe that we may conjugate any simple Frobenius monoid $((\tilde{\alpha}, \tilde{H}), \tilde{m}, \tilde{u})$ in $\operatorname{End}(F)$ by the *-isomorphism of Definition 4.10 to obtain a simple Frobenius monoid $\left(\left(\alpha, H \otimes H^{*}\right), m, u\right)$ where the modifications $m, u$ have the standard form:

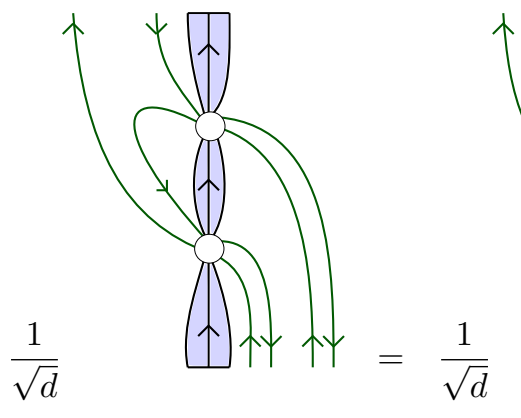

$m: \alpha \circ \alpha \rightarrow \alpha$

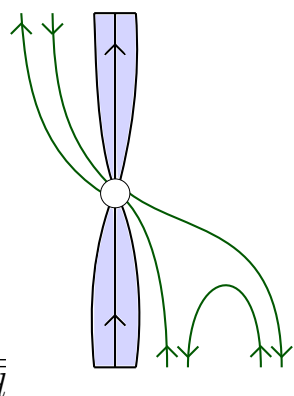

$\sqrt{d}$

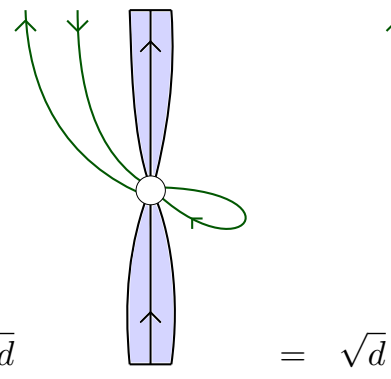

$u: \operatorname{id}_{F} \rightarrow \alpha$

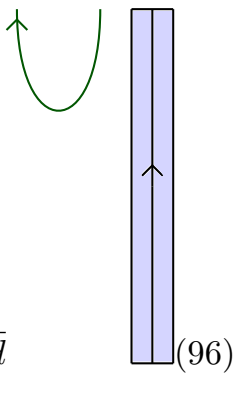

(97) 
Lemma 4.11. For a simple Frobenius monoid $\left(\left(\alpha, H \otimes H^{*}\right), m, u\right)$ in $\operatorname{End}(F)$ and any object $V$ of $\operatorname{Rep}(G)$, the following defines a dagger idempotent on $H^{*} \otimes F(V) \otimes H$ :

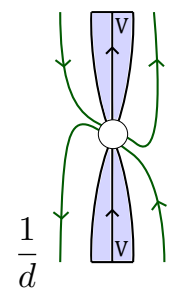

Proof. Idempotency follows from the fact that the algebra multiplication is a modification:

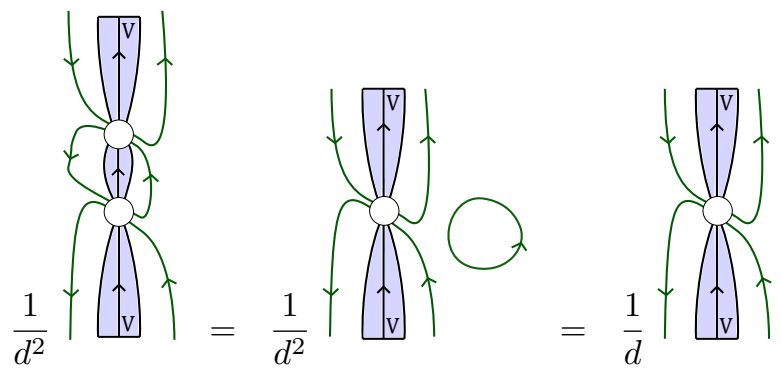

We must now show that the idempotent is self-adjoint. Recall that a Frobenius algebra has a selfduality with the cup and cap (82). By Proposition 2.3 applied in $\operatorname{End}(F)$, there is therefore an invertible modification $P:\left(\alpha, H \otimes H^{*}\right) \rightarrow\left(\alpha^{*},\left(H \otimes H^{*}\right)^{*}\right)$ satisfying the following equations in Hilb:
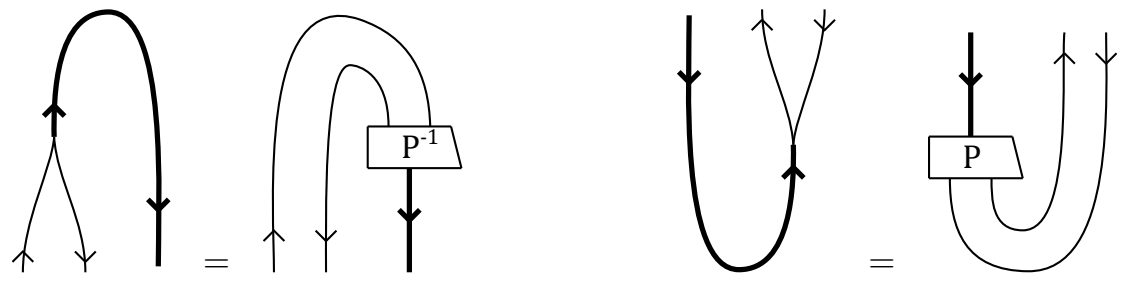

Here we drew the chosen right dual of $\left(H \otimes H^{*}\right)$ using a thick wire and a thick downward arrow in the spirit of (29). Now we show that the idempotent is self-adjoint:

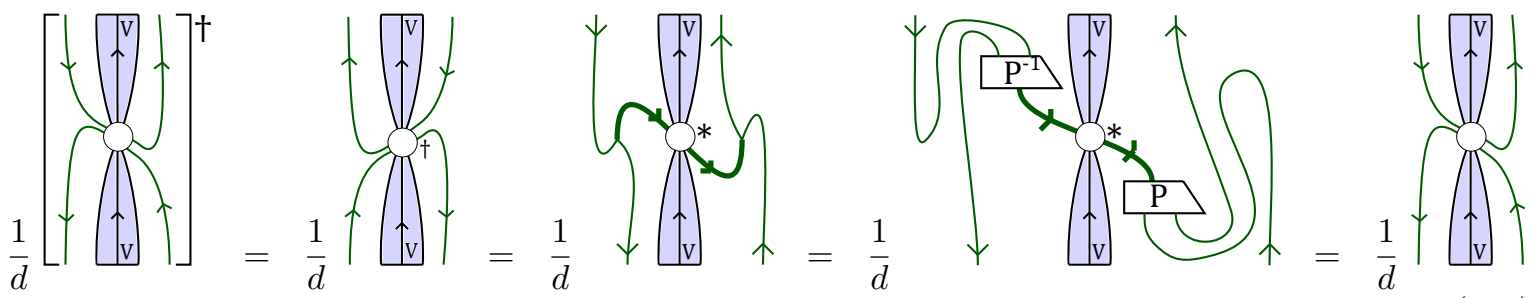

Here for the first equality we used the graphical calculus of the dagger; for the second equality we used unitarity of $\alpha$ (40); for the third equality we used (100); and for the fourth equality we used the fact that $P$ is a modification $\alpha \rightarrow \alpha^{*}$ to cancel $P$ with its inverse.

This dagger idempotent splits to give a new Hilbert space, which, foreshadowing Theorem 4.12, we call $F_{\alpha}(V)$ and draw as $V$ surrounded by a red box, and an isometry $\iota_{V}: F_{\alpha}(V) \rightarrow H^{*} \otimes F(V) \otimes H$ 
satisfying the following equations:
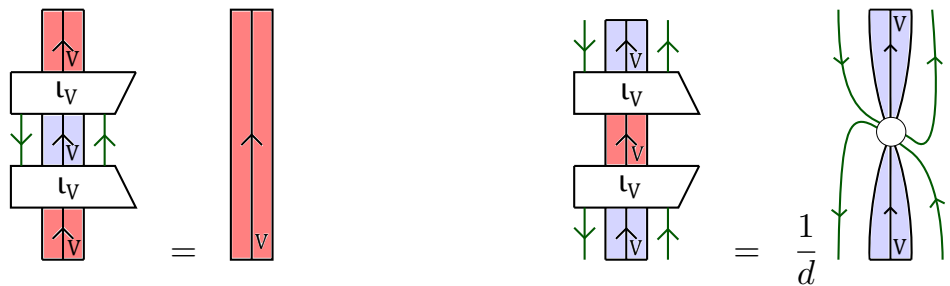

Theorem 4.12. Let $\left(\left(\alpha, H \otimes H^{*}\right), m, u\right)$ be a simple Frobenius monoid in $\operatorname{End}(F) \cong \operatorname{Rep}\left(A_{G}\right)$, and let $d=\operatorname{dim}(H)$. For every representation $V$ of $G$, let $F_{\alpha}(V)$ and $\iota_{V}: F_{\alpha}(V) \rightarrow H^{*} \otimes V \otimes H$ be the Hilbert space and isometry defined in the foregoing discussion.

Then the following defines a fibre functor $F_{\alpha}: \operatorname{Rep}(G) \rightarrow$ Hilb:

- On objects: $V \mapsto F_{\alpha}(V)$.

- On morphisms:

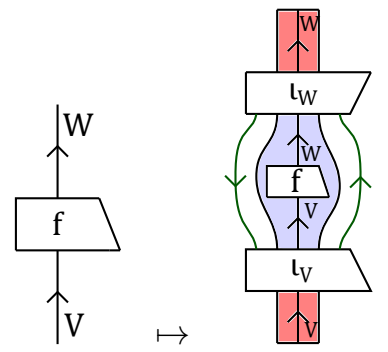

- Monoidal structure:

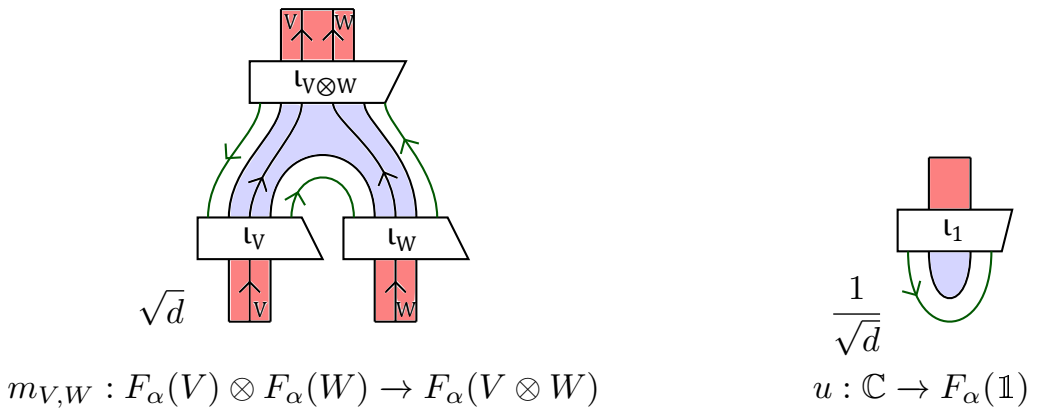

Moreover, there is a UPT $(\sqrt{\alpha}, H): F \rightarrow F_{\alpha}$ with the following components $\sqrt{\alpha}_{V}$ :

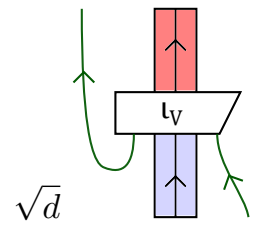

This UPT 'splits' $A$ in the sense that $\sqrt{\alpha}^{*} \circ \sqrt{\alpha}=\alpha$. 
Proof. We first show that $F_{\alpha}$ is a fibre functor. Compositionality is seen as follows:

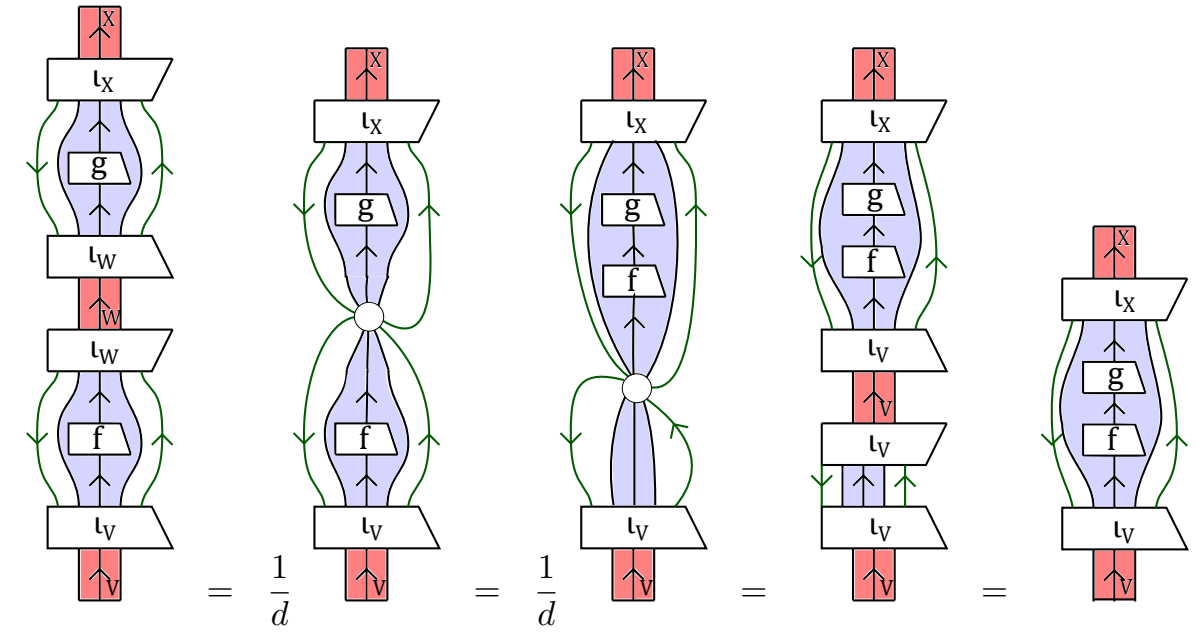

Here the first equality is by the second equation of (102); the second equality is naturality of the UPT $\left(\alpha, H \otimes H^{*}\right): F \rightarrow F$; the third equality is by the second equation of (102); and the fourth equality is by the first equation of (102).

$G$ clearly takes identity morphisms to identity morphisms since $\iota$ is an isometry, and the functor preserves the dagger by symmetry of (103) in a horizontal axis. We therefore already have a unitary functor. For monoidality, we must check that $\left\{m_{V, W}\right\}$ and $u$ are unitary and that they obey the associativity and unitality equations (27) and (28).

- Unitarity of $\left\{m_{V, W}\right\}$. We prove the first equation of unitarity:
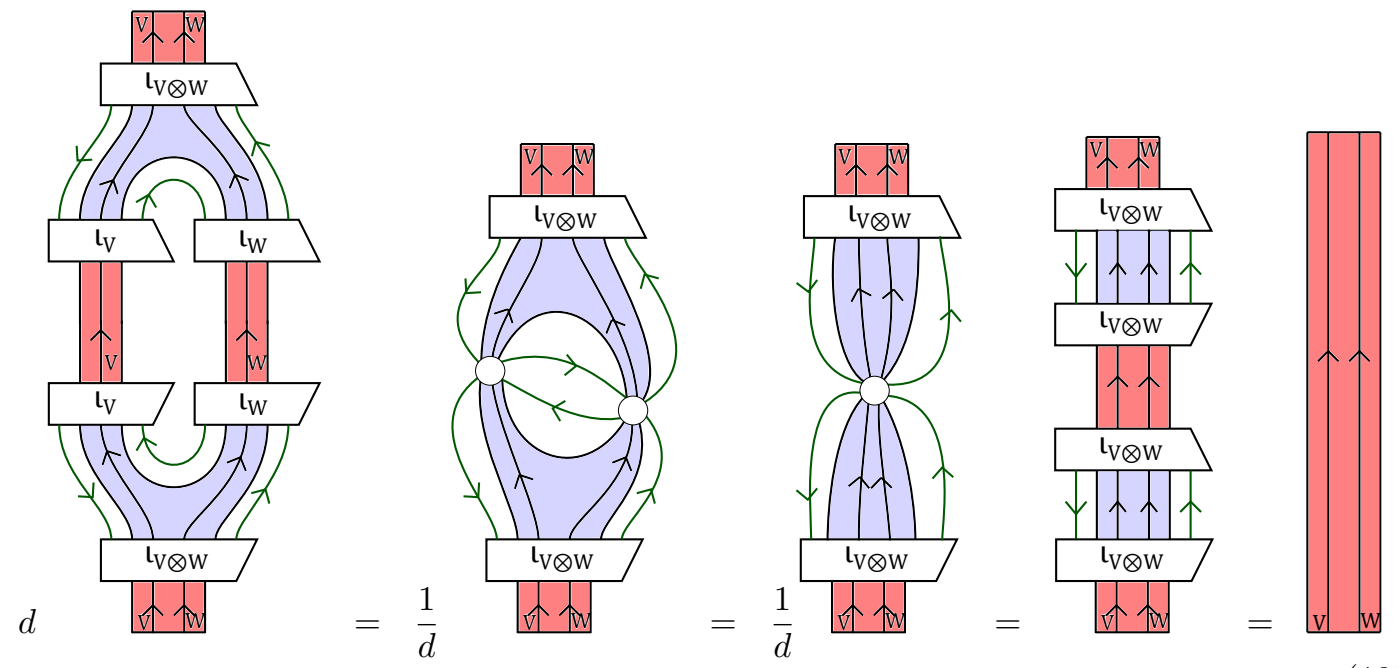

$(108)$

Here for the first equality we used the second equation of (102); for the second equality we used monoidality of $\alpha$; for the third equation we used the second equation of (102); and for the fourth equality we used the first equation of (102). 
For the other unitarity equation:

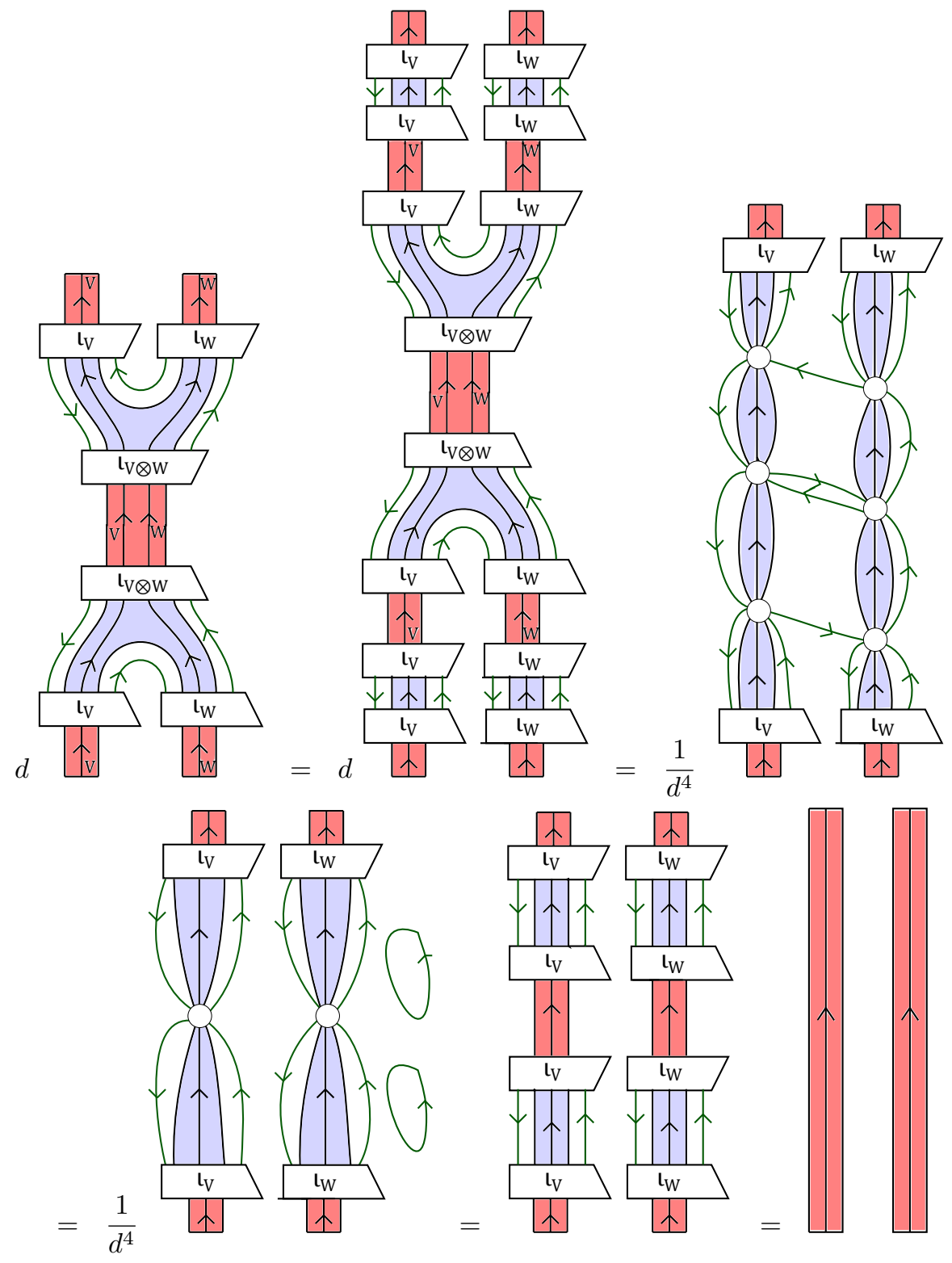

Here for the first equality we used the first equation of (102); for the second equality we used the second equation of (102) and monoidality of $\alpha$; for the third equality we used the fact that the multiplication of the Frobenius algebra is a modification; for the fourth equality we evaluated the loops and used the second equation of (102); for the fifth equality we used the first equation of (102). 
- Unitarity of $u$. For the first equation:

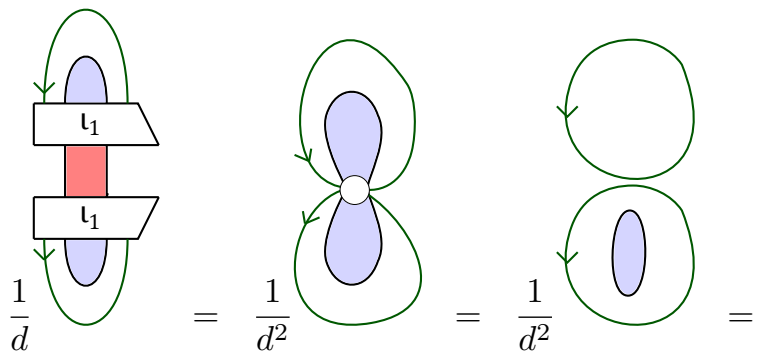

Here for the first equality we used the second equation of (102); for the second equality we used monoidality of $\alpha$ (37); and for the third equality we evaluated the loops and used unitarity of $F$. For the second equation:

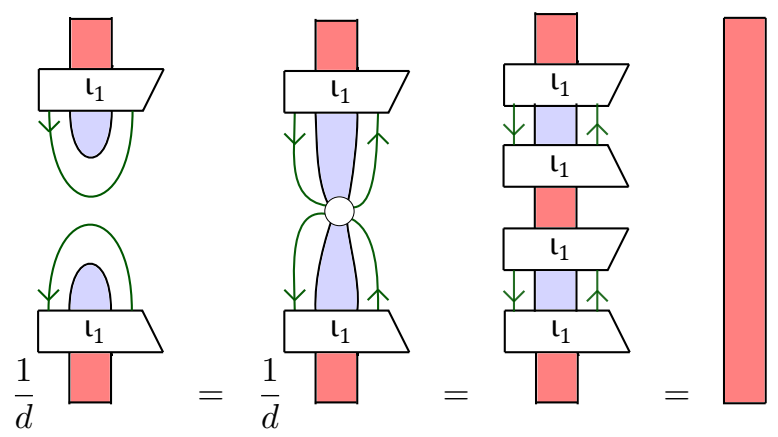

For the first equality we used monoidality of $\alpha$ (37); for the second equality we used the second equation of (102); and for the third equality we used the first equation of (102).

- Associativity (27). We have the following sequence of equations:
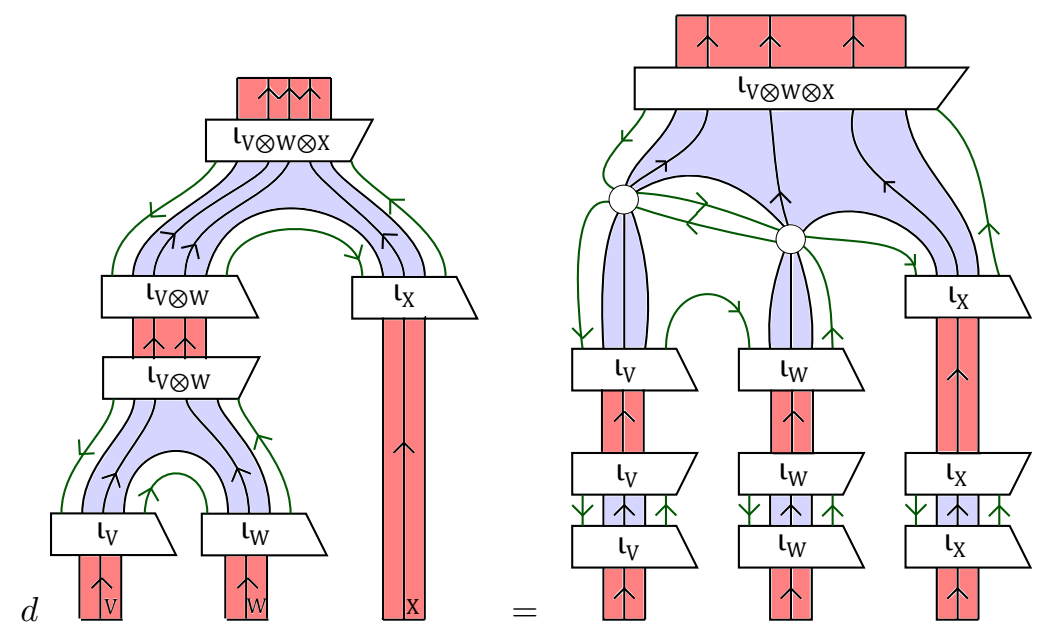


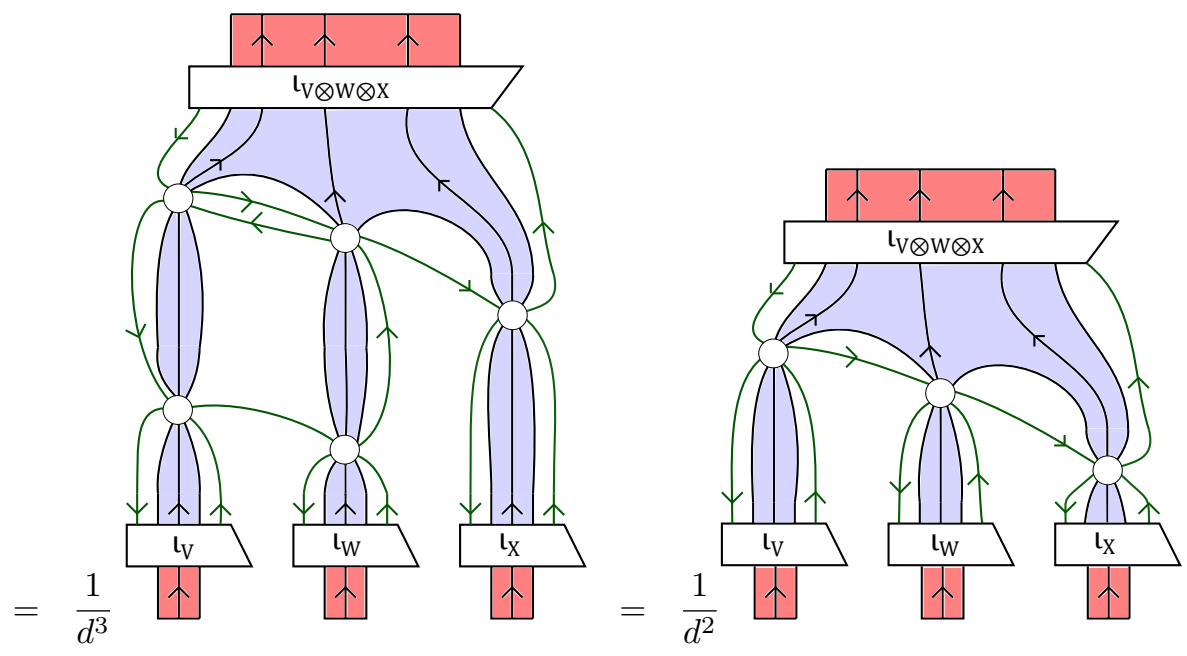

Here for the first equality we used the first equation of (102) to insert isometries and their inverses on all three legs, and the second equation of (102) and monoidality of $\alpha$. For the second equality we used the second equation of (102). For the third equality we used the fact that the multiplication of the Frobenius algebra is a modification and evaluated the resulting loop.

We leave the rest of the proof to the reader: use monoidality of $\alpha$ on the two rightmost legs, use the second equation of (102) to eliminate all occurences of $\alpha$, then cancel isometries using the first equation of (102).

- Unitality (28). The left unitality equation is shown as follows:

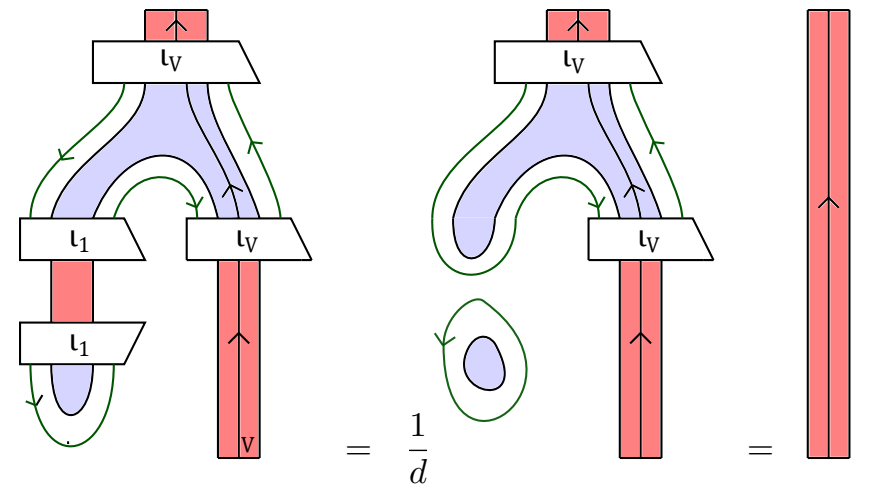

For the first equation we used the second equation of (102) and monoidality of $\alpha$; for the second equation we evaluated the loop and used unitarity of $F_{\alpha}$. The right unitality equation is shown similarly.

We have therefore shown that $F_{\alpha}$ is a fibre functor on $\operatorname{Rep}(G)$. We must now show that $\sqrt{\alpha}$ is a UPT $F \rightarrow F_{\alpha}$. For this we must show naturality and monoidality (35-37). 
- Naturality. For any $f: V \rightarrow W$ :
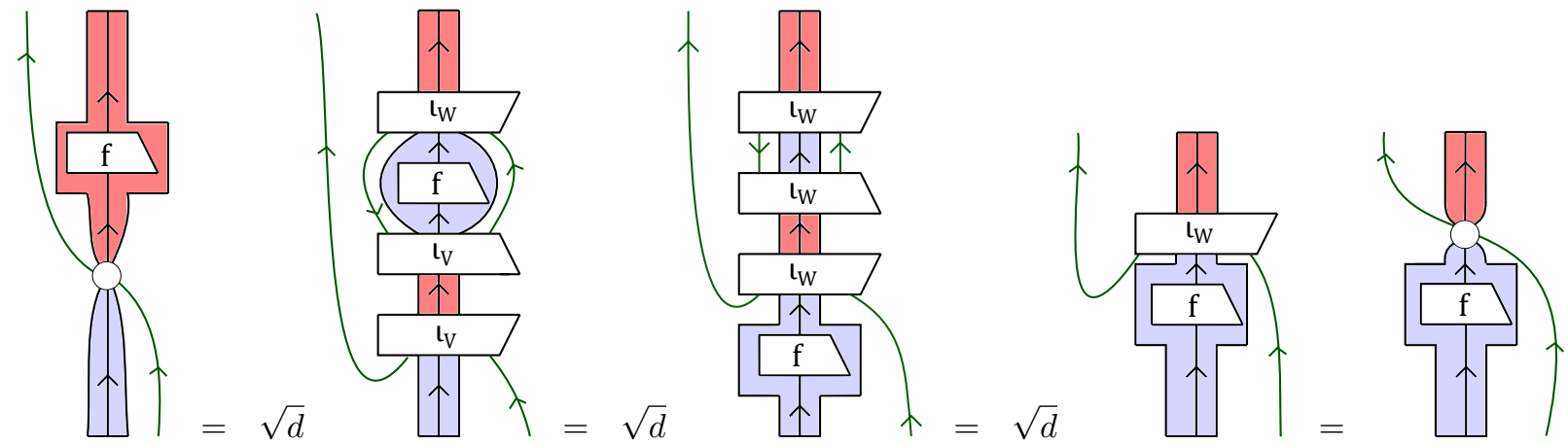

Here the first and fourth equalities are by definition; the second equality is by the second equation of (102) and naturality of $\alpha$; and the third equality is by the first equation of (102).

- Monoidality.

- We show (36):
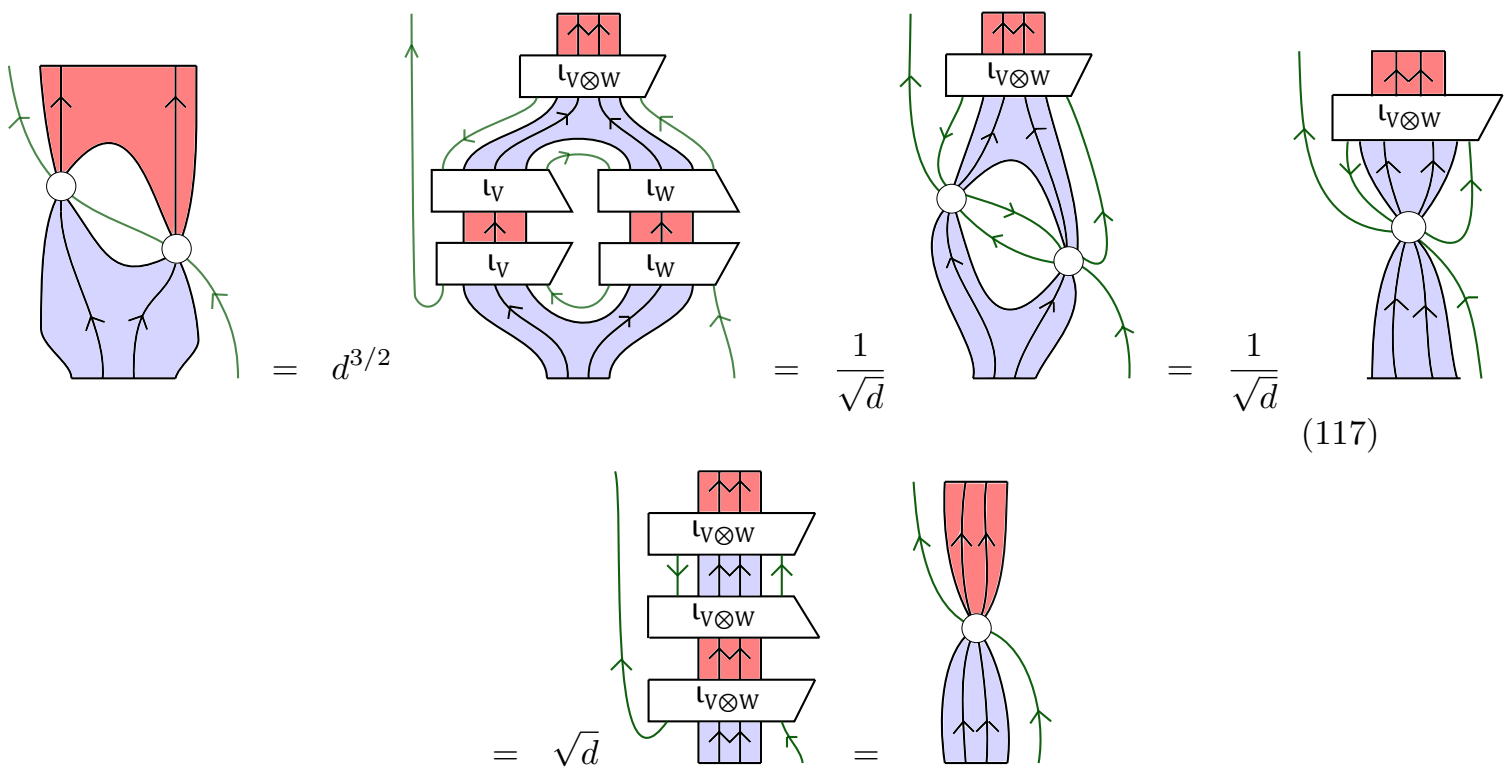

Here the first equality is by definition of $\sqrt{\alpha}$ and the multiplicator of $F_{\alpha}$; the second equality is by the second equation of (102); the third equality is by monoidality of $\alpha$; the fourth equality is by the second equation of (102); and the fifth equality is by the first equation of (102) and the definition of $\sqrt{\alpha}$. 
- We show (37):
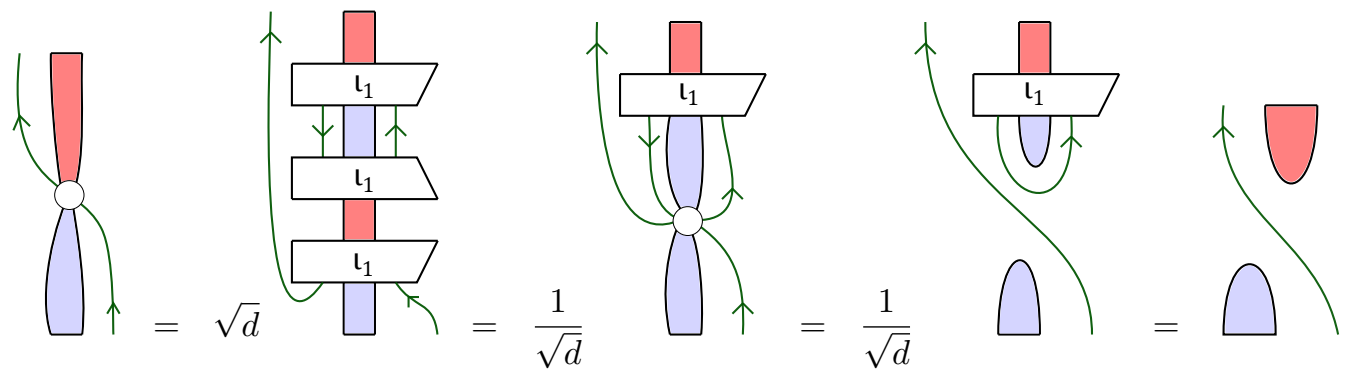

Here the first equality is by definition of $\sqrt{\alpha}$ and by the first equation of (102); the second equality is by the second equation of (102); the third equality is by monoidality of $\alpha$; and the fourth equality is by definition of the unitor of $F_{\alpha}$.

- Unitarity. We show the first equation of unitarity:

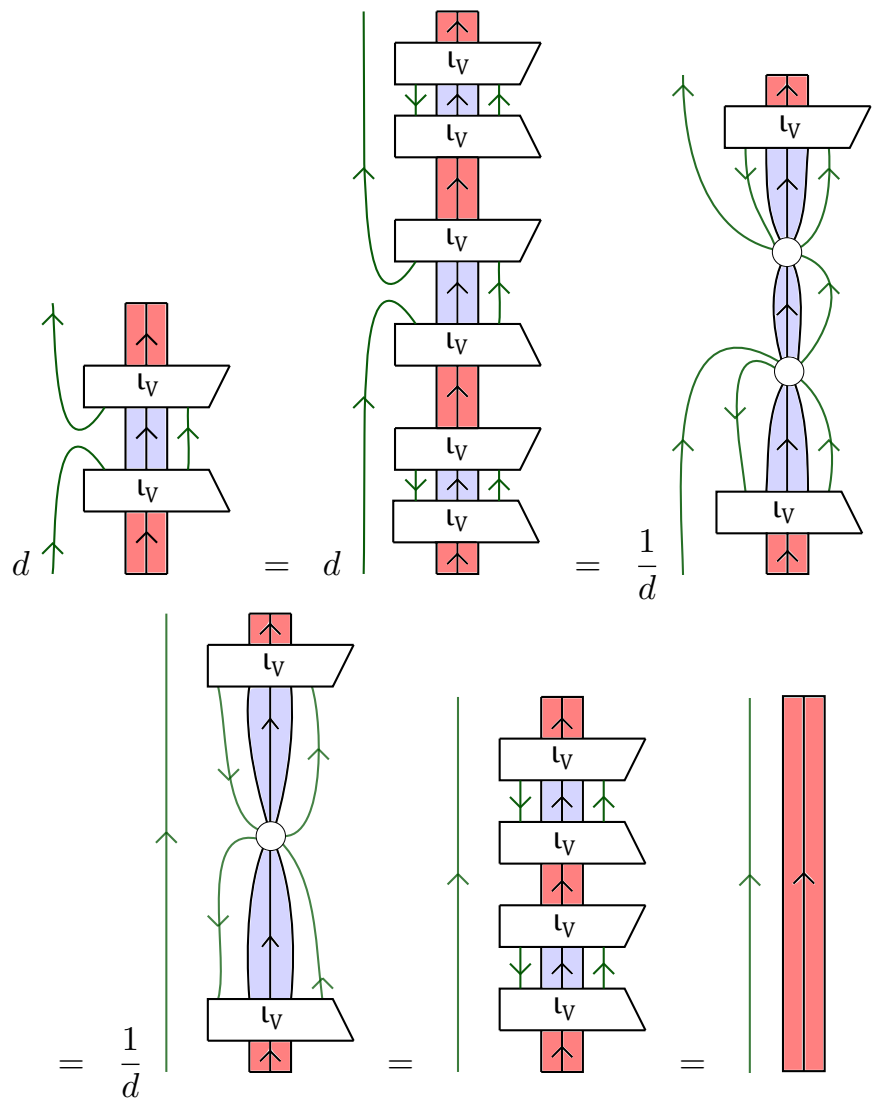

Here for the first equality we used the first equation of (102); for the second equality we used the second equation of (102); for the third equality we used the fact that the comultiplication of $\left(\left(\alpha, H \otimes H^{*}\right), m, u\right)$ is a modification; for the fourth equality we used the second equation of (102); and for the final equality we used the first equation of (102). 
We show the second equation of unitarity:

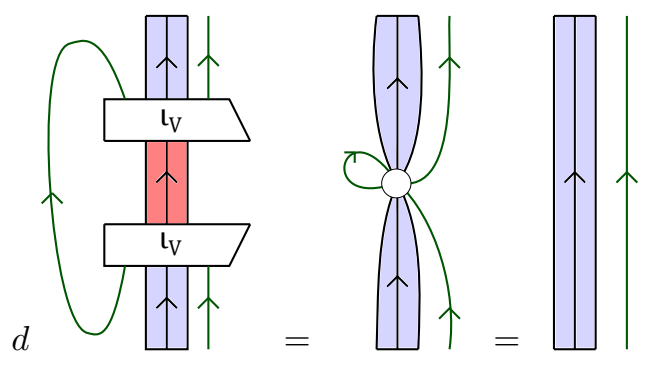

For the first equality we used the second equation of (102), and for the second equality we used that the counit of $\left(\left(\alpha, H \otimes H^{*}\right), m, u\right)$ is a modification.

We have therefore shown that $\alpha: G \rightarrow F$ is a UPT. Finally, we show that $\sqrt{\alpha}$ splits $\alpha$, i.e. $\sqrt{\alpha} \circ \sqrt{\alpha}^{*}=$ $\alpha$. By unitarity of $\sqrt{\alpha}$ it is equivalent to show that $\sqrt{\alpha} \circ \sqrt{\alpha}^{\dagger}=A$, which follows immediately from the second equation of (102):

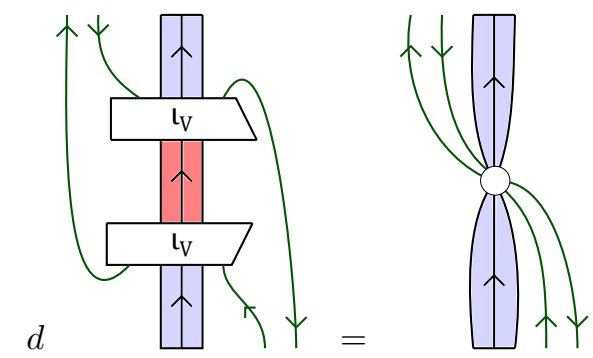

Classification of UPTs and accessible fibre functors. We have seen that every UPT $\alpha: F \rightarrow F^{\prime}$, where $F^{\prime}$ is some fibre functor accessible from $F$, yields a simple Frobenius algebra in $\operatorname{End}(F)$, and that every unitary *-isomorphism class of simple Frobenius algebras is obtained in this way. By means of this correspondence we can classify UPTs and accessible fibre functors.

Definition 4.13. Let $\alpha_{1}: F \rightarrow F_{1}$ and $\alpha_{2}: F \rightarrow F_{2}$ be UPTs. We say that $\alpha_{1}$ and $\alpha_{2}$ are equivalent if there exists a a unitary monoidal natural isomorphism $E: F_{2} \rightarrow F_{1}$ and a unitary modification $\tau: \alpha_{1} \rightarrow \alpha_{2} \otimes E$.

Theorem 4.14. Let $F$ be the canonical fibre functor $\operatorname{Rep}(G) \rightarrow$ Hilb. Then the constructions of Proposition 4.7 and Theorem 4.12 give an explicit bijective correspondence between:

- Unitary monoidal isomorphism classes of unitary fibre functors accessible from F by a UPT; and Morita equivalence classes of simple Frobenius monoids in $\operatorname{Rep}\left(A_{G}\right)$.

- Equivalence classes of UPTs $\alpha: F \rightarrow F^{\prime}$ for some accessible fibre functor $F^{\prime}$; and unitary *-isomorphism classes of simple Frobenius monoids in $\operatorname{Rep}\left(A_{G}\right)$.

Proof. A full proof is given in $[19, \S 6]$ (the relevant theorems are [19, Thm. 6.11, Thm 6.19]). The result is an application of Morita theory in the 2-category $\operatorname{Fun}(\operatorname{Rep}(G), \operatorname{Hilb})$. We have just shown that the special Frobenius monoids in $\operatorname{End}(F)$ which split — i.e. which are isomorphic to 'pair of pants' algebras $\alpha \otimes \alpha^{*}$ for 1-morphisms $\alpha: F \rightarrow F^{\prime}$ - are precisely the simple dagger Frobenius monoids. Morita theory implies a bijective correspondence between Morita equivalence classes of split special Frobenius monoids in $\operatorname{End}(F)$ and equivalence classes of objects $F^{\prime}$ in $\operatorname{Fun}(\operatorname{Rep}(G)$, Hilb) such that there exists a 1-morphism $\alpha: F \rightarrow F^{\prime}$; equivalence of objects in $\operatorname{Fun}(\operatorname{Rep}(G)$, Hilb) corresponds to unitary monoidal 
natural isomorphism of fibre functors, which gives the first bullet point. Finally, Morita theory implies a bijective correspondence between unitary $*$-isomorphism classes of simple Frobenius monoids in $\operatorname{Rep}\left(A_{G}\right)$, and equivalence classes of 1-morphisms $\alpha: F \rightarrow F^{\prime}$ in $\operatorname{Fun}(\operatorname{Rep}(G)$, Hilb). Equivalence of 1-morphisms in this setting comes down to Definition 4.13, whence the second bullet point.

Example 4.15. One application of this result is to construct fibre functors. For example, it is straightforward to use Theorem 4.14 to obtain a concrete construction of all fibre functors on the category $\operatorname{Rep}(G)$ for a finite group $G$, since by Corollary 3.17 they are all accessible by a UPT. It is well-known that they correspond to conjugacy classes of pairs $(H, \psi)$, where $H<G$ is a subgroup of central type and $\psi: H \times H \rightarrow U(1)$ is a nondegenerate 2-cocycle. However, to our knowledge the only known way to construct them was to apply Construction 3.3, which is not quite concrete enough for some applications. Our construction, which uses only very basic representation theory, is stated in [18, $\S 6.1]$.

\section{Quantum graphs and their isomorphisms}

In this Section we give an example of UPTs arising in the study of finite quantum graph theory [14]. Specifically, we will show that finite-dimensional quantum graph isomorphisms from a quantum graph $X$ are UPTs from the canonical fibre functor on the category of representations of its quantum automorphism group.

\subsection{UPTs for compact matrix quantum groups}

Definition 5.1. We say that a $C^{*}$-tensor category $\mathcal{C}$ is generated by a family of objects $Q$ if, for any object $V$ of $\mathcal{C}$, there exists a family $\left\{b_{k}\right\}$ of reduction morphisms $b_{k} \in \operatorname{Hom}\left(r_{k}, V\right)$, where each $r_{k}$ is a monoidal product of objects in $Q$, such that $\sum_{k} b_{k} b_{k}^{\dagger}=\mathrm{id}_{V}$.

Definition 5.2. We say that a pair $(G, u)$ of a compact quantum group $G$ and some representation $u$ is a compact matrix quantum group when $\operatorname{Rep}(G)$ is generated by the objects $\left\{u, u^{*}\right\}$.

For $(G, u)$ a compact matrix quantum group, we will now show that a UPT between fibre functors on $\operatorname{Rep}(G)$ is completely determined by its component on the fundamental representation $u$.

First we define some notation: for a vector $\vec{x} \in\{ \pm 1\}^{n}, n \in \mathbb{N}$, we write $u^{\vec{x}}$ for the object $u^{x_{1}} \otimes$ $\cdots \otimes u^{x_{n}}$, where we take $u^{-1}:=u^{*}$. We additionally define $u^{0}:=\mathbb{1}$.

Definition 5.3. Let $(G, u)$ be a compact matrix quantum group, and let $F, F^{\prime}: \operatorname{Rep}(G) \rightarrow$ Hilb be fibre functors. We define a reduced unitary pseudonatural transformation $(\tilde{\alpha}, H): F \rightarrow F^{\prime}$ to be:

- A Hilbert space $H$ (drawn as a green wire).

- A unitary morphism $\tilde{\alpha}: F(u) \otimes H \rightarrow H \otimes F^{\prime}(u)$ (drawn as a white vertex) which is:

- Natural. For any 2-morphism $f: u^{\vec{x}} \rightarrow u^{\vec{y}}$ in $\mathcal{C}$ :

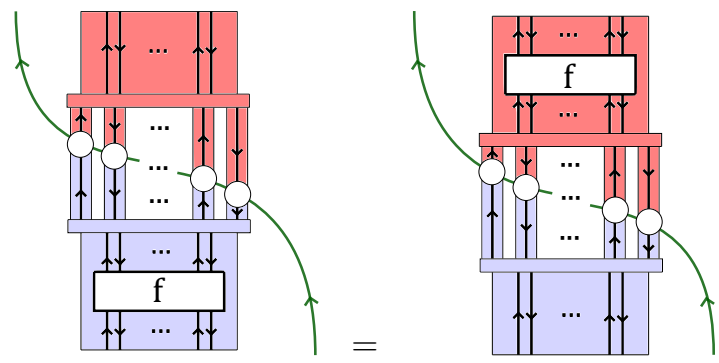


Here the empty horizontal blue and red rectangles represent some sequence of multiplicators, comultiplicators, unitors and counitors. For the purpose of drawing the diagram (124) we have supposed that $\vec{x}, \vec{y}$ are both of the form $(1,-1, \ldots, 1,-1)$; it should be clear how to generalise to other $\vec{x}, \vec{y}$ or to $u^{0}$ (e.g. if $f: u^{0} \rightarrow u^{\vec{y}}$, on the RHS of (124) the blue rectangle will be the counitor of $F$, the red will be the unitor of $F^{\prime}$ and there will be no white vertices). We also used the following definition:

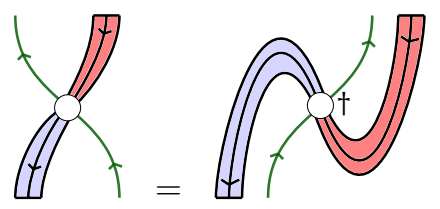

It is immediate from Definition 2.30 and unitarity of $\alpha(40)$ that a UPT $\alpha: F \rightarrow F^{\prime}$ restricts to a reduced UPT $\alpha_{u}$. We now show that this correspondence is bijective: every reduced UPT induces a unique UPT.

Lemma 5.4. If $\tilde{\alpha}$ is a reduced UPT, then the morphism defined in (125) is unitary.

Proof. We show one of the two unitarity equations; the other is proved similarly.

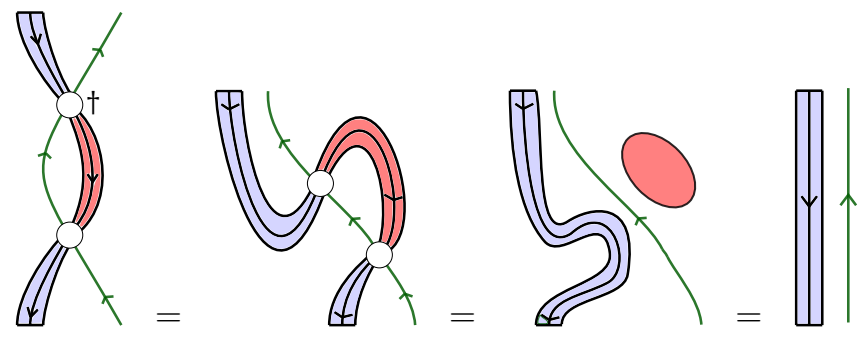

Here the first equality is by definition (125), the second is by naturality (124) of the reduced UPT $\tilde{\alpha}$ for the morphism $\epsilon: u^{(-1,1)} \rightarrow u^{0}$, and the third is by unitarity of the monoidal functor $F^{\prime}$.

Proposition 5.5. Let $(G, u)$ be a compact matrix quantum group, let $F, F^{\prime}$ be fibre functors, and let $(\tilde{\alpha}, H): F \rightarrow F^{\prime}$ be a reduced UPT. There is a unique UPT $(\alpha, H): F \rightarrow F^{\prime}$ which restricts to $\tilde{\alpha}$ on $\alpha_{u}$, whose components $\alpha_{V}$ are defined as follows for any representation $V$ of $G$ :

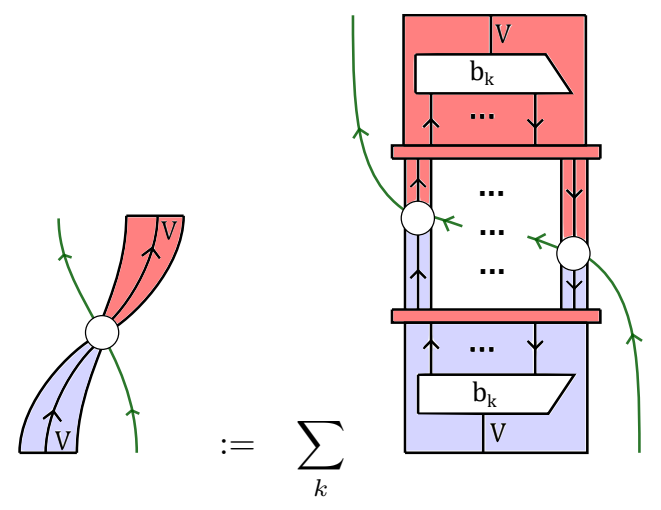

Here $\left\{b_{k}: u^{\overrightarrow{x_{k}}} \rightarrow V\right\}$ is any family of reduction morphisms.

Proof. First we show that $\alpha$ is well-defined, i.e. it does not depend on the choice of reduction morphisms. Let $V$ be some representation of $G$ and let $\left\{b_{k}: u^{\overrightarrow{x_{k}}} \rightarrow V\right\}$ and $\left\{c_{l}: u^{\overrightarrow{y_{l}}} \rightarrow V\right\}$ be two families 
of reduction morphisms. Then:

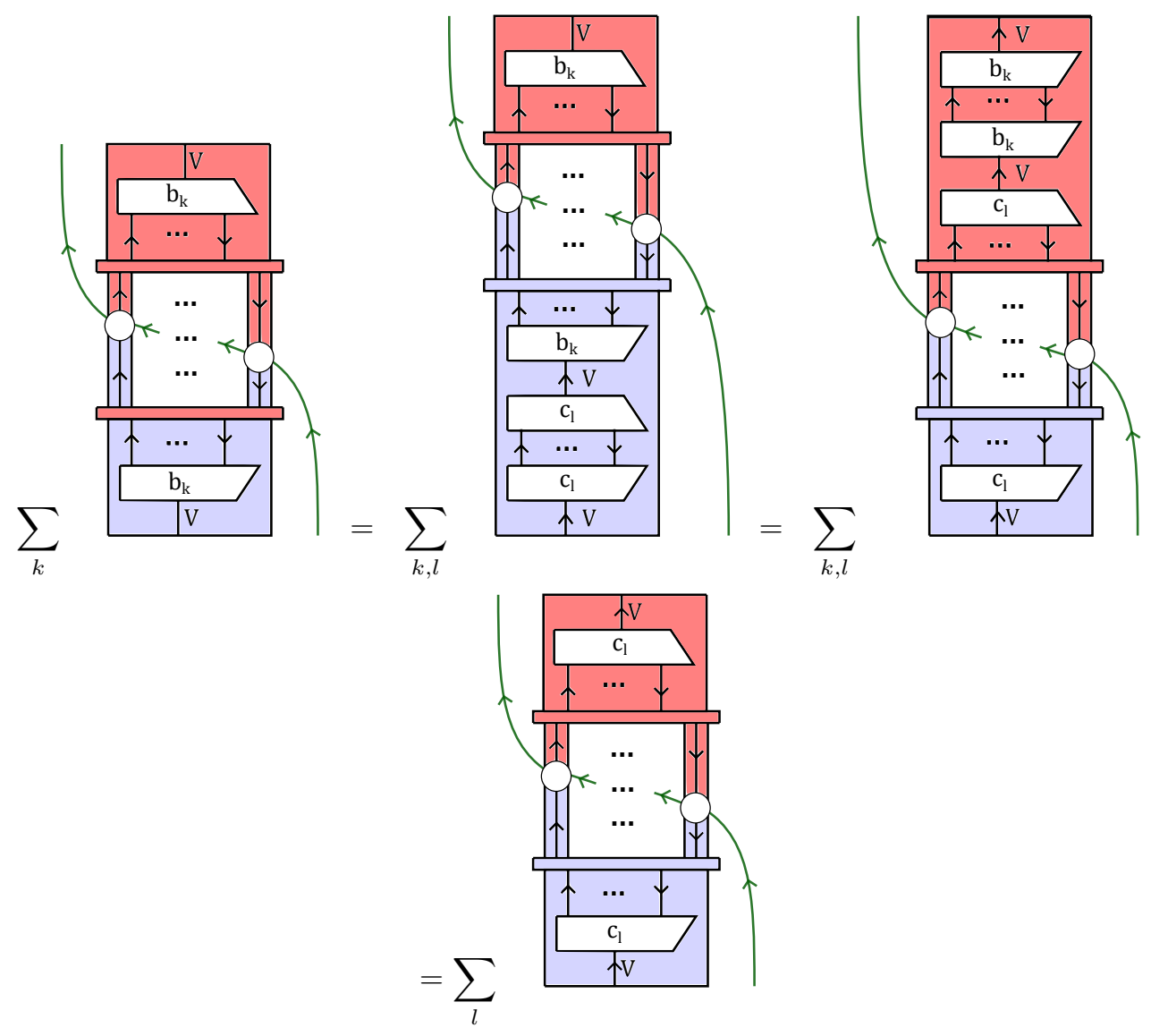

Here the first equality is by $\sum_{l} c_{l} c_{l}^{\dagger}=\mathrm{id}_{V}$, the second is by naturality of the reduced UPT $\tilde{\alpha}$, and the third is by $\sum_{k} b_{k} b_{k}^{\dagger}=\mathrm{id}_{V}$.

We now show that $\alpha$ is indeed a UPT.

- Naturality. Let $\left\{b_{k}: u^{\vec{x}_{k}} \rightarrow V\right\}$ and $\left\{c_{l}: u^{\vec{y}_{l}} \rightarrow W\right\}$ be reduction morphisms for representations $V, W$ of $\mathcal{C}$. We show (35) for any morphism $f: V \rightarrow W$ :

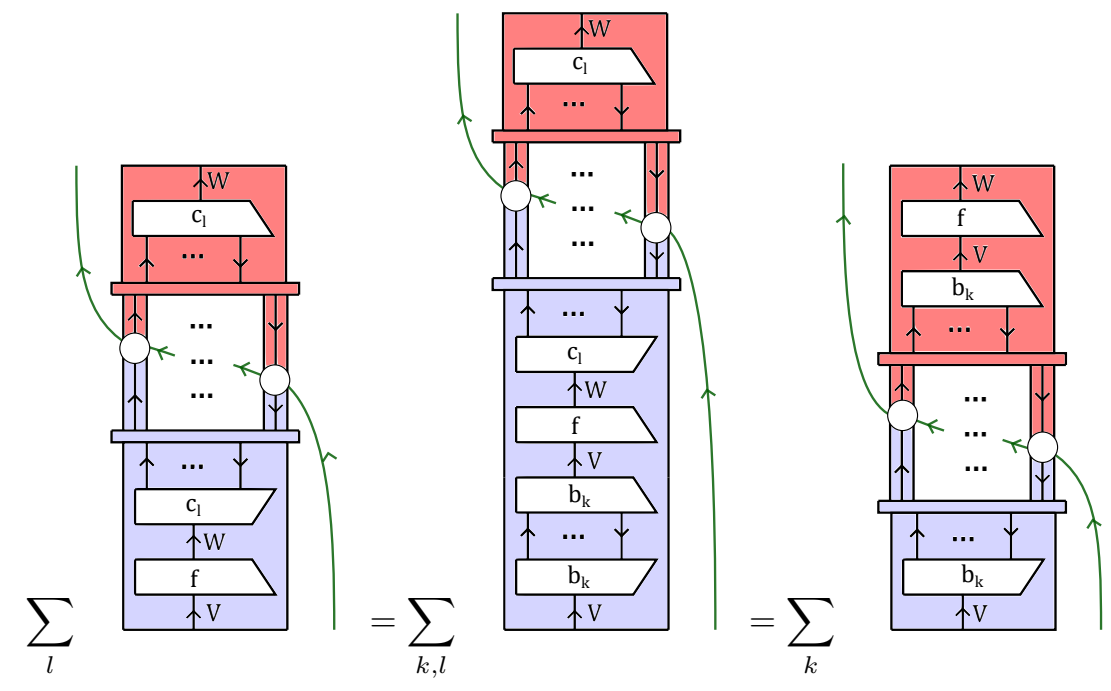


Here the first equality is by $\sum_{k} b_{k} b_{k}^{\dagger}=\mathrm{id}_{V}$, and the second is by naturality of the reduced UPT $\tilde{\alpha}$ and $\sum_{l} c_{l} c_{l}^{\dagger}=\mathrm{id}_{W}$.

\section{- Monoidality.}

- Let $V, W$ be some representations of $G$, and pick some reduction morphisms $\left\{b_{k}: u^{\vec{x}_{k}} \rightarrow\right.$ $V\},\left\{c_{l}: u^{\vec{y}_{l}} \rightarrow W\right\}$. It is clear that $\left\{b_{k} \otimes c_{l}: u^{\vec{x}_{k}} \otimes u^{\vec{y}_{l}} \rightarrow V \otimes W\right\}$ are reduction morphisms for $V \otimes W$. Now (36) is immediate by manipulation of functorial boxes:

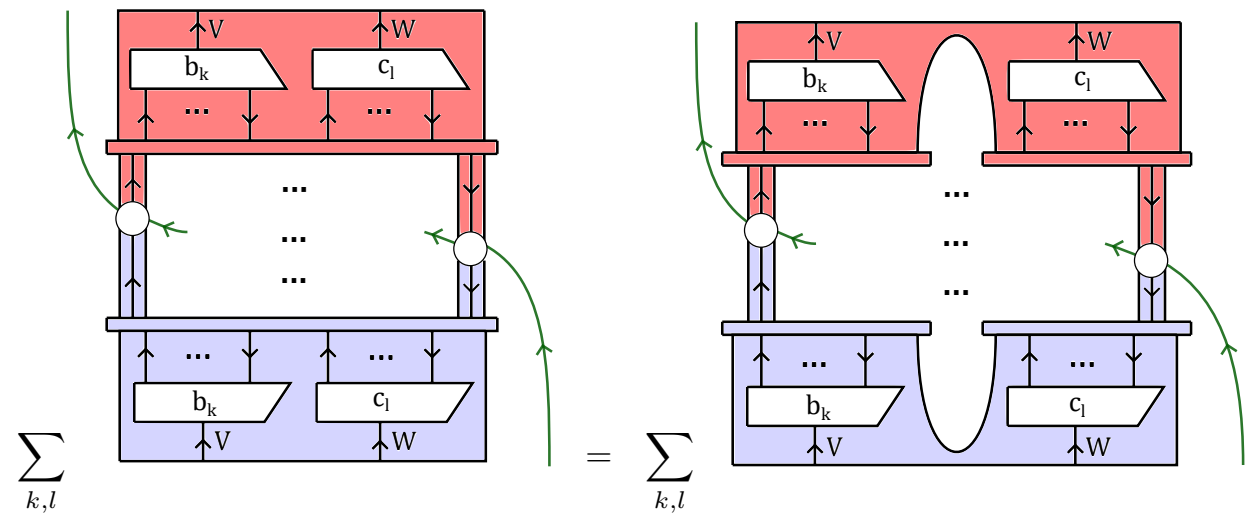

- The equation (37) is precisely (127) for the object $\mathbb{1}$ where the reduction morphism is $\operatorname{id}_{\mathbb{1}}: \mathbb{1} \rightarrow u^{0}$.

- Unitarity. We show one of the unitarity equations; the other is proved similarly. For any representation $V$ of $G$ :

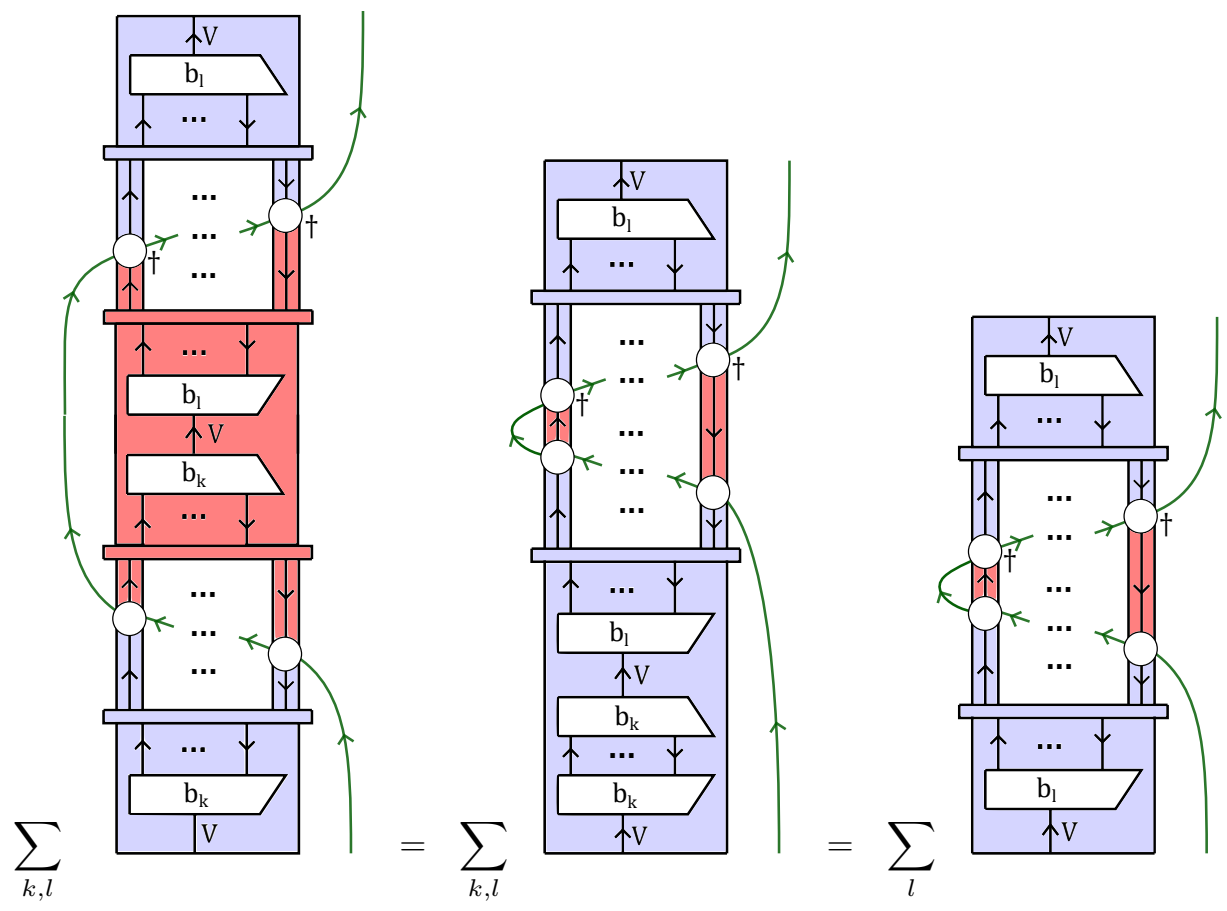




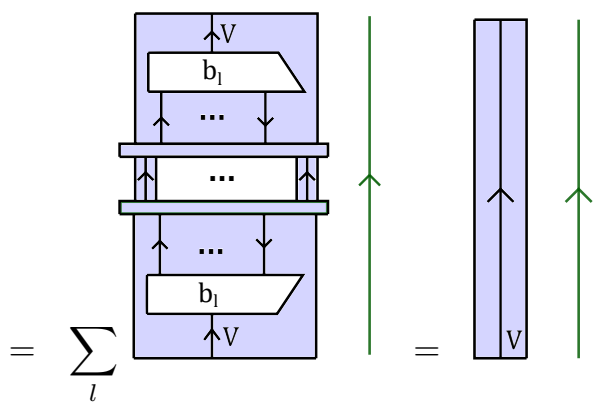

Here the first equation is by naturality for $\tilde{\alpha}$, the second is by $\sum_{k} b_{k} b_{k}^{\dagger}=\mathrm{id}_{V}$, the third is by unitarity of $\alpha_{u}$ and Lemma 5.4, and the fourth is by manipulation of functorial boxes and $\sum_{l} b_{l} b_{l}^{\dagger}=\mathrm{id} V$.

Finally, uniqueness of the induced UPT $\alpha$ follows from the fact that, by naturality of the UPT $\alpha: F \rightarrow F^{\prime}$, the component $\alpha_{V}$ for any $V$ is defined by $\alpha_{U}$ and $\alpha_{U^{*}}$ by (127).

We can also introduce a notion of modification for reduced UPTs.

Definition 5.6. Let $(G, u)$ be a compact matrix quantum group, let $F, F^{\prime}$ be fibre functors, and let $\left(\tilde{\alpha}, H_{\alpha}\right),\left(\tilde{\beta}, H_{\beta}\right)$ be reduced UPTs (the first drawn with a green wire, the second with an orange wire). Then a modification $f: \tilde{\alpha} \rightarrow \tilde{\beta}$ is a linear map $f: H_{\alpha} \rightarrow H_{\beta}$ satisfying the following equations:
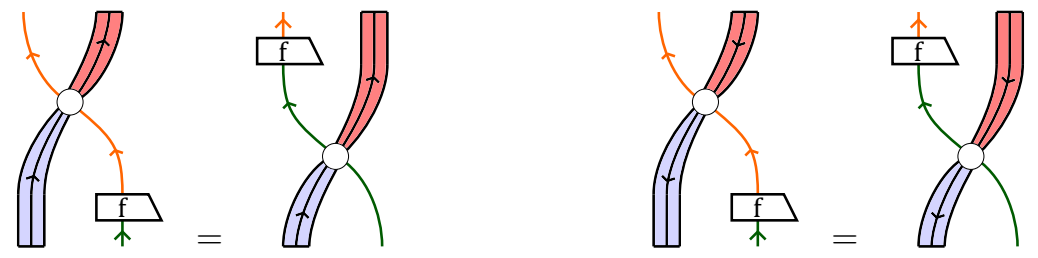

Proposition 5.7. Let $\left(\tilde{\alpha}, H_{\alpha}\right),\left(\tilde{\beta}, H_{\beta}\right): F \rightarrow F^{\prime}$ be reduced UPTs and let $\left(\alpha, H_{\alpha}\right),\left(\beta, H_{\beta}\right): F \rightarrow F^{\prime}$ be the unique induced UPTs. Then a modification $f: \tilde{\alpha} \rightarrow \tilde{\beta}$ is precisely a modification $f: \alpha \rightarrow \beta$.

Proof. It is clear that every modification $\alpha \rightarrow \beta$ is in particular a modification $\tilde{\alpha} \rightarrow \tilde{\beta}$. That a modification $f: \tilde{\alpha} \rightarrow \tilde{\beta}$ is also a modification $f: \alpha \rightarrow \beta$ is clear from the definition (127) of the induced UPTs; $f$ pulls through all the vertices, thereby satisfying the equations (39).

The results of this section are summarised by the following theorem.

Theorem 5.8. Let $(G, u)$ be a compact matrix quantum group and let $F, F^{\prime}$ be fibre functors. Then restriction of UPTs $\alpha \mapsto \alpha_{u}$ defines an isomorphism of categories between:

- UPTs $F \rightarrow F^{\prime}$ and modifications.

- Reduced UPTs $F \rightarrow F^{\prime}$ and modifications.

\subsection{Quantum graphs and their isomorphisms}

We have seen that for a compact matrix quantum group $(G, u)$, a UPT $\alpha: F_{1} \rightarrow F_{2}$ between fibre functors $F_{1}, F_{2}: \operatorname{Rep}(G) \rightarrow$ Hilb is determined by a single unitary $\left(\alpha_{U}, H\right): F_{1}(u) \otimes H \rightarrow H \otimes F_{2}(u)$ obeying the naturality condition (124) for the intertwiner spaces $\operatorname{Hom}\left(u^{\otimes m}, u^{\otimes n}\right)$.

We now recall the notions of quantum confusability graph and finite-dimensional quantum graph isomorphism. For more information about these structures, their significance in quantum information theory, and how they generalise their classical counterparts, see $[1,5,6,14]$.

Definition 5.9 ([14, Def. 5.1]). A quantum graph $X=(A, \Gamma)$ is a pair of: 
- A special Frobenius monoid $(A, m, u)$ in the category Hilb (Definition 4.1) satisfying the following symmetry equation:

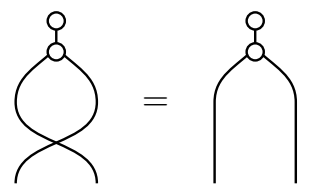

- A self-adjoint linear map $\Gamma: A \rightarrow A$ satisfying the following equations:
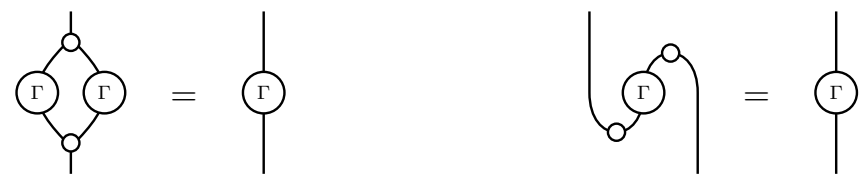

Example 5.10. We briefly recall how quantum graphs generalise ordinary graphs. Let $\{|i\rangle\}$ be an orthonormal basis for a $d$-dimensional Hilbert space $A$. This Hilbert space has the structure of a special symmetric Frobenius algebra in Hilb with the following operations:

$$
\begin{array}{ccrl}
|i\rangle \otimes|j\rangle \mapsto \delta_{i j}|i\rangle & 1 \mapsto \sum_{i}|i\rangle & |i\rangle \mapsto|i\rangle \otimes|i\rangle & |i\rangle \mapsto 1 \\
\text { multiplication } & \text { unit } & \text { comultiplication } & \text { counit }
\end{array}
$$

We can express any linear map $\Gamma: A \rightarrow A$ in the basis $\{|i\rangle\}$ as a matrix $\left(\Gamma_{i j}\right)_{i, j}$. It is then easy to check that (136) reduces to the following conditions for the matrix entries:

$$
\Gamma_{i j}^{2}=\Gamma_{i j} \quad \Gamma_{i j}=\Gamma_{j i}
$$

These are clearly the equations specifying that $\Gamma$ is the adjacency matrix for a graph with $d$ vertices, i.e. a symmetric $d \times d$ matrix with entries 0 and 1 . In order to restrict to e.g. simple graphs we could add another equation, but we do not do this here.

Definition 5.11 ([14, Def. 5.11]). Let $X=(A, \Gamma)$ and $Y=\left(A^{\prime}, \Gamma^{\prime}\right)$ be quantum graphs. A finitedimensional quantum graph isomorphism $(P, H): X \rightarrow Y$ is a pair of a Hilbert space $H$ and a unitary linear map $P: A \otimes H \rightarrow H \otimes A^{\prime}$ satisfying the following equations, where the monoids $A$ and $A^{\prime}$ are depicted as white and grey nodes respectively:
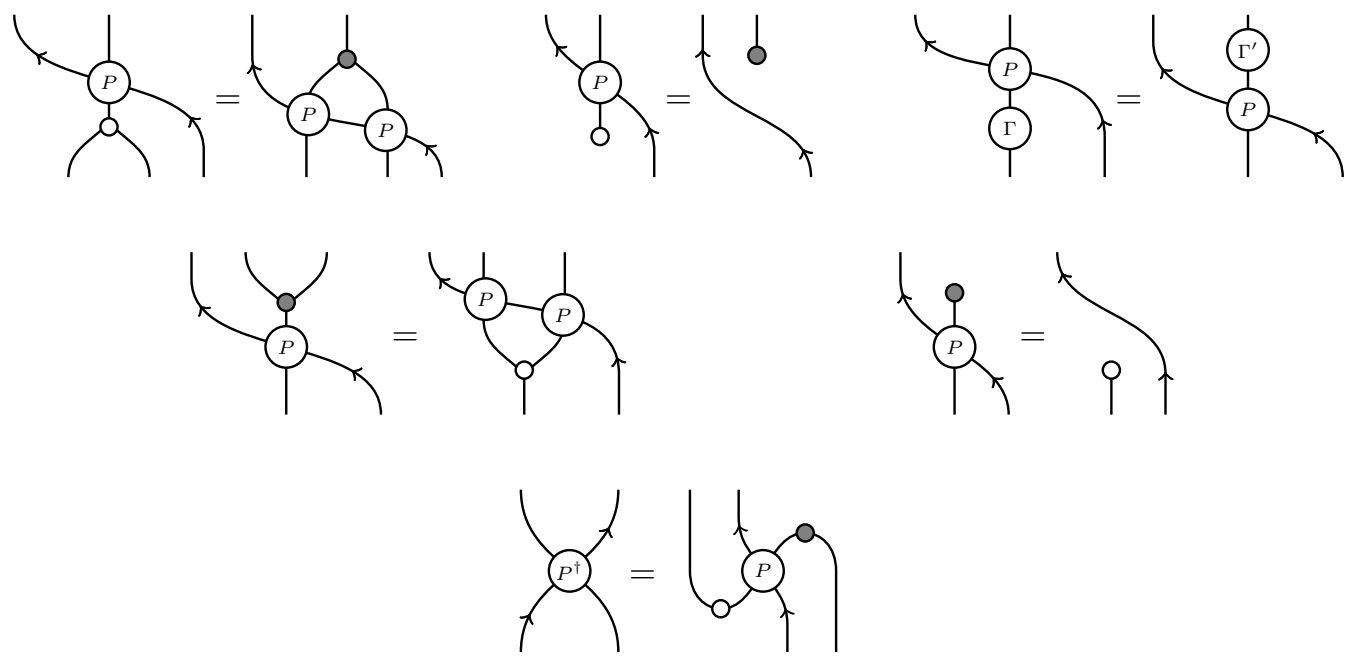
An intertwiner of quantum isomorphisms $(H, P) \rightarrow\left(H^{\prime}, P^{\prime}\right)$ is a linear map $f: H \rightarrow H^{\prime}$ such that the following holds:

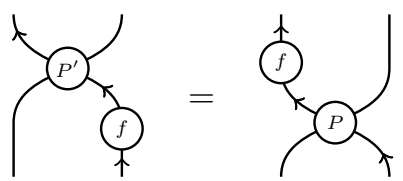

Remark 5.12. This definition uses the opposite convention for the direction of the Hilbert space wire to the paper [14]. The theory of quantum isomorphisms can be developed analogously whichever convention is used.

We now recall the definition of the quantum automorphism group of a quantum graph.

Definition 5.13 ([5, Def. 3.7]). Let $X=(A, \Gamma)$ be a quantum graph with $\operatorname{dim}(A)=n$, and let $\{|i\rangle\}_{i=1}^{n}$ be an orthonormal basis for $A$. Then the quantum automorphism group algebra $O\left(G_{X}\right)$ is the universal unital $*$-algebra generated by the coefficients of a unitary matrix $\left[u_{i j}\right]_{i, j=1}^{n} \in M_{n}\left(O\left(G_{X}\right)\right)$ subject to the relations making the map

$$
\rho: A \rightarrow A \otimes O\left(G_{X}\right) \quad \rho(|i\rangle)=\sum_{j}|j\rangle \otimes u_{j i}
$$

a unital $*$-homomorphism satisfying $\rho \circ \Gamma=\left(\Gamma \otimes \operatorname{id}_{O\left(G_{X}\right)}\right) \circ \rho$. Its Hopf- $*$-algebra structure is defined in [5, Prop. 3.8].

Remark 5.14. $O\left(G_{X}\right)$ can also be defined as the *-algebra of matrix coefficients of corepresentations of the $C^{*}$-algebra obtained by Woronowicz's Tannaka-Krein construction [20] for a suitable concrete $W^{*}$-category (c.f. [2, Prop. 1.1]). In particular, we have the following facts:

1. $G_{X}$ is a compact matrix quantum group with fundamental representation $A$.

2. The intertwiner spaces $\operatorname{Hom}_{\operatorname{Rep}\left(G_{X}\right)}\left(A^{\otimes m}, A^{\otimes n}\right)$ are generated by three morphisms $m: A \otimes A \rightarrow$ $A, u: \mathbb{C} \rightarrow A$ and $\Gamma: A \rightarrow A$, satisfying the equations of a Frobenius monoid and of a quantum graph, under composition, monoidal product, dagger and linear combination.

3. The fundamental representation $A$ is self-dual in $\operatorname{Rep}\left(G_{X}\right)$ with cup and cap (82).

4. The image of $((A, m, u), \Gamma)$ under the canonical fibre functor $F: \operatorname{Rep}\left(G_{X}\right) \rightarrow$ Hilb is the quantum graph $X$.

Definition 5.15 ([5, Def. 4.1]). Let $X=\left(A_{X}, \Gamma_{X}\right), Y=\left(A_{Y}, \Gamma_{Y}\right)$, be two quantum graphs, where $\operatorname{dim}\left(A_{X}\right)=n, \operatorname{dim}\left(A_{Y}\right)=m$, and let $\{|i\rangle\}_{i=1}^{n}$ and $\{|j\rangle\}_{j=1}^{m}$ be orthonormal bases for $A_{X}$ and $A_{Y}$ respectively. Define $O\left(G_{Y}, G_{X}\right)$ to be the universal *-algebra generated by the coefficients of a unitary matrix $p=\left[p_{i j}\right]_{i j} \in O\left(G_{Y}, G_{X}\right) \otimes B\left(A_{X}, A_{Y}\right)$ with relations ensuring that

$$
\rho_{Y, X}: A_{X} \rightarrow A_{Y} \otimes O\left(G_{Y}, G_{X}\right) \quad \rho_{Y, X}(|i\rangle)=\sum_{j}|j\rangle \otimes p_{j i}
$$

is a unital $*$-homomorphism satisfying $\rho \circ \Gamma_{X}=\left(\Gamma_{Y} \otimes \operatorname{id}_{O\left(G_{Y}, G_{X}\right)}\right) \circ \rho$.

Lemma 5.16. Let $G_{X}$ be the automorphism group of a quantum graph $F(X)=((F(A), F(m), F(u)), F(\Gamma))$, where $F: \operatorname{Rep}\left(G_{X}\right) \rightarrow$ Hilb is the canonical fibre functor, $A$ is the generating object of $\operatorname{Rep}\left(G_{X}\right)$ and $m, u, \Gamma$ are the generating morphisms. Let $F^{\prime}: \operatorname{Rep}(G) \rightarrow$ Hilb be any other fibre functor accessible 
from $F$ by a UPT. Then $F^{\prime}(X):=\left(\left(F^{\prime}(A), F^{\prime}(m), F^{\prime}(u)\right), F^{\prime}(\Gamma)\right)$ is a quantum graph $f . d$. quantum isomorphic to $X$ :
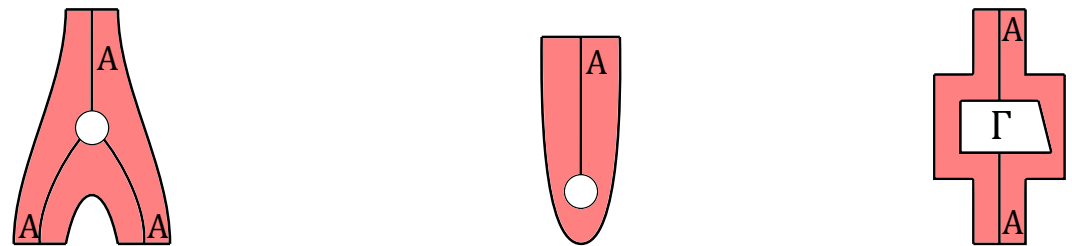

$F^{\prime}(m): F^{\prime}(A) \otimes F^{\prime}(A) \rightarrow F^{\prime}(A)$

$F^{\prime}(u): \mathbb{C} \rightarrow F^{\prime}(A)$

$F^{\prime}(\Gamma): F^{\prime}(A) \rightarrow F^{\prime}(A)$

All quantum graphs f.d. quantum isomorphic to $F(X)$ are obtained in this way.

Proof. All axioms of a quantum graph except symmetry follow straightforwardly from unitarity of the functor $F^{\prime}$ (we need Lemma 2.18 for the Frobenius axiom). For the symmetry condition (135), we recall that any accessible fibre functor has the form given in Theorem (4.12). We can then show symmetry of $F^{\prime}(X)$ as follows:
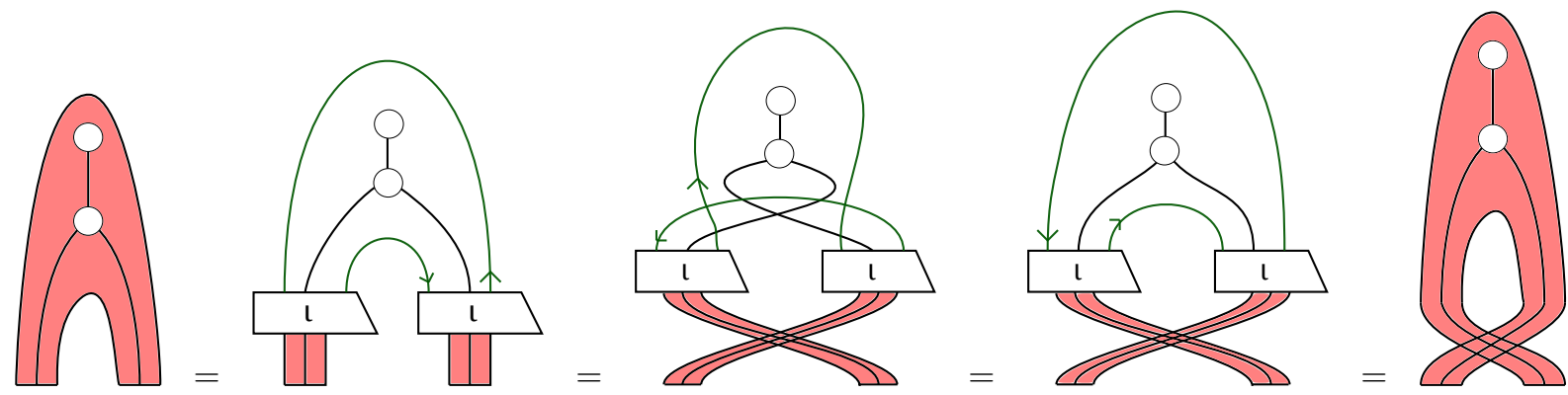

(145)

Here for the first equality we used the definition of the accessible fibre functor $F^{\prime}$ (Theorem 4.12); for the second equality pulled one leg over the other and undid twists in the green wires; for the third equality we used symmetry of $F(X)$; and for the fourth equality we again used the definition of $F^{\prime}$.

It follows that $F^{\prime}(X)$ is a quantum graph. To see that $F^{\prime}(X)$ is f.d. quantum isomorphic to $F(X)$, consider any UPT $\alpha: F \rightarrow F^{\prime}$. Then its restriction $\alpha_{A}$ is a quantum isomorphism: the equations (140) follow from monoidality and naturality of $\alpha$, and the equation (141) follows from unitarity of $\alpha$ (40) and self-duality of $A$.

To see that all quantum graphs f.d. quantum isomorphic to $F(X)$ are thus obtained, we observe that a f.d. quantum isomorphism $P: Y \rightarrow X$ is an f.d. *-representation of $O\left(G_{X}, G_{Y}\right)$, which is therefore nonzero. By [5, Thm. 4.5] $O\left(G_{X}, G_{Y}\right)$ is an $O\left(G_{X}\right)$-Hopf-Galois object and the associated fibre functor $\operatorname{Rep}\left(G_{X}\right) \rightarrow$ Hilb takes $(A, m, u, \Gamma) \mapsto Y$. This fibre functor $F^{\prime}$ is accessible from $F$ precisely because $O\left(G_{X}, G_{Y}\right)$ possesses a finite dimensional *-representation (Theorem 3.14).

Lemma 5.17. Let $X=(A, \Gamma)$ be a quantum graph, let $G_{X}$ be its quantum automorphism group and let $F_{1}, F_{2}: \operatorname{Rep}\left(G_{X}\right) \rightarrow$ Hilb be two fibre functors accessible from the canonical fibre functor. Then there is an isomorphism of categories between:

- UPTs $F_{1} \rightarrow F_{2}$, and modifications.

- Finite-dimensional quantum isomorphisms $F_{1}(X) \rightarrow F_{2}(X)$, and intertwiners.

Proof. We observe that a finite-dimensional quantum isomorphism $F_{1}(X) \rightarrow F_{2}(X)$ is precisely a reduced UPT $F_{1} \rightarrow F_{2}$. Indeed, $A$ is the generating object for $\operatorname{Rep}\left(G_{X}\right)$ and the quantum isomorphism is a unitary linear map of the right type. We must therefore show the naturality equations (124). But 
these are given on generators precisely by (140), which is sufficient. The equation (141) follows from self-duality of $A$.

We also observe that an intertwiner of finite-dimensional quantum graph isomorphisms is precisely a modification of reduced UPTs. This follows from self-duality of $A$, which reduces the equations (134) to (142).

The result then follows immediately from Theorem 5.8.

We now state the main result of this section.

Definition 5.18. Let $X$ be a quantum graph. The 2-category $\mathrm{QGraph}_{X}$ is defined as follows:

- Objects: Quantum graphs f.d. quantum isomorphic to $X$.

- 1-morphisms: Finite-dimensional quantum isomorphisms.

- 2-morphisms: Intertwiners.

Definition 5.19. Let $X$ be a quantum graph. The 2-category $\operatorname{Fun}\left(\operatorname{Rep}\left(G_{X}, \operatorname{Hilb}\right)\right)_{F}$ is defined as follows:

- Objects: Fibre functors accessible from the canonical fibre functor $F: \operatorname{Rep}\left(G_{X}\right) \rightarrow$ Hilb.

- 1-morphisms: UPTs.

-2-morphisms: Modifications.

Theorem 5.20. Let $X$ be a quantum graph. Then there is a 2-equivalence $\operatorname{Fun}\left(\operatorname{Rep}\left(G_{X}\right), \operatorname{Hilb}\right)_{F} \simeq$ QGraph $_{X}$. Moreover, this 2-equivalence is an isomorphism on Hom-categories.

Proof. We define a strict 2-functor $\operatorname{Fun}\left(\operatorname{Rep}\left(G_{X}, \operatorname{Hilb}\right)_{F} \rightarrow \mathrm{QGraph}_{X}\right.$ witnessing the equivalence as follows.

- On objects: An accessible fibre functor $F^{\prime}: \operatorname{Rep}(G) \rightarrow$ Hilb is taken to the quantum graph (143).

- On 1-morphisms: A UPT $\alpha: F^{\prime} \rightarrow F^{\prime \prime}$ is taken to its component $\alpha_{A}$.

- On 2-morphisms: A modification $\alpha \rightarrow \beta$ is taken to an intertwiner $\alpha_{A} \rightarrow \beta_{A}$.

We first show that this is a well-defined strict 2-functor. That the quantum graph (143) is f.d. quantum isomorphic to $X$ was shown in Lemma 5.16, so the 2-functor is well-defined on objects. Well-definition on 1-morphisms and 2-morphisms follows from Lemma 5.17. Compositionality is clear by comparing the composition of quantum graph isomorphisms and intertwiners [14, Def. 3.18] to that of UPTs and modifications. Essential surjectivity on objects follows immediately from the last statement of Lemma 5.16. That the equivalence is in fact an isomorphism on Hom-categories follows from Lemma 5.17.

Remark 5.21. By Theorem 5.20, we recover previous results about quantum graphs and finitedimensional quantum isomorphisms. Indeed, one may immediately apply Theorem 4.14 to obtain a classification of finite-dimensional quantum graph isomorphisms in terms of simple Frobenius monoids in the category of finite-dimensional $*$-representations of the quantum automorphism group algebra. This classification has already been shown in [15]. By Theorem 3.14, we also obtain a correspondence between quantum isomorphisms from a quantum graph and $*$-representations of Hopf-Galois objects for its quantum automorphism group, which has already been shown in [5].

Theorem 5.20 additionally shows us that a finite-dimensional isomorphism from a quantum graph extends to a unitary pseudonatural transformation of fibre functors on the whole category of continuous unitary representations of the quantum automorphism group of the quantum graph. These transformations can be interpreted physically; this will be discussed in future work. This is a step towards finding an operational interpretation of quantum isomorphisms between quantum graphs, generalising the interpretation of quantum isomorphisms between classical graphs in terms of nonlocal games [1]. 


\section{References}

[1] Albert Atserias, Laura Mančinska, David E Roberson, Robert Šámal, Simone Severini, and Antonios Varvitsiotis. Quantum and non-signalling graph isomorphisms. Journal of Combinatorial Theory, Series B, 136:289-328, 2019. arXiv:1611.09837.

[2] Teodor Banica. Symmetries of a generic coaction. Mathematische Annalen, 314(4):763-780, 1999. arXiv:math/9811060.

[3] Julien Bichon. Galois extension for a compact quantum group. 1999. arXiv:math/9902031.

[4] Julien Bichon. Hopf-Galois objects and cogroupoids. Rev. Un. Mat. Argentina, 55(2):11-69, 2014. arXiv: 1006.3014.

[5] Michael Brannan, Alexandru Chirvasitu, Kari Eifler, Samuel Harris, Vern Paulsen, Xiaoyu Su, and Mateusz Wasilewski. Bigalois extensions and the graph isomorphism game. Communications in Mathematical Physics, pages 1-33, 2019. arXiv:1812.11474.

[6] Runyao Duan, Simone Severini, and Andreas Winter. Zero-error communication via quantum channels, noncommutative graphs, and a quantum Lovász number. IEEE Transactions on Information Theory, 59(2):1164-1174, 2012. arXiv:1002.2514.

[7] Chris Heunen and Martti Karvonen. Monads on dagger categories. Theory and Applications of Categories, 31(35):1016-1043, 2016. arXiv:1602.04324.

[8] Chris Heunen and Jamie Vicary. Categories for Quantum Theory: An Introduction. Oxford Graduate Texts in Mathematics Series. Oxford University Press, 2019. doi:10.1093/oso/ 9780198739623.001 .0001$.

[9] André Joyal and Ross Street. An introduction to Tannaka duality and quantum groups. In Category theory, pages 413-492. Springer, 1991. URL: http://web.science.mq.edu.au/ street/ CT90Como.pdf.

[10] Tom Leinster. Basic bicategories. 1998. arXiv:math/9810017.

[11] Saunders MacLane. Natural associativity and commutativity. Rice Institute Pamphlet-Rice University Studies, 49(4), 1963.

[12] Saunders MacLane. Categories for the working mathematician, volume 5. Springer Science \& Business Media, 2013.

[13] Paul-André Melliès. Functorial boxes in string diagrams. In International Workshop on Computer Science Logic, pages 1-30. Springer, 2006. URL: https://www.irif.fr/ mellies/mpri/ mpri-ens/articles/mellies-functorial-boxes.pdf.

[14] Benjamin Musto, David Reutter, and Dominic Verdon. A compositional approach to quantum functions. Journal of Mathematical Physics, 59(8):081706, 2018. arXiv:1711.07945.

[15] Benjamin Musto, David Reutter, and Dominic Verdon. The Morita theory of quantum graph isomorphisms. Communications in Mathematical Physics, 365(2):797-845, 2019. arXiv:1801. 09705.

[16] Sergey Neshveyev and Lars Tuset. Compact Quantum Groups and Their Representation Categories. Collection SMF.: Cours spécialisés. Société Mathématique de France, 2013.

[17] Peter Selinger. A survey of graphical languages for monoidal categories. In New Structures for Physics, pages 289-355. Springer, 2010. arXiv:0908.3347. 
[18] Dominic Verdon. Entanglement equivalences of covariant channels. 2020. arXiv:2012.05761.

[19] Dominic Verdon. Unitary pseudonatural transformations. 2020. arXiv:2004.12760.

[20] Stanisław L. Woronowicz. Tannaka-Krein duality for compact matrix pseudogroups. twisted $\mathrm{SU}(\mathrm{N})$ groups. Inventiones mathematicae, 93(1):35-76, 1988. URL: http://resolver.sub. uni-goettingen.de/purl?PPN356556735_0093.

\section{Appendix 1: Rigid $C^{*}$-tensor categories are pivotal dagger categories}

Here we complete the proof of Theorem 2.23, which states that a $C^{*}$-tensor category equipped with standard solutions to the conjugacy equations is a pivotal dagger category. We first recall the relevant results from $[16]$.

Definition 6.1 ([16, Theorem 2.2.16]). Let $\mathcal{C}$ be a $C^{*}$-tensor category with conjugates and let $X$ be some object. Then a standard solution of the conjugate equations for $X$ is a choice of $\left[X^{*}, R, \bar{R}\right]$ as in Definition 2.22 such that

$$
R^{\dagger}\left(\operatorname{id}_{X^{*}} \otimes f\right) R=\bar{R}^{\dagger}\left(f \otimes \operatorname{id}_{X^{*}}\right) \bar{R}
$$

for every morphism $f: X \rightarrow X$.

Proposition 6.2 ([16, Proposition 2.2.15]). Let $\left[X^{*}, R, \bar{R}\right]$ and $\left[X^{* \prime}, R^{\prime}, \bar{R}^{\prime}\right]$ be two standard solutions of the conjugate equations for an object $X$. Then there exists a unitary $T: X^{*} \rightarrow X^{* \prime}$ such that $R^{\prime}=\left(T \otimes \operatorname{id}_{R}\right) R$ and $\bar{R}^{\prime}=\left(\operatorname{id}_{R} \otimes T\right) \bar{R}$.

Lemma 6.3 ([16, Proof of Theorem 2.2.18]). Let $\left(R_{X}, \bar{R}_{X}\right)$ be a standard solution of the conjugate equations for $X$, and $\left(R_{Y}, \bar{R}_{Y}\right)$ be a standard solution of the conjugate equations for $Y$. Then the following choice of $R$ and $\bar{R}$ are a standard solution of the conjugate equations for $X \otimes Y$ :

$$
R=\left(\operatorname{id}_{Y^{*}} \otimes R_{X} \otimes \operatorname{id}_{Y}\right) R_{Y} \quad \bar{R}=\left(\operatorname{id}_{X} \otimes \bar{R}_{Y} \otimes \operatorname{id}_{X^{*}}\right) \bar{R}_{X}
$$

The following additional result is clear.

Lemma 6.4. The morphisms $R_{\mathbb{1}}:=\mathrm{id}_{\mathbb{1}}: \mathbb{1} \rightarrow \mathbb{1} \otimes \mathbb{1}$ and $\bar{R}_{\mathbb{1}}:=\mathrm{id}_{\mathbb{1}}: \mathbb{1} \rightarrow \mathbb{1} \otimes \mathbb{1}$ are standard solutions to the conjugacy equations for the object $\mathbb{1}$.

In a $C^{*}$-tensor category $\mathcal{C}$ where standard solutions to the conjugate equations have been chosen, one may consider the usual contravariant right duals functor.

Theorem 6.5 ([16, Theorem 2.2.21]). Where standard solutions to the conjugate equations have been chosen, the right duals functor is unitary and its square is naturally isomorphic to the identity functor.

Proof. The definition of the natural isomorphism is important for our considerations. For any object $X$, both $\left(\bar{R}_{X}, R_{X}\right)$ and $\left(R_{X^{*}}, \bar{R}_{X^{*}}\right)$ are standard solutions to the conjugate equations for $X^{*}$. Therefore, by Proposition 6.2 , we obtain a unitary $\iota_{X}: X^{* *} \rightarrow X$ such that:

$$
\bar{R}_{X}=\left(\iota_{X} \otimes \operatorname{id}_{X^{*}}\right) R_{X^{*}} \quad R_{X}^{\dagger}=\bar{R}_{X^{*}}^{\dagger}\left(\operatorname{id}_{X^{*}} \otimes \iota_{X}^{\dagger}\right)
$$

These unitaries $\left\{\iota_{X}\right\}$ are the components of the natural isomorphism from the double duals functor to the identity functor. 
It remains only to show that the unitary natural isomorphism $\iota$ is monoidal. The double duals functor may be given a monoidal structure as follows. First we observe that, since (147) are a standard solution to the conjugacy equations for $X \otimes Y$, by Proposition 6.2 there exists a unitary $\mu_{X, Y}: Y^{*} \otimes X^{*} \rightarrow$ $(X \otimes Y)^{*}$ such that:
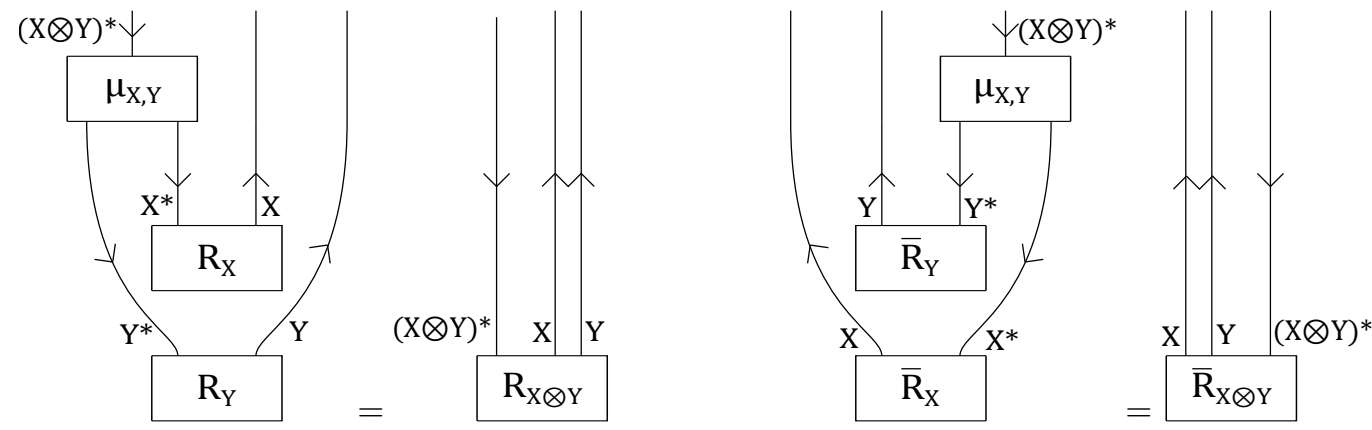

We then define the multiplicators of the double duals functor as follows, for any pair of objects $X, Y$ of $\mathcal{C}$ (using a double upwards arrow to denote a double dual object):

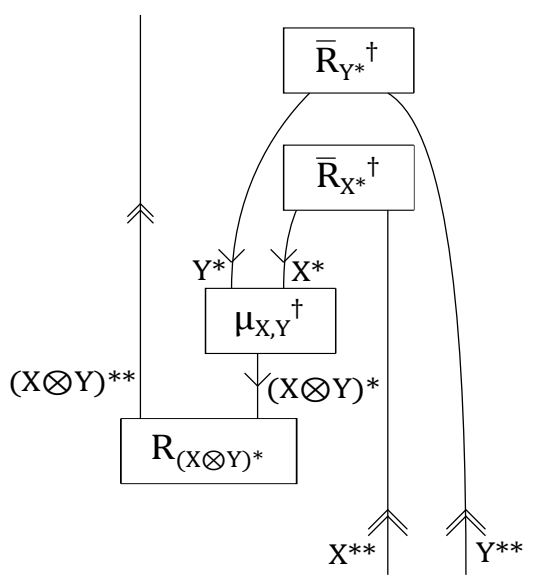

We also observe that, by Lemma 6.4 and Proposition 6.2, there exists a unitary $\nu: \mathbb{1}^{*} \rightarrow \mathbb{1}$ such that:

$$
\nu \circ R_{\mathbb{1}}=\mathrm{id}_{\mathbb{1}} \quad \nu \circ \bar{R}_{\mathbb{1}}=\mathrm{id}_{\mathbb{1}}
$$

This simply implies that $\nu^{\dagger}=R_{\mathbb{1}}=\bar{R}_{\mathbb{1}}$. We then define the unitor of the double duals functor as

$$
\left(\operatorname{id}_{\mathbb{1}^{* *}} \otimes R_{\mathbb{1}}^{\dagger}\right) R_{\mathbb{1}^{*}} .
$$

It is straightforward to check that the unitor and multiplicators just defined are a unitary monoidal structure for the double duals functor.

We also have the following lemma.

Lemma 6.6. For every object $X$ we have equalities

$$
\iota_{X}=\left(\mathrm{id}_{X} \otimes \bar{R}_{X^{*}}^{\dagger}\right)\left(\bar{R}_{X} \otimes \mathrm{id}_{X^{* *}}\right) \quad \iota_{X}^{\dagger}=\left(\operatorname{id}_{X^{* *}} \otimes R_{X}^{\dagger}\right)\left(R_{X^{*}} \otimes \mathrm{id}_{X}\right)
$$

Proof. For the first equality:

$$
\begin{aligned}
\iota_{X}=\left(\iota_{X} \otimes R_{X}^{\dagger} \otimes \bar{R}_{X^{*}}^{\dagger}\right)\left(R_{X^{*}} \otimes \bar{R}_{X} \otimes \operatorname{id}_{X^{* *}}\right) & =\left(\operatorname{id}_{X} \otimes R_{X}^{\dagger} \otimes \bar{R}_{X^{*}}^{\dagger}\right)\left(\bar{R}_{X} \otimes \bar{R}_{X} \otimes \operatorname{id}_{X^{* *}}\right) \\
& =\left(\operatorname{id}_{X} \otimes \bar{R}_{X^{*}}^{\dagger}\right)\left(\bar{R}_{X} \otimes \operatorname{id}_{X^{* *}}\right)
\end{aligned}
$$

The second equality is shown similarly. 
Proposition 6.7. The unitary natural isomorphism of Theorem 6.5 is monoidal.

Proof. Compatibility with the multiplicators is seen as follows:
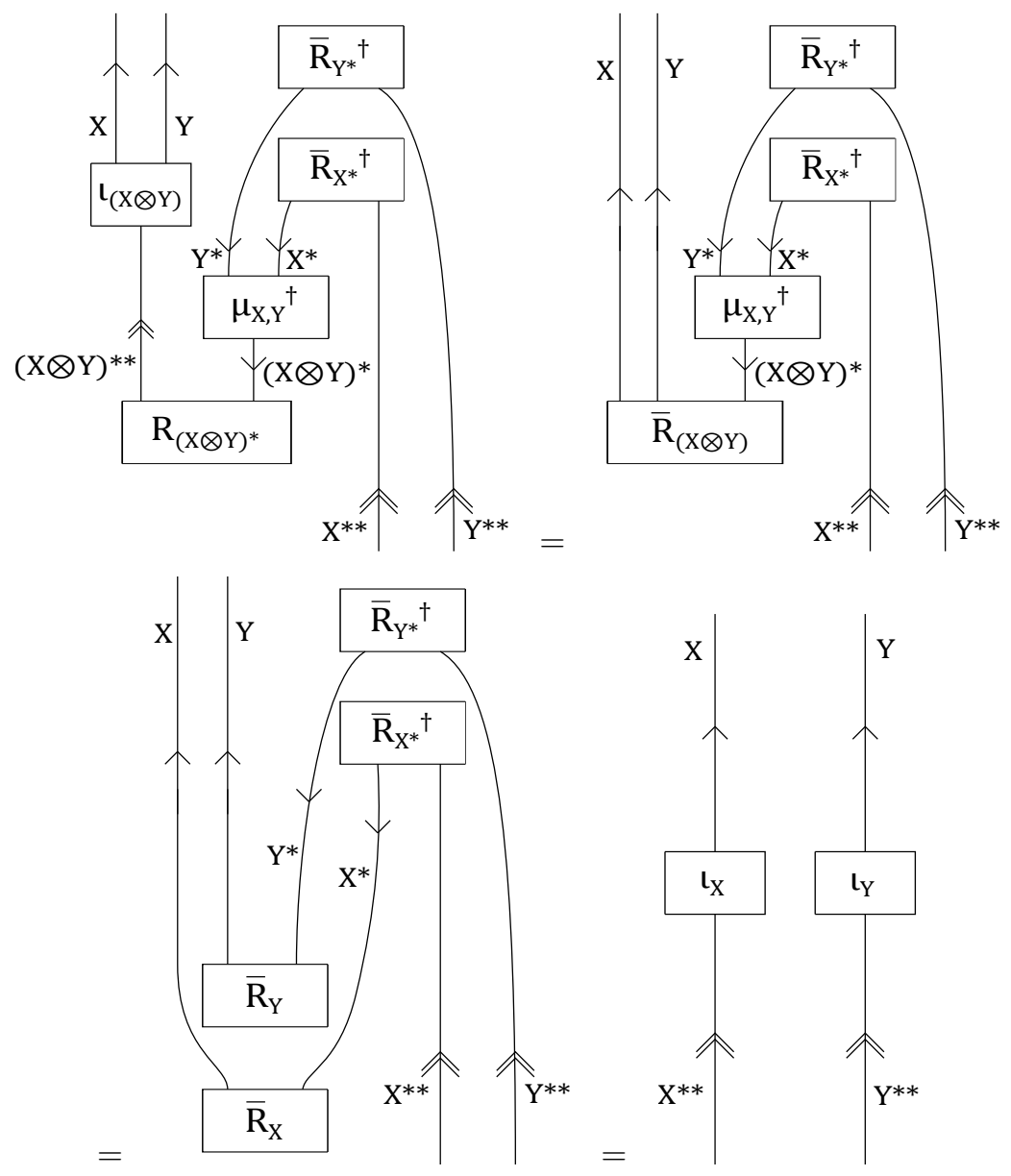

Here the first equality is by (148); the second equality is by (149); and the third equality is by Lemma 6.6.

For compatibility with the unitor we observe that, by Lemma 6.6 , the unitor is precisely $\iota_{\mathbb{1}}^{\dagger}$. But then postcomposing by $\iota_{\mathbb{1}}$ we have $\iota_{\mathbb{1}} \iota_{\mathbb{1}}^{\dagger}=\mathrm{id} \mathbb{1}$, as desired.

The proof that a rigid $C^{*}$-tensor category equipped with standard solutions to the conjugacy equations is a pivotal dagger category is therefore complete. 